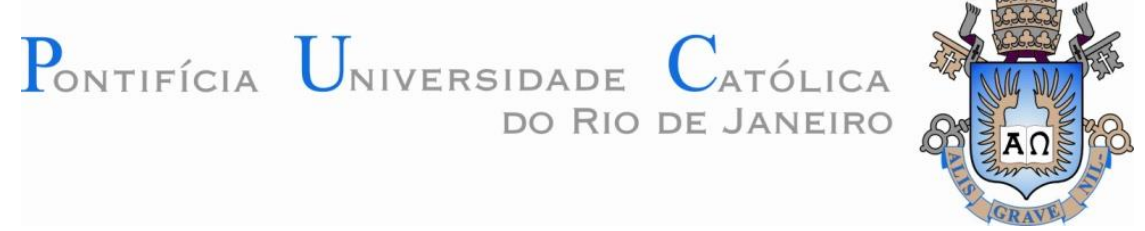

lam Palatnik de Sousa

\title{
Metrological Reliability of Transcranial Magnetic Stimulation
}

Dissertação de Mestrado

Dissertation presented to the Programa de PósGraduação em Metrologia (Área de concentração: Metrologia para Qualidade e Inovação), PUC-Rio as partial fulfillment of the requirements for the degree of Mestre em Metrologia.

Advisor: Prof ${ }^{\mathrm{a}}$. Elisabeth Costa Monteiro Co-Advisor: Prof. Carlos Roberto Hall Barbosa

Rio de Janeiro

April 2016 


\section{Pontifícia Universidade Católica $_{\text {a }}$

lam Palatnik de Sousa

\section{Metrological Reliability of Transcranial Magnetic Stimulation}

Dissertation presented to the Programa de Pós-Graduação em Metrologia da PUC-Rio as partial fulfillment of the requirements for the degree of Mestre em Metrologia. Approved by the following commission:

Prof ${ }^{\mathrm{a}}$. Elisabeth Costa Monteiro

Advisor

Programa de Pós-Graduação em Metrologia - PUC-Rio

Prof. Carlos Roberto Hall Barbosa

Co-Advisor

Programa de Pós-Graduação em Metrologia - PUC-Rio

Prof. Marcio Nogueira Souza

Universidade Federal do Rio de Janeiro - UFRJ

Prof. Sergio Eduardo de Carvalho Machado

Instituto de Psiquiatria - UFRJ

Prof. Eduardo Costa da Silva Departamento de Eng. Elétrica - PUC-Rio

Prof. Márcio da Silveira Carvalho

Coordenador Setorial de Pós-Graduação do Centro Técnico Científico -

PUC-Rio

Rio de Janeiro, April $1^{\text {st }}, 2016$ 
All rights reserved.

\section{lam Palatnik de Sousa}

Graduated as a Bachelor in Physics from the Federal University of Rio de Janeiro (Universidade Federal do Rio de Janeiro - UFRJ).

Bibliographic Data

Palatnik de Sousa, lam

Metrological Reliability of Transcranial Magnetic Stimulation / lam Palatnik de Sousa ; advisor: Elisabeth Costa Monteiro ; co-advisor: Carlos Roberto Hall Barbosa. - 2016.

100 f. : il. color. ; $30 \mathrm{~cm}$

Dissertação (mestrado)-Pontifícia Universidade Católica do Rio de Janeiro, Centro Técnico Científico, Programa de Pós-Graduação em Metrologia (Área de concentração: Metrologia para Qualidade e Inovação), 2016.

Inclui bibliografia

1. Metrologia - Teses. 2. Estimulação magnética transcraniana. 3. Segurança. 4. Confiabilidade metrológica. 5. Distância segura. I. Monteiro, Elisabeth Costa. II. Barbosa, Carlos Roberto Hall. III. Pontifícia Universidade Católica do Rio de Janeiro. Centro Técnico Científico. Programa de Pós-Graduação em Metrologia. IV. Título.

CDD: 389.1 
To my grandfather, Marcos Palatnik, the first scientist in our family. To my cousin, Ezequiel Leon Saidman, the newest scientist in our family. 


\section{Acknowledgements}

I am very grateful for the support and incentive that my family has provided since a very young age. There is a deep sense of respect for knowledge that made them role models I've always admired. Thank you, Clarisa, Elisabeth, Marcos and Olga.

I've also been fortuned to have supervisors that believed in me, giving me space to develop my ideas and grow as researcher. As such, I thank Mohammed el Massalami, Rafael Linden, Elisabeth Costa Monteiro and Carlos Roberto Hall Barbosa. I also thank countless professors I've had, too many to list by name here, that always inspired me to ask questions.

There are many friends that I want to thank, even if I might lack the proper words to do so. Gabriel, for always one-upping me on the knowledge of random topics, and for the most philosophically intense walks registered on the recent century. Felipe, for the many moments of laughter, and for the moments without laughter too. Elaine, for the epic conquests on Age of Empires, and for constantly reminding me of the honest drive of those who pursue knowledge. Luane, "maninha", for years of believing in each step I take.

I'm also grateful to the members of the Irish sessions of Rio de Janeiro, for providing me with an environment of great acceptance where I learned not just about the traditions of Irish music, but also to not be afraid of making mistakes when in front of others. Thank you for reminding me each month of the key role of music in life.

I thank Professor Sérgio Machado, Daniel Contarini and the Laboratory of Panic and Respiration in UFRJ for their contribution and generosity; Professor Eduardo Costa da Silva, Professor Luis Gusmão and the Nucleus of Electronic Instrumentation and Sensors (Núcleo de Instrumentação Eletrônica e Sensores NIEIS) in PUC Rio for their immense aid in the design of the transducer; and the funding by the National Council for Scientific and Technologic Development (Conselho Nacional de Desenvolvimento Científico e Tecnológico - CNPq). 


\section{Abstract}

Palatnik de Sousa, Iam; Costa Monteiro, Elisabeth (Advisor) ; Hall Barbosa, Carlos (Co-Advisor). Metrological Reliability of Transcranial Magnetic Stimulation. Rio de Janeiro, 2016. 100p. MSc. Dissertation - Programa de Pós-Graduação em Metrologia (Área de concentração: Metrologia para Qualidade e Inovação), Pontifícia Universidade Católica do Rio de Janeiro.

A study of the current status of the metrological reliability of Transcranial Magnetic Stimulation (TMS) is presented. The matter of safety is approached in three major aspects: The safety and performance of the TMS devices; the safety regarding exposure limits for patients, staff and the general public; and the safety of the therapeutic protocol and of the treatment parameters. Proposals for a harmonized reporting framework and the basis for a possible future TMS safety and performance technical standard are also presented. The results of simulations and measurements of the magnetic flux densities emitted by two brands of TMS devices are reported, with the corresponding calculations for the safe distances regarding staff exposure, using the methods promulgated by the guidelines of the International Commission on Non Ionizing Radiation Protection (ICNIRP). These distances are compared to the previous estimates found in literature. The development of both the simulation routines and the measurement system are described, including possible future applications in other studies and metrological aspects of measurement uncertainty.

\section{Keywords}

Transcranial Magnetic Stimulation; Safety; Metrological Reliability; Safe Distance 


\section{Resumo}

Palatnik de Sousa, Iam; Costa Monteiro, Elisabeth; Hall Barbosa, Carlos. Confiabilidade Metrológica da Estimulação magnética Transcraniana. Rio de Janeiro, 2016. 100p. Dissertação de Mestrado - Programa de PósGraduação em Metrologia (Área de concentração: Metrologia para Qualidade e Inovação), Pontifícia Universidade Católica do Rio de Janeiro.

Um estudo do atual estado da confiabilidade metrológica da Estimulação Magnética Transcraniana (TMS) é apresentado. A questão da segurança é abordada em três aspectos principais: A segurança e desempenho dos equipamentos de TMS; a segurança em relação aos limites de exposição para operadores do equipamento e pacientes; e a segurança do protocolo terapêutico e dos parâmetros de tratamento. Propostas para um protocolo de relatório harmonizado e a base de uma possível futura norma técnica para equipamentos de TMS também são apresentadas. Os resultados de simulações e medições da densidade de fluxo magnético emitido por equipamentos de TMS de duas marcas são relatados, com os cálculos correspondentes das distâncias seguras em relação a exposição de operadores do equipamento, usando os métodos promulgados pelas diretrizes da Comissão Internacional de Proteção Contra a Radiação Não Ionizante (ICRNIP). Estas distâncias são então comparadas com estimativas prévias encontradas na literatura. $\mathrm{O}$ desenvolvimento das rotinas de simulação e do sistema de medição é descrito, incluindo possíveis futuras aplicações em outros estudos e aspectos metrológicos de incerteza de medição.

\section{Palavras-chave}

Estimulação Magnética Transcraniana; Segurança; Confiabilidade Metrológica; Distância Segura. 


\section{Summary}

1. Introduction 16

1.1. Objective 18

1.1.1. Unification of terminology and conformity assessment $\quad 18$

1.1.2. Development of a measuring system 19

1.1.3. Development of a TMS simulation 19

1.1.4. Estimation of the Safe distance 19

2 Transcranial Magnetic Stimulation 21

2.1. Overview of the technique 21

2.2. The Neuro-MS/D device and software 27

2.3. Example of stimulation protocol 28

2.3.1. Depressions 28

2.3.2. Auditory Hallucinations 29

2.3.3. Neurosurgical planning 29

2.4. Device brands and coil models 30

3 Metrological Reliability 32

3.1. Safety and performance of TMS devices 33

3.1.1. Bureau International des Poids et Mesures (BIPM) 33

3.1.2. INMETRO 33

3.1.3. OIML 34

3.1.4. Standardizing Organizations 34

3.1.5. Approval by health surveillance agencies 37

3.2. Exposure limits 38

3.2.1. WHO 38

3.2.2. ICNIRP 38

3.3. Therapeutic protocol 40

3.3.1. CFM 40

4 Harmonization of reporting framework and conformity assessment 41 
4.1. Reporting Framework 41

4.1.1. Safe Protocols 41

4.1.2. Treatment Terminology 43

4.1.3. Treatment Parameters 44

4.1.4. Framework Harmonization Results $\quad 45$

4.2. Conformity assessment 49

4.2.1. Suggestion of a harmonized TMS technical standard 49

4.2.2. Conformity assessment harmonization results 49

5 Simulation 54

5.1. Development of TMS magnetic flux density simulation models 55

5.1.1. Simulation model for the MCB-70 coil 55

5.1.2. Simulation model for the AFEC-02-100-C Coil 58

5.2. Simulation results $\quad 59$

5.2.1. Simulation results for the MCB-70 59

5.2.2. Simulation results for the AFEC-02-100-C 64

6 Measurements $\quad 68$

6.1. Development of a measuring system 68

6.2. Magnetic Transducer design 68

$\begin{array}{ll}\text { 6.3. Measurement results } & 70\end{array}$

6.3.1. Single channel measurements $\quad 70$

$\begin{array}{ll}\text { 6.3.2. Multi channel measurements } & 74\end{array}$

7 Discussion and conclusion $\quad 79$

7.1. Harmonization of standards and terminology 79

7.2. Development of a TMS simulation 80

7.3. Development of a measuring system 82

$\begin{array}{ll}\text { 7.4. Conclusion } & 84\end{array}$

8 Bibliographical references $\quad 85$

9 Annexes $\quad 89$ 


\section{List of Figures}

Figure 1 - Definition of typical TMS stimulation waveform parameters. This example consists of a train of 4 rectangular pulses in a repetitive TMS protocol.

Figure 2 - Neurosoft Neuro-MS/D device, as seen on the equipment brochure [19]. Operator holding a figure-of-8 stimulation coil on the left side, with patient sitting.

Stimulator with three units mounted on a rack on the right side.

The main unit is on top, the cooling unit on the center, and the extra power supply unit on the bottom.

Figure 3 - Diagram of the front panel of the Neuro-MS main unit:

1) connector of coil "Coil supply"; 2) connector

of coil control "Coil control"; 3 ) button of stimulus delivery ("Start" button); 4) indicator of stimulation readiness "Ready"; 5) indicator of coil temperature "Coil temperature"; 6 ) indicator of "Delay". If it glows, it implies that the indicator "Stimulation parameters" displays the delay for capacitor charge switch on in single pulse stimulation mode; 7) indicator of stimulation parameters (indicator "Stimulation parameters"); 8) indicator of units of measurement of specified stimulation parameters " $\mathrm{Hz}$ ", "s" and "min.";

9) indicator of amplitude "Amplitude"; 10) indicator of power supply "Power";

11) enable/disable button for magnetic stimulator ("Power" button);

12) knob of amplitude setting "Amplitude";

13) button with highlight switching on the setting mode of repetitive stimulation session duration "Session";

14) button with highlight switching on the setting mode of repetitive stimulation pause duration "Pause";

15) knob of stimulation parameters setting 
(knob "Stimulation parameters");

16) button with highlight switching on the setting mode of repetitive stimulation train duration "Train";

17) button with highlight switching on the frequency setting of repetitive stimulation "Fre-quency"; 18) button with highlight switching on the single pulse mode "Single pulse".

Figure 4 - External view of the AFEC-02-100-C coil.

Figure 5 - ICNIRP exposure limits comparison. The limits

defined in 2010 are in blue, and the limits defined in 1998 are in red. Occupational limits are shown by thick lines and general public limits in thin lines.

Figure 6 - Naming convention for the windings that form the simulated emitting coils. The axes are also defined as shown in the image.

Figure 7 - Dependence of magnitude of the magnetic flux density with the y coordinate at a distance of $8.3 \mathrm{~mm}$ below the surface of the coil.

Figure 8 - Spatial distribution of $B_{Z}$ on the x-y plane, $8.3 \mathrm{~mm}$ below the surface of the coil.

Figure 9 - $B$ and $d B / d t$ as a function of the distance on the $z$ axis (operator side of the coil). Red and blue curves and axis refer to $\mathrm{dB} / \mathrm{dt}$ and $B$ values, respectively. Red and blue dotted arrows show the safe distance values obtained by direct comparison of simulation data with the limits of $1 \mathrm{~T} / \mathrm{s}$ and $0.0001 \mathrm{~T}$ provided for $\mathrm{dB} / \mathrm{dt}$ and $B$ by the 2003 and 2010 ICNIRP guidelines, respectively. The black squares indicate the measured $\mathrm{dB} / \mathrm{dt}$ values available in literature [16] and show how the simulation closely approximates these experimental results.

Figure 10 - Comparison between the simulation of magnetic flux density on the Neurosoft coil on a vertical line through Coil $_{B}$ center (blue circles) and data digitized from [20] (red crosses). The digitized data was offset 
by $8 \mathrm{~mm}$ on the negative $Z$ direction. Only the $Z$ component of the magnetic flux density is considered.

Figure 11 - Temporal variation of the voltage levels at the surface of the coil, calculated with 10201 area elements.

Figure 12 - Final magnetic sensor coils design.

Figure 13 - Printed sensor coil board.

Figure 14 - Pulse shape measurement with a single channel, at $250 \mathrm{kS} / \mathrm{s}$, centralized, at $100 \%$ absolute amplitude of the Neuro MS/D system, $1 \mathrm{~Hz}$ pulse repetition frequency, with the emitting coil resting at the top of the sensor coil sheet. Note the cosine-like aspect of the active pulse.

Figure 15 - Sinusoidal fit on the active peak of the pulse.

The adjusted frequency was found to be approximately 2490 $\mathrm{Hz}$ with an adjusted $\mathrm{R}^{2}$ of 0.97 .

Figure 16 - Sinusoidal fit on the active peak of the integrated pulse. The adjusted frequency was found to be approximately $2560 \mathrm{~Hz}$ with an adjusted $R^{2}$ of 0.99 , indicating a strong sinusoidal behavior.

Figure 17 - Fast Fourier transform of the active peak of the measured pulse.

Figure 18 - Multichannel measurement with results from the 15 sensor coils superposed. Measurements were taken during 10 seconds at $15 \mathrm{kS} / \mathrm{s}$ for each sensor coil.

Figure 19 - A pulse measured on the multi channel setup.

The cosine-like aspect is not as clear due to an aliasing effect caused by undersampling. It is clear that the current goes in opposing directions on each winding, generating magnetic flux densities, and thus induced EMFs in different polarities.

Figure 20 - Comparison between the results of measurements made at the surface of the coil (magenta circles) and at 2 $\mathrm{cm}$ from the coil's surface (blue); the data provided by [21] at the coil's surface (red); as well as the simulated data 
from Matlab routine at $0.5 \mathrm{~cm}$ from the $y$ axis (black circles), along the $z$ axis. Only the $Z$ component of the magnetic flux density is considered. 


\section{List of Tables}

Table 1 - Data compiled in [1]. Maximum safe duration in seconds of single trains of rTMS based on the National Institute of Neurological Disorders and Stroke experience. Values marked in red are the longest durations tested. No after discharge or spread of excitation has been encountered with single trains of rTMS at these combinations of stimulus frequency and intensity.

Table 2 - Updated safe parameters compiled in [2].

Safety recommendations for inter-train intervals for 10 trains at $<20 \mathrm{~Hz}$. The maximum duration of pulses for individual rTMS trains at each stimulus intensity should not exceed those listed in the Part B of the table. A consensus has been reached in adopting this table at this point. Values marked in red are the longest durations tested.

Table 3 - Comparison between CFM and Consensus [2] recommendations. CFM values for the pulses in one train are found by dividing the total number of pulses by the total number of trains, and number of treatment days, for the case of depressions. Values related to the maximum duration of pulses taken from tables 4 and 5 from Consensus [2]. Numbers in red are the longest value tested up until the point of the 2008 workshop.

Table 4 - Devices studied in section, with models, brands and corresponding label (TMS $S_{A}$ through $\mathrm{TMS}_{\mathrm{F}}$ ). 


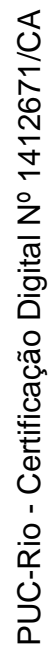

One accurate measurement is worth a thousand expert opinions. - Grace Hopper 


\section{1. Introduction}

Transcranial Magnetic Stimulation (TMS) is a neuromodulation technique based on a rapidly changing current passing through a coil placed above the scalp [1]. The magnetic field generated by the coil allows for electric energy to get across the scalp and skull without the typical side effects of direct percutaneous electrical stimulation [1].

The pulses generated by the device can depolarize neurons and, with repeated application, they can modulate cortical excitability. This has been shown to result in behavioral consequences and has a therapeutic potential [2].

Presently, TMS plays a key role in the treatment of medication resistant major depression [2], among other mental disorders [3,4]. The demonstrated efficacy and lack of major side effects make it a very innocuous and competitive alternative compared to other treatments such as Electroconvulsive Therapy (ECT). This goes in accord with the principles of biometrology [5]. Thus, studying and looking for ways to improve the reliability of this technique become relevant as ways of ensuring safer treatment conditions for both patients and staff.

This technique is currently approved by Health Surveillance Agencies of Australia, Brazil, Canada, European Union, Israel, New Zealand, Russia and United States, among others [2,6,7].

Registration by health agencies requires that the TMS devices demonstrate compliance with various technical standards. Up to the present, however, no particular standard containing specific requirements for TMS devices has been published $[8,9]$. Besides a complete description of the stimulus, aspects regarding device reliability, including safety and performance checks, should be satisfactorily considered [10,5].

Considering that TMS is a technique involving the delivery of a dose of non ionizing radiation, several concerns regarding exposure and associated relevant definitions are raised. Typical TMS treatments use pulse repetition frequencies below $25 \mathrm{~Hz}$ [1], which are often called Extremely Low Frequencies (ELF) [11]. 
The World Health Organization (WHO) has addressed magnetic field safety in several publications [12,13]. Notably, it has encouraged the adoption of exposure limits as defined on the guidelines published by the International Commission on Non-Ionizing Radiation Protection (ICNIRP) [14].

ICNIRP has issued guidelines for protection against magnetic and electromagnetic fields in different occasions [14,15,16,17]. The 1998 guideline presents requirements for a frequency range spanning from $0 \mathrm{~Hz}$ to $300 \mathrm{GHz}$ [14]. In 2003, ICNIRP published an additional document containing information regarding procedures for testing compliance with the 1998 guideline, for nonsinusoidal and pulsed fields [15]. The 2010 and 2014 guidelines revise the recommendations for $1 \mathrm{~Hz}$ to $100 \mathrm{kHz}$ and static fields, respectively [16,17].

The ICNIRP guidelines can be used as a basis to determine which distances would be within occupational safe limits for operators, considering the exposure to the fields emitted by the TMS device $[18,19]$.

In [18], using measurements performed with a calibrated coil and considering the magnetic field exposure limits recommended by the 2003 ICNIRP guideline [15], a distance of about $0.7 \mathrm{~m}$ or more was considered to be safe for the TMS device evaluated, consisting of a MC-B70 coil by Medtronic Synectics with a MegPro unit.

Notably, Karlstrom et al. [18] provided experimental plots showing the pulse shape for the time derivative of the magnetic flux density and for the variation of the maximum value of this quantity as a function of the vertical distance to the coil center.

Later, by means of a phantom study published in 2010, Lu and Ueno identified the safe distance according to 2003 ICNIRP as being $1.1 \mathrm{~m}$ [19]. It must be noted that the two above referred studies did not evaluate the same brand and model of TMS device.

Lu and Ueno [19] have analyzed the dependence of induced currents in a real human model, testing different coil shapes, distances between coil and human model, and rotation of the coil in space. Namely, the coils used included a figureof-eight and a round model, of unspecified brands.

In 2003, Nadeem et al. [20] have used the impedance method to calculate induced currents and electric field distributions in the human brain associated with TMS, using a three-dimensional human head model. The magnetic field simulated 
was generated with a virtual model of a figure-of-eight MC-B70 (Medtronic) TMS coil, calculated using the Biot-Savart's law [20]. The study provides parameters regarding both coil and current shapes.

While TMS has been studied by several groups over the last decades, there are several aspects of safety and performance of TMS devices that could be revisited and further analyzed and enhanced. Notably there is no particular standard designed specifically for this type of medical equipment, the safe distance studies consider older versions of exposure protection guidelines, and there is an abundant variety of non harmonized terminology and concepts that often can be mixed in literature and other types of publications and documents. Different regions also use different standards and terminology to refer to TMS technologies [8,9].

This elicits the need to evaluate the current status of TMS technology, identify all the possible gaps and any room for improvement and, either through simulations or measurements, to provide more data to allow for this advancement. This includes revisiting the safe distance values given in literature in light of the more recently published ICNIRP guidelines [17], and discussing some key aspects of previous studies on the theme while also providing new data for consideration.

\section{1. Objective}

This work aims at evaluating the current status of the metrological reliability of the TMS technique and devices. To achieve this goal, a series of approaches were taken, leading to four specific objectives:

- Unification of terminology and conformity assessment;

- Development of a measuring system;

- Development of a TMS simulation; and

- Estimation of the Safe distance.

These specific objectives are described below.

\subsection{1.}

\section{Unification of terminology and conformity assessment}

Different research groups and interested parties also did not seem to have a unified terminology when referring to TMS dose definition. An objective that rose while gathering this information was to develop a metrologically unified 
framework of concepts and terminology regarding TMS treatments, as well as proposing the basis for the first steps towards unified standard that gathers requirements from multiple regulatory documents used around the world.

\subsection{2.}

Development of a measuring system

To obtain the safe distance value, some form of measurement of the typical TMS fields would be necessary to further validate the simulations. At an early point of the research it became clear that TMS fields in general have temporal derivatives on the order of dozens of kiloteslas per second $[18,20]$. Common magnetometers wouldn't be able to handle this intensity levels and required response times, so developing a measuring system specifically designed for this case became a focus point.

\subsection{3.}

\section{Development of a TMS simulation}

The various features defining the measuring system would have to be decided based on the available literature data. However, there was not enough information regarding the specific TMS model available for use at this study. Thus, it became imperative to generate a simulation of the TMS fields to allow for predictions and determining the details of the measuring system.

Furthermore it was noted that two studies in the literature $[18,20]$ dealt with one of the TMS coil models analyzed in this work (MCB-70), and the developed comparisons with these results could be used in the validation of the simulation routine.

\subsection{4.}

\section{Estimation of the Safe distance}

In order to fully characterize the safety of TMS devices, some research groups tried to develop estimates of safe distances in which the occupational exposure limits defined by ICNIRP guidelines $[14,15]$ were not violated $[18,19]$. Since there are newer guidelines available [16], this dissertation intended to update the safe distance previously estimated.

The final goal is to use the validated simulation and the recently published 2010 ICNIRP guidelines [16] to derive more robust estimates for the safe distance for two brands of TMS devices and coils studied: one by Neurosoft (with the 
AFEC-02-100-C figure-of-8 coil) and one by Medtronic (with the MCB-70 figure-of- 8 coil).

The MCB-70 model is the only one explicitly cited in previous studies by Karlström et al. [18] and Nadeem et al. [20], and those publications provide, respectively, experimental measurements and theoretical simulation data about the magnetic flux densities associated with that coil model. Since these experimental and theoretical results had not been previously compared and reconciled in literature, this presented itself as a good opportunity to test and validate the simulation routine, further providing a three dimensional mapping of the simulated field not done by Nadeem et al.

The Neurosoft device is the only TMS device currently approved by ANVISA, and the AFEC-02-100-C was readily available for measurements. This provided a great opportunity to expand the studies to other brands and coil models and learn about the specificities of each model when it comes to the aspect of safe distances. 


\section{2 \\ Transcranial Magnetic Stimulation}

In this chapter a more detailed description of the TMS technique and mechanisms related to the equipment are provided. Different device brands and coil models are also discussed.

\section{1.}

\section{Overview of the technique}

TMS is a type of neurostimulation mainly based on the principle of induction of electric signals in the brain due to the application of rapidly varying magnetic flux density. This stimulus can happen in numerous forms and have sufficient magnitude to depolarize neurons. When TMS pulses are applied repetitively they can modulate cortical excitability, decreasing or increasing it, depending on the parameters of stimulation, even beyond the duration of the train of stimulation. This technique has behavioral consequences and therapeutic potential.

There are many possible TMS protocols with varying parameters. While TMS is used as a generic term, over time, these protocols have received specific names according to their particularities. Rossi et al. [2] have reviewed these protocols and listed them.

Namely, TMS treatments can be broadly categorized in: single pulse TMS, applying one stimulus at a time; paired pulse TMS, applied in pairs of stimuli separated by a variable interval; and repetitive TMS (rTMS), applied in trains of pulses. Fig. 1 exemplifies some of the typical parameters that define these protocols. 


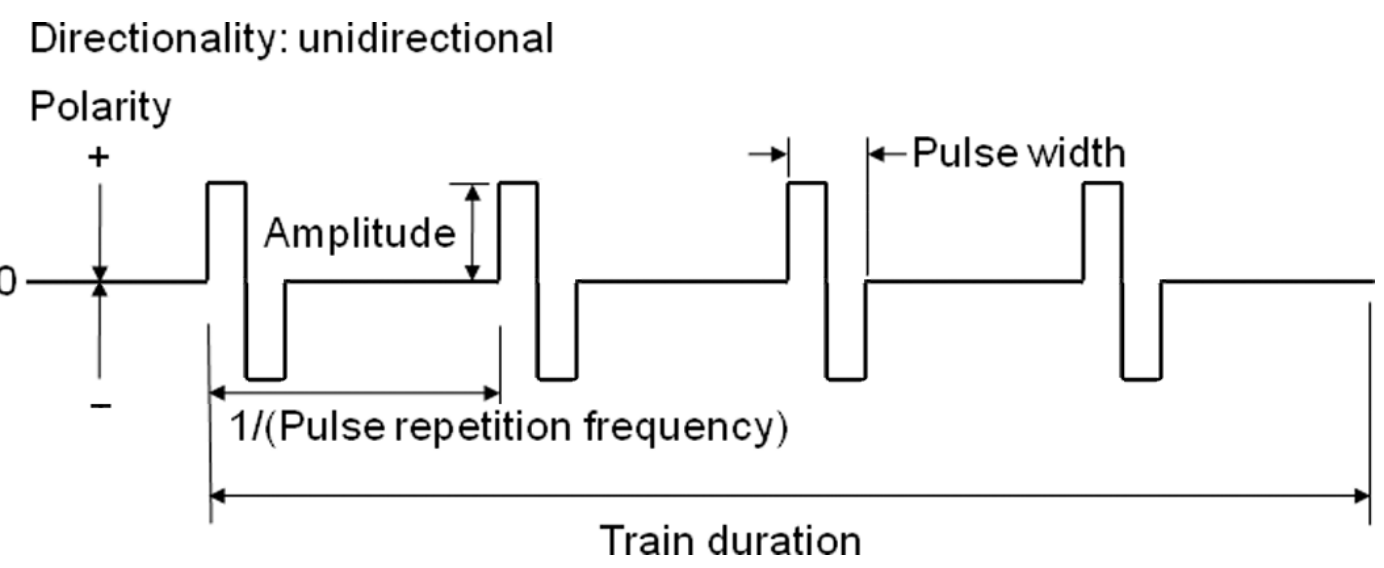

Figure 1 Definition of typical TMS stimulation waveform parameters. This example consists of a train of 4 rectangular symmetrical biphasic pulses in a repetitive TMS protocol.

As for the different uses for each of those protocols, single pulse TMS can be applied for mapping motor cortical outputs, studying central motor conduction time, and studying causal chronometry in brain behavior relations [2].

Paired pulse TMS can be delivered to a single cortical target using the same coil or to two different brain regions using two different coils. Paired pulse techniques can provide measures of intracortical facilitation and inhibition, as well as study cortico-cortical interactions. Pairing can also be done with a peripheral stimulus and a single TMS stimulus, called paired associative stimulation (PAS) $[3,4,21]$.

Repetitive TMS, when multiple stimuli are delivered in trains, can be further classified in conventional and patterned protocols [2]. Conventional rTMS protocols refer to the application of regularly repeated single TMS pulses. The term "fast" or "high-frequency" rTMS refers to stimulus rates higher than $1 \mathrm{~Hz}$, and the term "slow" or "low-frequency" rTMS refers to stimulus rates of $1 \mathrm{~Hz}$ or less. Such a classification is based on the different physiological effects and degrees of risk associated with low and high frequency stimulation [3,4,21].

Patterned rTMS refers to repetitive application of short rTMS bursts at a high inner frequency interleaved by short pauses of no stimulation. Most used to date are the different theta burst (TBS) protocols, in which short bursts of $50 \mathrm{~Hz}$ rTMS are repeated at a rate in the theta range $(5 \mathrm{~Hz})$ as a continuous (cTBS), or intermittent (iTBS) train [2]. 
The device on which the measurements were performed in the present work was the Neuro-MS/D model by Neurosoft, with an AFEC-02-100-C stimulating coil. This device, seen in Fig.2, will be used here as a basic example of the key features and aspects of the stimulation process.

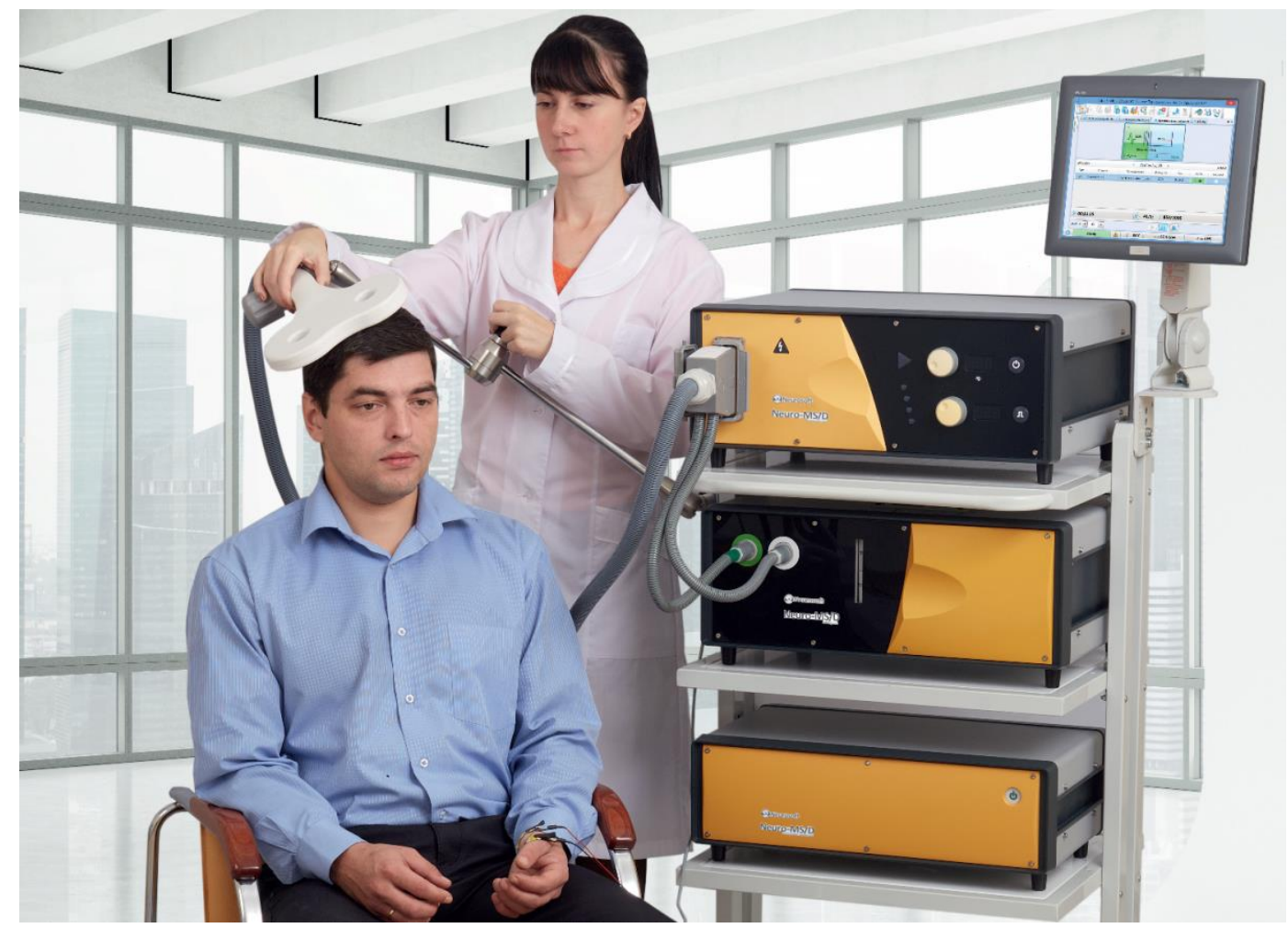

Figure 2. Neurosoft Neuro-MS/D device, as seen on the equipment brochure [22]. The patient is seated and the operator is holding a figure-of -8 stimulation coil on the right side of his scalp. The stimulator has three units mounted on a rack on the right side of the image. The main unit is on top, the cooling unit on the center, and the extra power supply unit on the bottom.

The Neuro-MS/D stimulation system consists of up to three basic units, depending on the desired application. The main unit, seen on fig. 2 at the topmost position of the support rack, is the basis of the device and controls all other units, or can be used as a standalone device.

The cooling unit, seen in the middle portion of the rack in fig. 2, is used for stimulations of longer duration [22]. In those cases the coil could overheat considerably, given the high currents involved in TMS, so the cooling unit pumps cooling liquid through the coil. Alternatively, if this cooling unit is not present, or if the coil is not of a cooling enabled model, the coil has to be allowed to cool down between long stimulation sessions. 
On the bottom portion of the rack seen in fig. 2 is the extra power supply unit. The main unit of the magnetic stimulator is designed to operate with up to $30 \mathrm{~Hz}$ pulse repetition frequency. However, the main unit alone can deliver maximum intensity only at 5 to $7 \mathrm{~Hz}$ frequency. The extra power supply unit makes it possible to increase this frequency up to 20 to $25 \mathrm{~Hz}$, depending on coil type, with a maximum frequency of up to $100 \mathrm{~Hz}$. It is worth reinforcing that both the extra power supply and the cooling units are optional add-ons that give further capabilities to the main unit, which could still work as a stand-alone otherwise.

Besides these units, this system also has a mechanical arm that aids in positioning the coil over the patient's head.

The available stimulation parameters for the main unit, as listed by the device manual are [23]: peak magnetic output of 1.1 to 2.2 tesla (corresponding to $100 \%$ of the device output), depending on the coil type; pulse duration of $250 \mu \mathrm{s}$; maximum frequency at maximum power of $5 \mathrm{~Hz}$; stimulation train duration of 0.5 to $10 \mathrm{~s}$; pauses between trains of 0.5 to $30 \mathrm{~s}$; session time of 0.5 to $30 \mathrm{~min}$; delay for the capacitor charge switch on of up to $9.9 \mathrm{~s}$; minimal number of stimuli before coil overheating at $25{ }^{\circ} \mathrm{C}$ initial temperature, maximum power and $1 \mathrm{~Hz}$ frequency of not less than 500 for the "big ring" coil, not less than 185 for the "small ring coil", and not less than 360 for the "figure-of-8 coil".

The front panel, seen in fig. 3, contains digital indicators displaying all adjustable stimulation parameters and the controls to adjust them. The stimulation parameters can be controlled by the operator either through the front panel of the main unit, as seen in fig. 3, or through the Neuro-MS.NET software installed on a computer that may be connected to the main unit through an USB port.

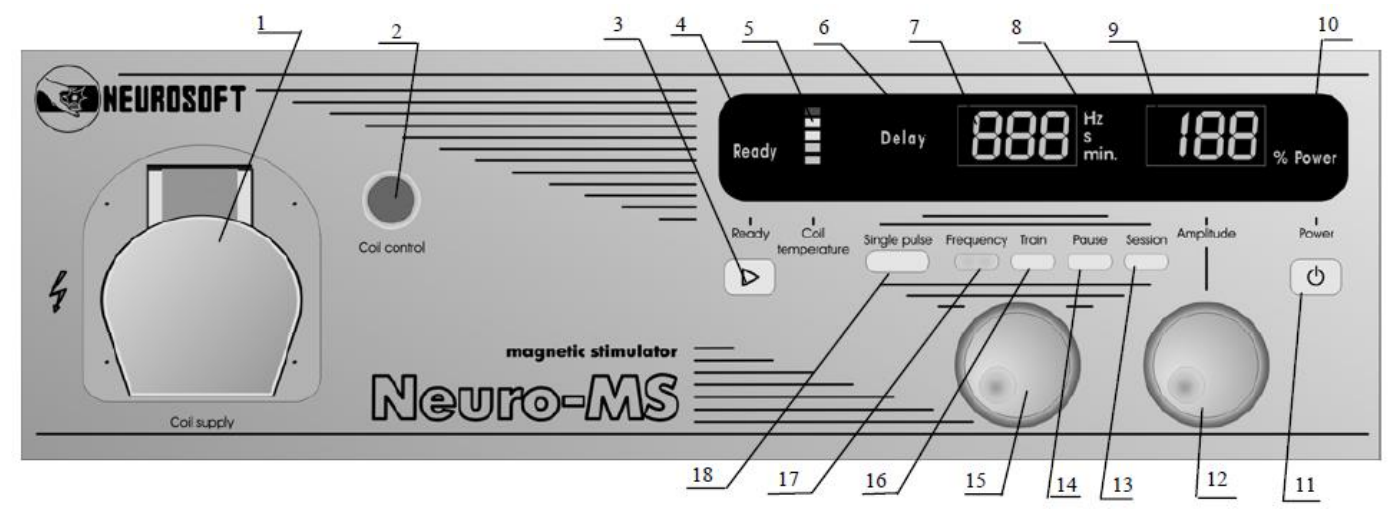

Figure 3. Diagram of the front panel of the Neuro-MS main unit: 1) connector of coil "Coil supply"; 2) connector of coil control "Coil control"; 3) 
button of stimulus delivery ("Start" button); 4) indicator of stimulation readiness "Ready"; 5) indicator of coil temperature "Coil temperature"; 6) indicator of "Delay". If it glows, it implies that the indicator "Stimulation parameters" displays the delay for capacitor charge switch on in single pulse stimulation mode; 7) indicator of stimulation parameters (indicator "Stimulation parameters"); 8) indicator of units of measurement of specified stimulation parameters "Hz", "s" and "min."; 9) indicator of amplitude "Amplitude"; 10) indicator of power supply "Power"; 11) enable/disable button for magnetic stimulator ("Power" button); 12) knob of amplitude setting "Amplitude"; 13) button with highlight switching on the setting mode of repetitive stimulation session duration "Session"; 14) button with highlight switching on the setting mode of repetitive stimulation pause du-ration "Pause"; 15) knob of stimulation parameters setting (knob "Stimulation parameters"); 16) button with highlight switching on the setting mode of repetitive stimulation train duration "Train"; 17) button with highlight switching on the frequency setting of repetitive stimulation "Frequency"; 18) button with highlight switching on the single pulse mode "Single pulse".

It is important to note that, besides defining values for all of these quantities to be applied in a given protocol, the amplitude of the pulses is determined individually for each patient based on a limit defined as the Motor Threshold (MT). Rossi et al. [2] define the resting MT as the minimal intensity required to elicit an electromyogram (EMG) response of at least $50 \mu \mathrm{V}$ with $50 \%$ probability in a fully relaxed muscle. Frequently the muscles used to continuously monitor the EMG are the abductor pollicis brevis or the first dorsal interosseous muscle, on the side contra-lateral to the rTMS application site.

As pointed by systematic reviews made during consensus meetings [1,2], rTMS has been found to be promising as a noninvasive alternative for the treatment of numerous neuropsychiatric conditions, namely: depression, acute mania, bipolar disorders, panic, hallucinations, obsessions/compulsions, schizophrenia, catatonia, post-traumatic stress disorder, drug craving, Parkinson's disease, dystonia, tics, stuttering tinnitus, spasticity, epilepsy, rehabilitation of aphasia or of hand function after stroke, and pain syndromes, such as neuropathic 
pain, visceral pain or migraine. Notably, the largest number of studies seems to be regarding the use of rTMS in the treatment of depression. 


\section{2.}

\section{The Neuro-MS/D device and software}

The TMS device used in this study was a Neuro-MS/D model by Neurosoft. The treatment parameters and routines can be set either manually with controls available on the device or through a software. While all of the parameters displayed in either case can be adjusted, there are further stimulation parameters present in the treatment that can't be changed. The software already has some preset parameter templates that are configured in accord to the limits set by the Brazilian Federal Council of Medicine (Conselho Federal de Medicina-CFM).

Namely, the parameters that can be controlled through the software or device dials are: stimulation type (monophasic, biphasic, burst), absolute amplitude, relative amplitude, pulses frequency in train, pulses in train, number of trains, pause between trains.

Besides those, the position of the stimulation coil relative to the scalp can be varied. This model includes a mechanical arm to aid in this process. The type of stimulation coil may also be changed if multiple models are available, but in this case only the AFEC-02-100-C figure-of-8 coil was used. 


\section{3. \\ Example of stimulation protocol}

The coil manual [24] indicates the angulated figure-of- 8 coils as intended for stimulation of the motor cortex, for cortex focused stimulation after different neurological incidents, such as stroke, and also to study the conduction pathways of visual analysis (occurrence of phosphenes while stimulating gyrus lingualis and cuneus), besides treatment of depressions.

The safe parameters for stimulation recognized by the Brazilian Federal Council of Medicine are presented below for the three different approved and recognized indications [7]: treatment of uni and bipolar depressions, of auditory hallucinations caused by schizophrenia, and for neurosurgical planning depressions. The treatment may only be provided by a physician.

\subsection{1.}

\section{Depressions}

\subsubsection{1.}

a)

Frequency: $10 \mathrm{~Hz}$.

Intensity: $110 \%$ of motor threshold.

Duration of trains: 5 seconds

Number of trains: 25

Interval between trains: 25 seconds

Length of treatment in days: 20 or according to evaluation

Total number of pulses: 25000

Application site: Left dorsolateral prefrontal cortex.

\subsubsection{2.}

b)

Frequency: $5 \mathrm{~Hz}$.

Intensity: $120 \%$ of motor threshold.

Duration of trains: 10 seconds

Number of trains: 25

Interval between trains: 20 seconds

Length of treatment in days: 20 or according to evaluation

Total number of pulses: 25000 
Application site: Left dorsolateral prefrontal cortex.

\subsubsection{3.}

c)

Frequency: $1 \mathrm{~Hz}$.

Intensity: $80 \%$ to $100 \%$ of motor threshold.

Duration of trains: 20 minutes

Number of trains: 1

Interval between trains: Doesn't apply

Length of treatment in days: 20 or according to evaluation

Total number of pulses: 24000

Application site: Right dorsolateral prefrontal cortex.

\subsection{2.}

\section{Auditory Hallucinations}

Frequency: $1 \mathrm{~Hz}$.

Intensity: $80 \%$ to $100 \%$ of motor threshold.

Duration of trains: 20 minutes

Number of trains: 1

Interval between trains: Doesn't apply

Length of treatment in days: 20 or according to evaluation

Total number of pulses: 12000

Application site: Left temporoparietal cortex.

\subsection{3.}

\section{Neurosurgical planning}

Exclusive use in services of excellence, in universities or not, with broad experience in TMS paired with specific systems of neuronavigation. 


\section{4.}

\section{Device brands and coil models}

The Neuro-MS/D model discussed in section 2.1 is just one model of TMS device among numerous brands. Several device models exist, sometime within the same brand, with considerable variations. Most notably, the Medtronic device studied by Karlström et al. [18] and Nadeem et al. [20] has a different pulse frequency when compared to the Neurosoft Neuro-MS/D system. Some older or simpler models from several brands do not have a mechanical arm for positioning the stimulation coil, or may or may not have cooled coils. On the other hand, some of the more modern systems have automated robotic coil positioning, such as models provided by Axilum TMS Robotics.

Even for a specific brand and device model, several types of stimulation coils might be available. While on this study only the AFEC-02-100-C (seen in fig. 4) angulated figure-of- 8 coil was available for analysis, the device brochure lists a total of 10 available coil types, including some cooled variants and some placebo variants for a few of the models. Each coil is listed with the maximum magnetic flux density output at 100\% stimulus amplitude settings with the highest value of $2.9 \mathrm{~T}$ corresponding to the SFEC-02-50 small figure-of- 8 coil, and the lowest value of $0.7 \mathrm{~T}$ corresponding to the DCC-02-125 double cone coil. In general the amplitudes used for stimulation are lower than this absolute maximum, as they are calculated regarding the motor threshold, as described in chapter 1 . 


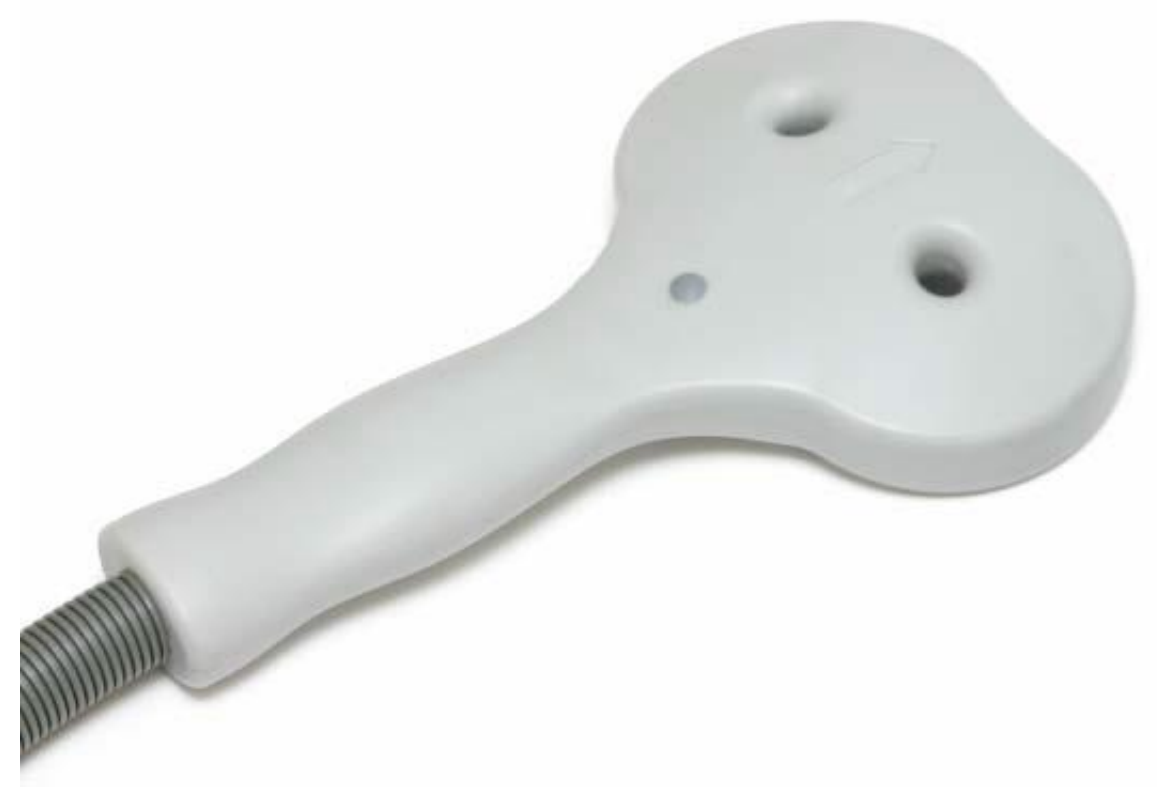

Figure 4 External view of the AFEC-02-100-C coil.

A more detailed technical manual for the coils [24] lists two additional models for the same device, besides the ten included in the brochure. It also clearly shows that the spatial distribution of the magnetic flux densities varies considerably between different coil models, and explains the intended use for each type. 


\section{3 \\ Metrological Reliability}

Medical devices can be assessed according to a number of aspects in regards to their metrological reliability [5,9]. For the case of TMS and in a broad sense, this reliability can be summarized by a key triad of aspects: the safety and performance of the TMS devices; the safety regarding exposure limits for patients, staff and the general public; and the safety of the therapeutic protocol and of the treatment parameters.

The basis of this reliability and of the conformity assessment process is a hierarchy of institutions and regulatory documents, some mandatory, some voluntary. These include National Metrology Institutes (NMI), Health Surveillance Agencies, Standards, Guidelines, Regulations, among others.

In Brazil, an example is the Neuro-MS system [24,23], which is certified by the Instituto Nacional de Metrologia, Qualidade e Tecnologia (Inmetro, the Brazilian NMI) since 2008 and is registered under the number 80342230003 by Agência Nacional de Vigilância Sanitária (ANVISA).

The Inmetro certificate of compliance for this product is available on the Neurosoft website and regards the NBR IEC standards 60601-1:1994 + Emenda:1997, which is a broader and more general standard regarding requirements of basic safety and performance of medical electrical equipment besides the collateral standards 60601-1-2:2006 and 60601-1-4:2004, which cover electromagnetic compatibility and programmable electrical medical systems, respectively which are collateral standards, and 60601-2-10:2002, which is a particular standard for neuromuscular stimulation devices.

Below are defined some of the key Brazilian and international institutions and documents that play a role in the reliability of TMS, directly or indirectly, separated under the first two of aspects in the triad explained on the beginning of this chapter. The third aspect, concerning the therapeutic protocol, is briefly introduced in section 3.3 and more thoroughly explored in chapter 4. 


\section{1. \\ Safety and performance of TMS devices}

\subsection{1. Bureau International des Poids et Mesures (BIPM)}

Established by the Metre Convention on May $20^{\text {th }}, 1875$, the BIPM is an intergovernmental organization, through which Member States act together on matters related to measurement science and measurement standards [25].

The three main roles of the BIPM are based on its impartial and international character, and include: coordinating the realization and improvement of the world wide measurement system to ensure it delivers accurate and comparable measurement results; undertaking selected scientific and technical activities that are more efficiently carried out in its own laboratories in behalf of other Member States; promoting the importance of metrology to science, industry and society.

One among the many stated BIPM objectives [25] is to coordinate international comparisons of national measurement standards through the Consultative Committees of the International Committee for Weights and Measures (CIPM).

As such, the BIPM is a cornerstone of the world wide traceability and reliability of measurement results, and is responsible by the three key publications that serve as a worldwide base for metrology: the International System of Units (SI), the International Vocabulary of Metrology (VIM), and the Guide to the Expression of Uncertainty in Measurement (GUM).

\subsection{2.}

\section{INMETRO}

The National Institute of Metrology, Quality and Technology (Instituto Nacional de Metrologia, Qualidade e Tecnologia - INMETRO) is a federal autarchy that has the mission to provide the Brazilian society with reliability in measurements and products, through Metrology and conformity assessment [26]. It is the Brazilian National Metrology Institute and therefore the central institution of the Brazilian System of Metrology, Quality and Technology. As such, it plays a similar role to the BIPM, in a national capability. 
Notably, this institution is involved in certifying devices regarding their conformity to medical safety and performance standards, either directly or indirectly, through a network of accredited laboratories belonging to the Brazilian System of Conformity Assessment (Sistema Brasileiro de Avaliação da Conformidade - SBAC). As an example, INMETRO certificate ELM-9114 [27] contains information on how the Neuro-MS TMS system by Neurosoft was certified, through the INMETRO licensed Brazilian Institute for Conformity Assessments (Instituto Brasileiro de Ensaios de Conformidade - IBEC).

\subsection{3.}

OIML

The Organization Internationale de Métrologie Légale is an intergovernmental organization founded in 1955 that promotes the global harmonization of legal metrology procedures aiming at establishing the basis for the international trade.

Since its establishment, it has developed a number of guidelines to assist members, particularly developing nations, to draw up appropriate legislation concerning metrology across all facets of society and guidelines on certification and calibration requirements of new products, particularly where such calibration has a legal impact, such as in trade, health care and taxation.

\subsection{4.}

\section{Standardizing Organizations}

Technical standards, published by the International Electrotechnical Commission (IEC) and the International Organization for Standardization (ISO), provide wide-ranging requirements for medical devices that are used by regulators for conformity assessment.

IEC and ISO are two of three global sister organizations (IEC, ISO, ITU) that develop International Standards for the world.

When appropriate, IEC cooperates with ISO (International Organization for Standardization) or ITU (International Telecommunication Union) to ensure that International Standards fit together seamlessly and complement each other. Joint committees ensure that International Standards combine all relevant knowledge of experts working in related areas. 
The IEC 60601 series, promulgated by the IEC, features several aspects pertaining to the basic safety and essential performance for medical electrical (ME) devices. As such, they aim to specify general requirements and to serve as a basis to particular standards [28].

Notably, collateral standards specify general requirements for basic safety and essential performance applicable to a subgroup of ME equipment or to a specific characteristic of all ME equipment not addressed on the more general IEC 60601-1 document. These can be identified by codes using the format 60601-1-X.

Furthermore, particular standards (codes with format 60601-2-X) may modify, replace or delete requirements contained in the IEC 60601-1 standard, as appropriate for a given particular type of ME equipment.

Up to this point, there is no IEC particular standard concerning TMS devices $[8,9]$. However, there are several compliance evaluations in which either IEC standards, or national/regional versions based on those, such as the UL/CSA/EN60601-1, have been explicitly applied.

\subsubsection{1. International Organization for Standardization (ISO)}

ISO is an independent, non-governmental international organization with a membership of 162 national standards bodies. Through its members, it brings together experts to share knowledge and develop voluntary, consensus-based, market relevant International Standards that support innovation and provide solutions to global challenges. [29]

This organ has published the ISO 13485:2003 standard that specifies requirements for a quality management system, where an organization needs to demonstrate its ability to provide medical devices and related services that consistently meet customer requirements and regulatory requirements applicable to medical devices and related services [30]. For instance, the brochure for the MagPro model, by Magventure, states compliance to this standard.

\subsubsection{2. International Electrotechnical Commission (IEC)}

The International Electrotechnical Commission (IEC) is the world leading organization that prepares and publishes International Standards for all electrical, electronic and related technologies. 
Close to 20000 experts from industry, commerce, government, test and research labs, academia and consumer groups participate in IEC Standardization work.

On one hand, according to the IEC 60601-1 standard, collateral standards specify general requirements for basic safety and essential performance applicable to a subgroup of medical electric (ME) equipment and/or a specific characteristic of all ME equipment. On the other hand, particular standards may modify, replace or delete requirements contained on the IEC 60601-1 standard as appropriate for the particular ME equipment under consideration, and may add other basic safety and essential performance requirements.

The particular standard IEC 60601-2-10 relates to particular requirements for the safety and essential performance of nerve and muscle stimulators. This seems like the best approximation for a specific standard that would apply to rTMS devices.

However, the IEC 60601-2-10 standard, under clause 1.1, section 1, states that, among others, equipment destined for brain stimulation (e.g. electroconvulsive therapy equipment) and equipment destined for neurological research should specifically be excluded from that standard's fields of application. It is worth noting that rTMS devices fall under this category.

This denotes the lack of a specific standard for the brain stimulation equipment, and most notably for TMS equipment, since the operation principles of magnetic stimulators that induce currents on nerves, and stimulators that directly deliver currents via electrodes, are quite different and require different safety prescriptions. For example, reference [18] mentions the hazards of staff exposure to doses that surpass the values recommended by [14], [15] and [31] at distances of about less than $70 \mathrm{~cm}$ from the coil surface.

\subsubsection{3.} ABNT

The Brazilian Association of Technical Standards (Associação Brasileira de Normas Técnicas - ABNT) is a private non-profit organization that acts as the national forum of standardization since its inception. It is a founding member of the ISO and also a member of the IEC since it was created. 
It is the institution responsible for the publication of Brazilian Standards (ABNT NBR), and has the mission to provide the Brazilian society with a systematic knowledge through regulatory documents, promoting scientific and technologic development, protecting the environment and defending the consumer.

Notably, it is also responsible for adapting the IEC 60601-1 family to the Brazilian NBR IEC 60601-1 family, which plays a key role in the conformity assessment of medical equipment. In the case of TMS devices, the required standard is the NBR IEC 60601-2-10.

\subsection{5.}

\section{Approval by health surveillance agencies}

TMS is currently approved by Health Surveillance Agencies of Israel, Canada, New Zealand, Brazil, Australia, United States, European Union, among others $[2,6,7]$.

Notably, in the USA, repetitive TMS was cleared in 2008 as a treatment to alleviate symptoms of mildly treatment-resistant depression, in which patients have not found relief from antidepressant medication. In Brazil, in 2012, the Federal Council of Medicine recognized TMS for the treatment of major depression, auditory hallucinations caused by schizophrenia and for neurosurgical planning [7].

The National Health Surveillance Agency of Brazil (Associação Nacional de Vigilância Sanitária - ANVISA) is a regulatory institution that acts on all matters concerning products and services that may affect the Brazilian population's health [32].

Through its Resolution 27 of June $21^{\text {st }}, 2011$ [33], ANVISA has defined the procedures for the compulsory certification of equipments under health surveillance regime. This document, in turn, makes certification by the SBAC compulsory, for these equipments to prove compliance to Resolution 56 of April $6^{\text {th }}$ of 2001 [34], which establishes broad safety and performance requirements applicable to health products. 


\section{2. \\ Exposure limits}

\subsection{1. WHO}

The World Health Organization is a specialized part of the United Nations concerned with international public health. In 1973, WHO initiated the Health Criteria Programme, with the objectives of: assessing information on the relationship between exposure to environmental pollutants and human health, and to provide guidelines for setting exposure limits; identifying new or potential pollutants; identifying gaps in knowledge concerning the health effects of pollutants; and promoting the harmonization of toxicological and epidemiological methods in order to have internationally comparable results [35].

Since then, WHO has developed documents regarding electromagnetic field $(\mathrm{EMF})$ safety in different frequency ranges: from Extremely Low Frequency (ELF) fields [11,35] to static fields [36].

Furthermore, WHO has issued a framework for the development of healthbased EMF standards [12]. This document mentions that large disparities between national limits and international guidelines can foster confusion for regulators and policy makers, increase public anxiety and provide a challenge to manufacturers and operators of communications systems who need to tailor their products to each market. These factors have motivated WHO to build a framework for developing health-based EMF exposure standards using a rational scientificallydriven process.

Importantly, this document also mentions that WHO endorses the limits given by the guidelines of ICNIRP.

\subsection{2.}

\section{ICNIRP}

ICNIRP has issued guidelines for protection against magnetic and electromagnetic fields in different occasions [14,15,16,17]. The guideline published in 1998 presents requirements for a frequency range spanning from 0 $\mathrm{Hz}$ to $300 \mathrm{GHz}$ [14]. In 2003, ICNIRP published an additional document containing information regarding procedures for testing compliance with the 1998 guideline, for non-sinusoidal and pulsed fields [15]. The 2010 and 2014 
guidelines revise the recommendations for $1 \mathrm{~Hz}$ to $100 \mathrm{kHz}$ and static fields, respectively $[16,17]$.

Fig. 5 shows that the limits defined in 2010 (blue) are more conservative than the values defined in 1998 (red). The occupational limits (thick lines) are less conservative than the general public limits (thin lines).

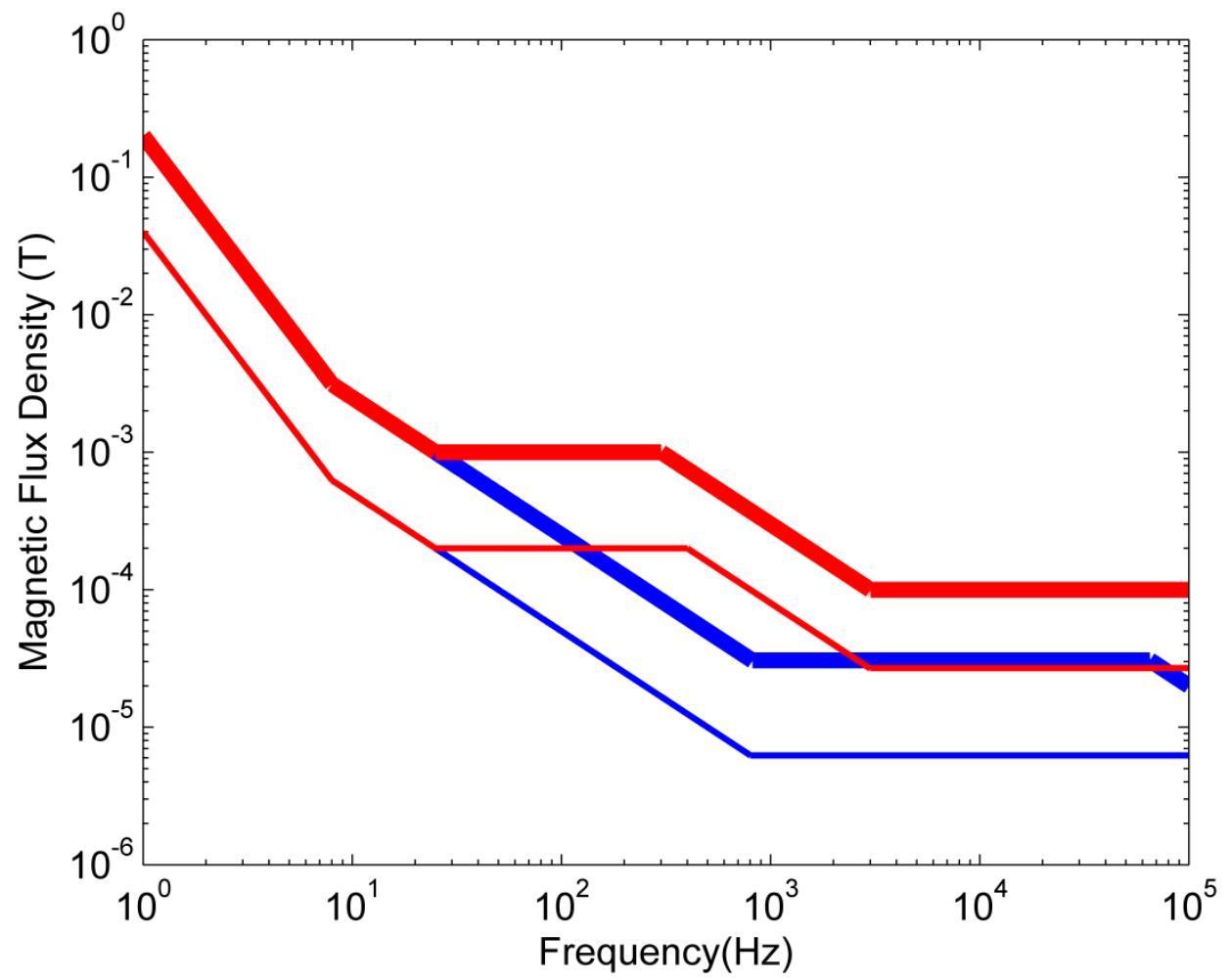

Figure 5 ICNIRP exposure limits comparison. The limits defined in 2010 are in blue, and the limits defined in 1998 are in red. Occupational limits are shown by thick lines and general public limits in thin lines. 


\section{3.}

\section{Therapeutic protocol}

\subsection{1.}

CFM

The Brazilian Federal Council of Medicine is an organ with constitutional attributions of evaluating and standardizing the medical practice in Brazil.

Notably, in 2012, through the resolution 1.986/2012, the board recognized superficial TMS as a private medical act and scientifically proven for use in medical practice nationally, with indications for uni- and bi-polar depressions, auditory hallucinations caused by schizophrenia and neurosurgical planning. Other indications of superficial TMS, as well as deep TMS, were classified as experimental.

The safe parameters for stimulation defined by the CFM were presented in chapter 2 as an example. In chapter 4, further aspects of the therapeutic protocol reliability are analyzed. 


\section{Harmonization of reporting framework and conformity assessment}

\section{1.}

\section{Reporting Framework}

\subsection{1.}

\section{Safe Protocols}

In 1996 a workshop was organized to review the available data regarding the safety of TMS, to allow for the development of guidelines for its safe use [1]. In addition to issues of risk and safety, it also addressed the principles and applications of rTMS, nomenclature, and potential therapeutic effects of rTMS. The guidelines for the use of rTMS cover the ethical issues, recommended limits on stimulation parameters, monitoring of subjects (both physiologically and neuropsychologically), expertise and function of the rTMS team, medical and psychosocial management of induced seizures, and contraindications to rTMS.

Notably, the safe limits for the parameters were compiled into table 1.

Table 1 Data compiled in [1]. Maximum safe duration in seconds of single trains of rTMS based on the National Institute of Neurological Disorders and Stroke experience. Values marked in red are the longest durations tested. No after discharge or spread of excitation has been encountered with single trains of rTMS at these combinations of stimulus frequency and intensity.

\begin{tabular}{|c|c|c|c|c|c|c|c|c|c|c|c|c|c|}
\hline $\begin{array}{c}\text { Frequency } \\
(H z)\end{array}$ & \multicolumn{10}{|c|}{ Intensity } \\
& \multicolumn{10}{|c|}{$\%$ of Motor Evoked Potential (MEP) Threshold) } \\
\hline & $\mathbf{1 0 0}$ & $\mathbf{1 1 0}$ & $\mathbf{1 2 0}$ & $\mathbf{1 3 0}$ & $\mathbf{1 4 0}$ & $\mathbf{1 5 0}$ & $\mathbf{1 6 0}$ & $\mathbf{1 7 0}$ & $\mathbf{1 8 0}$ & $\mathbf{1 9 0}$ & $\mathbf{2 0 0}$ & $\mathbf{2 1 0}$ & $\mathbf{2 2 0}$ \\
\hline $\mathbf{1}$ & 1800 & 1800 & 360 & 50 & 50 & 50 & 50 & 27 & 11 & 11 & 8 & 7 & 6 \\
\hline $\mathbf{5}$ & 10 & 10 & 10 & 10 & 7.6 & 5.2 & 3.6 & 2.6 & 2.4 & 1.6 & 1.4 & 1.6 & 1.2 \\
\hline $\mathbf{1 0}$ & 5 & 5 & 4.2 & 2.9 & 1.3 & 0.8 & 0.9 & 0.8 & 0.5 & 0.6 & 0.4 & 0.3 & 0.3 \\
\hline $\mathbf{2 0}$ & 2.05 & 1.6 & 1.0 & 0.55 & 0.35 & 0.25 & 0.25 & 0.15 & 0.2 & 0.25 & 0.2 & 0.1 & 0.1 \\
\hline $\mathbf{2 5}$ & 1.28 & 0.84 & 0.4 & 0.24 & 0.2 & 0.24 & 0.2 & 0.12 & 0.08 & 0.12 & 0.12 & 0.08 & 0.08 \\
\hline
\end{tabular}


It is worth noting the terminology here used, namely the terms Frequency and Intensity, which compare to Pulse Repetition Frequency and Amplitude as listed by Peterchev et al [37].

A second workshop happened in 2008 with the intentions of updating the previous safety guidelines with more recent literature and available data. A new set of safe limits for the treatment parameters was developed, especially noting the use of repeated TMS protocols, as shown in Table 2.

Table 2 Updated safe parameters compiled in [2]. Safety recommendations for inter-train intervals for 10 trains at $<20 \mathrm{~Hz}$. The maximum duration of pulses for individual rTMS trains at each stimulus intensity should not exceed those listed in the Part B of the table. A consensus has been reached in adopting this table at this point. Values marked in red are the longest durations tested.

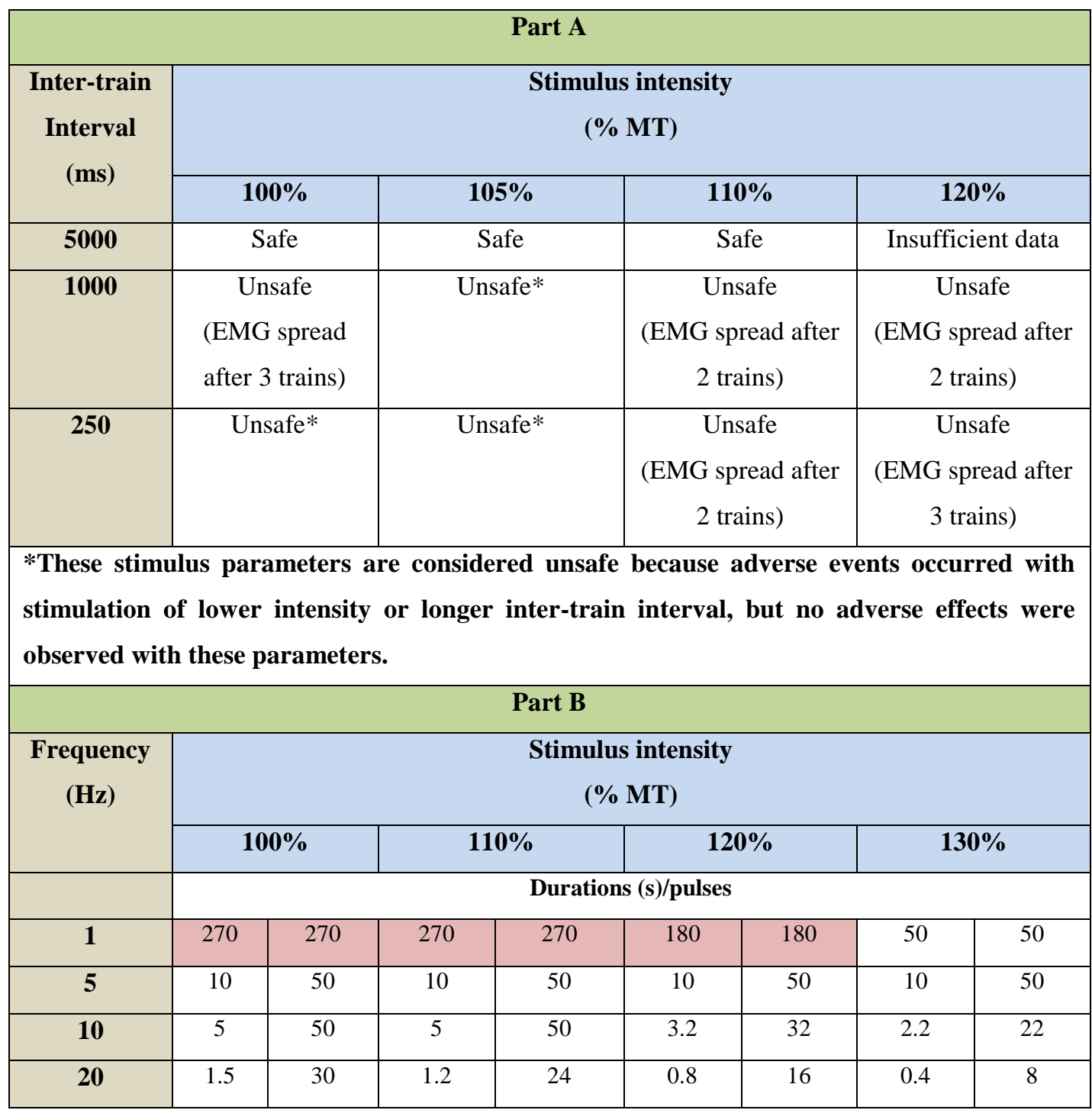




\begin{tabular}{|l|l|l|l|l|l|l|l|l|}
$\mathbf{2 5}$ & 1.0 & 25 & 0.7 & 17 & 0.3 & 7 & 0.2 & 5 \\
\hline
\end{tabular}

The updated guidelines review issues of risk and safety of conventional TMS protocols, address the undesired effects and risks of emerging TMS interventions, the applications of TMS in patients with implanted electrodes in the central nervous system, and safety aspects of TMS in neuroimaging environments. Furthermore, they cover recommended limits of stimulation parameters and other important precautions, monitoring of subjects, expertise of the rTMS team, and ethical issues.

\subsection{2. Treatment Terminology}

Given the great number of quantities necessary to fully describe a given TMS treatment, the terminology used in different publications and documents can become an issue in harmonization. Furthermore, the various protocols of Transcranial Magnetic Stimulation sometimes also receive varying names across different sources.

Namely, after a systematic review of literature and as a result of the 1996 workshop, Wasserman [1] points out that, in an universal system referring to the different types of TMS, the term 'repetitive TMS' should replace the terms 'rapid TMS' and 'rapid-rate TMS' and should be used to refer to regularly repeated TMS delivered to a single scalp site. The term 'fast' or 'high-frequency' rTMS should be used to refer to stimulus rates higher than $1 \mathrm{~Hz}$, and the term 'slow' or 'lowfrequency' rTMS should be used to refer to stimulus rates of $1 \mathrm{~Hz}$ or less. 'Singlepulse TMS' is used to refer to arrhythmical stimulation with conventional magnetic stimulators capable of delivering pulses not more than once every few seconds.

Meanwhile, the 2008 workshop publication [2] went further into this analysis stating that TMS can be applied one stimulus at a time (single-pulse TMS), in pairs of stimuli separated by a variable interval (paired-pulse TMS), or in trains (repetitive TMS). In accordance with the previous workshop [2], this publication also mentioned that, for conventional protocols, there is an universal agreement that the term 'repetitive TMS' (rTMS) has replaced earlier uses of the terms 'rapid TMS' and 'rapid-rate TMS' and should be used to refer to the application of regularly repeated single TMS pulses. The term 'fast' or 'highfrequency' rTMS should be used to refer to stimulus rates of more than $1 \mathrm{~Hz}$, and 
the term 'slow' or 'low-frequency' rTMS should be used to refer to stimulus rates of $1 \mathrm{~Hz}$ or less.

Neither workshop has, however, analyzed further the similar nomenclature inconsistencies or differences in the choices of quantities used to describe the TMS dose delivered.

\subsection{3.}

\section{Treatment Parameters}

Besides the lack of harmonization in terminology on the technique names, documents and publications often describe how the TMS treatment was applied, or which parameters were used, in varying ways, with different degrees of depth.

This non uniformity creates a difficulty in fully describing the dose received by experimental subjects or patients.

Peterchev et al. [37] have published a review in which they list the quantities necessary to fully describe a TMS signal. It divides those into three categories.

Firstly, the quantities related to stimulus waveform parameters. Secondly, those relative to coil configuration parameters, and thirdly, those related to factors for selection of TMS dose.

With respects to the stimulus waveform, the parameters include pulse shape, amplitude, width and polarity; pulse repetition frequency, duration of and interval between bursts or trains of pulses, total numbers of pulses; interval between sessions and total number of sessions. These have been exemplified in fig. 1.

Regarding coil configuration, the parameters include: winding shape and diameter, number of turns in each winding, core dimensions and material, parameters of any auxiliary windings or cores, coil position and orientation on the scalp relative to a reproducible reference frame.

Lastly, regarding TMS dose selection factors, the parameters considered include all available, relevant subject data (e.g., imaging data, pathology reports, relevant physiological measures); experimental or clinical individual response measures (e.g., TMS motor threshold, ECT seizure threshold); summary metrics (e.g., average electrode current density, total charge, total energy); computational models (e.g., electric field or current density field model); prior clinical 
experience; safety considerations (e.g., study exclusion criteria) and methods to normalize functional/clinical responses across individuals.

\subsection{4.}

Framework Harmonization Results

Section 2.3 shows the CFM's recommended values for depressions and hearing hallucinations. Besides the quantities shown on that section, two additional parameters are mentioned in the text. The first is the recommended number of treatment days, which is 20 (or according to evaluation) for depressions and 10 (or according to evaluation) for auditory hallucinations. The second is the application site, which is the right dorsolateral prefrontal cortex for depressions, and the left temporoparietal cortex for auditory hallucinations.

These values are then compared to the ones from the consensus reports [2]. Each publication gives different names for the relevant quantities in the problem, and frequently present different parameters altogether. This makes some degree of interpretation necessary. Table 5 obtained from [2] mentions that an inter-train interval of $5000 \mathrm{~ms}$ is considered safe for stimulation frequencies of less than 20 $\mathrm{Hz}$ with groups of 10 trains. Although CFM recommends a higher number of trains it also allows for a much higher inter-train interval. It then proceeds to say that a quantity referred to as maximum duration of pulses (MDP) for individual rTMS trains should not exceed the values provided on the table. Since one individual train corresponds to a group of pulses, the MDP would correspond to the total duration of said group, which in turn is the very duration of that individual train. Using this interpretation makes the MDP readily comparable to the duration of trains shown in the CFM recommendations. Indeed, these values agree, as can be seen in Table 3 .

Since there were no indications for stimulations at $80 \%$ of motor threshold (MT), the closest comparable value of $90 \%$ was used for this table, as it basically coincides with the values for $100 \%$.

Table 3 Comparison between CFM and Consensus [2] recommendations. CFM values for the pulses in one train are found by dividing the total number of pulses by the total number of trains, and number of treatment days, for the case of depressions. Values related to the maximum duration of pulses taken from tables 4 and 5 from Consensus [2]. 
Numbers in red are the longest value tested up until the point of the 2008 workshop.

\begin{tabular}{|c|c|c|c|c|c|}
\hline $\begin{array}{c}\text { Frequency } \\
(\mathbf{H z})\end{array}$ & $\begin{array}{c}\text { Intensity } \\
(\% \text { of MT) }\end{array}$ & $\begin{array}{c}\text { CFM } \\
\text { duration of } \\
\text { trains } \\
(\mathbf{s})\end{array}$ & $\begin{array}{c}\text { Consensus } \\
\text { MDP } \\
(\mathbf{s})\end{array}$ & $\begin{array}{c}\text { CFM } \\
\text { Pulses per } \\
\text { train }\end{array}$ & $\begin{array}{c}\text { Consensus } \\
\text { Pulses per } \\
\text { MDP }\end{array}$ \\
\hline $\mathbf{1 0}$ & 110 & 5 & 5 & 50 & 50 \\
\hline $\mathbf{5}$ & 120 & 10 & 10 & 50 & 50 \\
\hline $\mathbf{1}$ & $\begin{array}{c}80 \text { or } 100(\mathrm{CFM}) \\
90 \text { or } 100([2])\end{array}$ & 1200 & 1800 & 1200 & $5000^{*}$ \\
\hline \multicolumn{5}{|c|}{ *For intensity of 90\% of MT } \\
\hline
\end{tabular}

As much as there is some ambiguity on how to interpret the name MDP and how it was presented in the Consensus report [2], the duration of trains recommended by CFM and that publication seem to agree. The same happens for the recommended number of pulses per train. However it was necessary to assume that "total number of trains" referred to the entire treatment, considering the 20 days suggested. For treatments of different length in days the number of pulses per train has to be recalculated.

Possibly it could be advantageous to make recommendations directly comparable to the consensus values, using the same quantities such as the MDP, to avoid the need of interpretation, thus preventing ambiguities.

However, harmonization in this case is not something that could be done immediately. For instance, [15], [16] and [31], although in general defining guidelines and directives regarding the same quantities, use different terminology. While ICNIRP publications tend to name relevant exposure quantities as Reference levels for occupational/public exposure to electric or magnetic fields, the EU Directive mentions exposure limit values (ELVs) and action values (AVs). These quantities are comparable, but still have some nuances that require a more careful reading, as the directive also makes a bigger distinction in some tables about different values for limb exposure as opposed to head exposure.

Much more restrictive is the fact that, as mentioned in [15], the ICNIRP reference levels were determined for exposure conditions in which the spatial variation of electric or magnetic fields over the body is small. It is further stated that often the field source is close to the body, making the field non-uniform or very localized, and that standardization bodies have the task to give further 
guidance on the specific exposure situations in which spatial averaging over the body can be applied, as well as deriving new reference levels for special types of non-uniform exposures. It is plausible that rTMS would fall into one of these scenarios, since the fields generated by the stimulation coils are notably not uniformly distributed in space.

This is also noted in [31], in which the European Commission undertakes the task of making available non-binding practical guides that cover, among others, the aspects of spatial averaging of electric and magnetic fields, guidance for dealing with uncertainties in measurements and calculations and most notably guidance on demonstrating compliance in special types of non-uniform exposure in specific situations. Indeed, these documents coincide in several points since the directives in [31] state that they are based on the ICNIRP recommendations.

The user manual for the Neuro-MS system [23] was also analyzed having the previous discussion in mind. It can be obtained using the registration number on the ANVISA website. The reader should be aware that names of some quantities and the symbols for some units do not follow the SI in this document. It uses the obsolete term magnetic induction for the magnetic flux density, and the symbol $\mathrm{Tl}$ for the unit tesla, instead of $\mathrm{T}$, the currently correct one.

With regards to suggesting the terminology and reporting framework necessary for completeness, several publications were found that could serve as a basis for this purpose.

Namely, in [37] a complete list of all relevant quantities intending to fully and satisfactorily define the dosage of a given TMS application is provided. The International Vocabulary of Metrology [38] and the International Electrotechnical Vocabulary [39] can provide a harmonized terminology for much of the terminology used. A notable example would be the usage of the word "amplitude" instead of "intensity" when referring to the amplitude of the TMS pulses, since the term intensity frequently has other meanings not immediately equivalent to the amplitude of a wave [39]. It could be favorable to express the limiting values as a function only of parameters that can be directly adjusted on the devices, to avoid the need of further complicating calculations, when possible.

These observed results provide an outlook of the current situation of rTMS with a focus on safety parameters for Brazil in comparison with worldwide issues. They are signs of a broad encompassing issue of non uniformity regarding 
definition and measurement of relevant parameters in electromagnetic transcranial stimulation dosage and safety as a whole for Brazil. This is also faced in an international level as described in [37].

Research on the calculation and measurement of doses as well as staff exposure should be carried out to provide more data and allow for specific safety requirements.

The aspect of measurement and calculation uncertainty also does not seem to be stressed on the directives and safety standards, and it should be studied to determine how much of a role it could play in correct application of TMS and other modalities. 


\section{2.}

\section{Conformity assessment}

\subsection{1. \\ Suggestion of a harmonized TMS technical standard}

The FDA has published a guidance document aimed specifically at repetitive TMS devices, which was used in the evaluation of more than one system in the United States. The basis for the standard being herein suggested is the structure of the NBR IEC 60601-2-10 standard, used in Brazil for the conformity certification of a given model and brand of TMS device.

Each section (and its subdivisions) of this particular standard was analyzed to verify if it was possible to write an equivalent version if the document was aimed at a TMS device, instead of neuromuscular stimulators.

To obtain the necessary information to fill these sections, other normative documents and guidelines pertaining to the subject of TMS and non-ionizing radiation protection were explored. Notably, the ICNIRP guidelines $[14,15,16,17]$ and the FDA guidance document [40] were found as the most relevant for this case, given their content, scope and objectives.

Furthermore, concepts of the WHO suggestions on frameworks for nonionizing radiation protection were considered, as well as other documents and papers shown to be relevant to the aspect of the metrological reliability of TMS, as discussed by Palatnik-de-Sousa et al. [8,9]

To this end, the terminology and definitions given by the basic metrological documents $[38,41]$ were added so as to strive for harmonization between all parts. Where appropriate, new terms were suggested, always taking this reasoning in consideration.

\subsection{2.}

\section{Conformity assessment harmonization results}

In order to analyze the harmonization of conformity assessment for TMS devices, documents and certificates regarding six TMS equipments from several locations (United States of America, Brazil, Russia and Europe) were studied.

These devices have been labeled in this section as $\mathrm{TMS}_{\mathrm{A}}$ through $\mathrm{TMS}_{\mathrm{F}}$. They are summarized with the corresponding device models and brands in Table 4. 
Table 4 Devices studied in section, with models, brands and corresponding label $\left(\mathrm{TMS}_{\mathrm{A}}\right.$ through $\left.\mathrm{TMS}_{\mathrm{F}}\right)$.

\begin{tabular}{|c|c|c|}
\hline Device & Model & Brand \\
\hline TMS $_{\mathbf{A}}$ & Cerena TMS Device & eNeura Therapeutics \\
\hline TMS $_{\mathbf{B}}$ & Brainsway Deep TMS System & Brainsway \\
\hline TMS $_{\mathbf{C}}$ & Neurostar TMS Therapy & Neuronetics \\
& System & \\
\hline TMS $_{\mathbf{D}}$ & Neuro-MS & Neurosoft \\
\hline TMS $_{\mathbf{E}}$ & MagPro system & MagVenture \\
\hline TMS $_{\mathbf{F}}$ & Axilum Robotics TMS system & Axilum \\
\hline
\end{tabular}

Among the three TMS equipments, $\mathrm{TMS}_{\mathrm{A}}, \mathrm{TMS}_{\mathrm{B}}$ and $\mathrm{TMS}_{\mathrm{C}}$, approved by the Food and Drugs Administration (FDA), compliance to the IEC 60601-1 was observed in $\mathrm{TMS}_{\mathrm{A}}$ and $\mathrm{TMS}_{\mathrm{B}}$. $\mathrm{TMS}_{\mathrm{C}}$ was complying instead with the national/regional equivalents UL/CSA/EN60601-1. $\mathrm{TMS}_{\mathrm{A}}$ and $\mathrm{TMS}_{\mathrm{B}}$ also comply with the collateral standard IEC 60601-1-2 and with a rTMS guidance document by the FDA, with $\mathrm{TMS}_{\mathrm{B}}$ further complying with the ETSI EN 301 489-1 V 1.8 standard, which deals with electromagnetic compatibility for radiofrequencies.

The FDA rTMS guidance $[31,40]$ is a Special Control Guidance for repetitive Transcranial Magnetic Stimulation (rTMS) Systems issued in 2011. This document was developed to support the classification of rTMS devices for the treatment of major depressive disorder into Class II (special controls). The requirements there provided, associated with general controls, are expected to reasonably assure safety and effectiveness of the rTMS systems for the treatment of major depressive disorder in patients who have failed to achieve satisfactory improvement from at least one prior antidepressant medication at or above the minimal effective dose and duration in the current episode, and who are currently not on any antidepressant therapy. Devices $\mathrm{TMS}_{\mathrm{B}}$ and $\mathrm{TMS}_{\mathrm{C}}$ were evaluated regarding their conformity to this document, while device $\mathrm{TMS}_{\mathrm{A}}$ wasn't, probably because it predates the guidance.

In Russia, two certificates for $\mathrm{TMS}_{\mathrm{D}}$ state compliance to a series of Russian standards and documents.

The first certificate for $\mathrm{TMS}_{\mathrm{D}}$ cited the ГОСТ P 50444-1992, a standard regarding general specifications for Medical instruments, apparatus and equipments; and the other three standards have corresponding comparable IEC 
standards of the 60601 family: ГОСТ Р 50267.0-92, ГОСТ Р 50267.0.2-2005, ГОСТ Р МЭК 60601-1-1-2007.

A second certificate of compliance for $\mathrm{TMS}_{\mathrm{D}}$ mentions the documents МСанПиН 001-96, СанПиН 2.2.4.1191-03, ГН 2.1.8/2.2.4.2262-07 and ГН 2.3.3.972-00. Notably the first two are sanitary regulations with limits for the exposure to magnetic fields. They are part of the WHO EMF project database for regulatory documents concerning electromagnetic field safety, for Russia. They deal respectively with electromagnetic fields in occupational environment and indicate permissible values for physical factors during use of domestic articles.

Furthermore, this second certificate of compliance for $\mathrm{TMS}_{\mathrm{D}}$ makes extra suggestions for the safe use of the equipment, and mentions the safe distance that operators should maintain from the TMS coils. Namely, the distance mentioned is $70 \mathrm{~cm}$.

In Brazil, $\mathrm{TMS}_{\mathrm{D}}$ is registered by the national agency of health surveillance and assessed regarding its conformity to several IEC standards: NBR IEC 606011, NBR IEC 60601-1-2, IEC 60601-1-4 and IEC 60601-2-10. The latter is a particular standard for neuromuscular stimulation devices. While on one hand there are no particular standards for TMS devices yet, and this can be seen as an approximation attempt, on the other hand the scope of this standard states that it does not apply to devices and systems intended to stimulation of the brain or neurological research. This further hints at the need for a particular standard for these devices.

$\mathrm{TMS}_{\mathrm{E}}$ and $\mathrm{TMS}_{\mathrm{F}}$ present stated compliance to the Council Directive 93/42/EEC, often cited as the European Medical Device Directive (MDD). This directive applies to medical devices and accessories and, as stated in its Article 2, member states of the European Union shall take all necessary steps to ensure that devices may be placed on the market and/or put into service only if they comply with the requirements laid down on that directive [31].

Furthermore, as stated on Article 5 of MDD, compliance is presumed for devices in conformity with relevant national standards adopted pursuant to the harmonized standards, the references of which have been published in the Official Journal of the European Communities. 
Notably, the IEC 60601 series and particular EN equivalent standards appear among the list of harmonized standards considered on the Official Journal of the European Communities.

Products must comply to the MDD to obtain the CE marking, which is necessary for them to be put into the European market.

Furthermore, in the brochure for $\mathrm{TMS}_{\mathrm{E}}$ it is stated that it complies with the Canadian Medical Device Regulation, the ISO 13485:2003 standard and the US 21 CFR 820.

Over time, the ideal scenario would be that a unified, harmonized particular standard for TMS devices could arise, to be used along with the more general documents. It is worth noting that the FDA rTMS guidance document has several characteristics that could make a good candidate as a basis for this future standard.

The only document to mention the safe distance an operator should maintain from a TMS device is one of the Russian compliance certificates for $\mathrm{TMS}_{\mathrm{D}}$, adopting $70 \mathrm{~cm}$ as safe distance. This is most likely based on the study described in [18], which, in turn, is based on only the MegPro unit with a MCB-70 coil by Medtronic, and on the 1998 and 2003 ICNIRP guidelines [14,20]. However, both the model and brand of the device certified in this case were different than the one studied in [19], and Russian documents follow the ELF frequencies set by СанПиН 2.2.4.1191-03, with different limits as those set by ICNIRP. This fact prompts the need for more studies to confirm this minimum safe distance according to the specificities of the various devices available.

As efforts to unify exposure and emission limits advance (such as those by the WHO EMF Project or similar initiatives), the relevant limits could also be added to the safety and performance requirements of the TMS devices, either on the same particular standard or in a more general standard concerning exposure and emission as a whole.

An analysis of the "Device Description" section of the FDA rTMS guidance shows that all the parameters relevant to characterizing the stimulus waveform and coil configuration, as mentioned in [37], are considered. This indicates that the proposed method of characterization given by this FDA guidance could be used, a priori, as a basis for future standards.

However, uncertainties of measurements are not mentioned in FDA rTMS guidance. This document seems to address this parameter by suggesting the 
representation of an interval of possible values for some quantities (namely pulse width, frequency, pulse train duration and inter-train interval) based on the respective accuracies. It is worth noting that, according to the International Vocabulary of Metrology [38], note 1 of reference number 2.13, accuracy is not a quantity and is not given a numerical value. The FDA document, thus, is most likely referring to either the device's resolution, the measurement error, or measurement uncertainty associated with those quantities.

It would possibly be very beneficial to extend this reasoning to all other quantities involved in this problem, especially the magnetic field intensities of the output, and to use a representation of the associated uncertainties as per the Guide to the Expression of Uncertainty in Measurement [41], while also respecting the VIM terminology, to assure harmonization and avoid ambiguities. This would greatly help in future studies concerning the effect of uncertainties on matters of exposure to non-ionizing radiations and dosage definition, or comparisons with guidelines such as the ICNIRP ones.

The IEC 60601-2-10 standard used on the Brazilian case for $\mathrm{TMS}_{\mathrm{D}}$ conformity assessment could also be considered for possible contributions concerning its structure and possible recommendation of measuring parameters also useful for evaluation of TMS devices, although further adaptation to this type of device is clearly needed. 


\section{5 \\ Simulation}

The magnetic flux density generated by a TMS device has characteristic temporal and spatial distributions that are determined by many of the parameters described on chapter 2 of this dissertation. Some of these parameters can be adjusted on the machine (such as the number of pulses per train, the repetition frequency, among others). Others, such as the number of turns in the emitting coil's winding, or the shape of the emitting coil, are not adjustable, despite still majorly influencing the resulting spatial and temporal characteristics of the magnetic flux density.

The manuals and brochures of the TMS devices and coils often contain some information on the characteristics of the magnetic flux density.

For example, on the Neurosoft model studied, the equipment manuals $[23,24]$ provide several plots with information on the spatial magnetic flux density distribution, and mention values for the time derivative.

However it is not always clear how this data is derived or obtained. On plots presented by Karlström et al. [18] and on the Neurosoft coil manual [24] it is specified that measurements were taken "at the surface" of the coil or at " $2 \mathrm{~cm}$ from the surface", which is a vague description of how measurements could be taken, and this could be easily misinterpreted. Notably, the coil manual focuses more heavily on describing the specifications of several types of coils, whereas the device manual approaches that topic more briefly, giving more attention to the other parts of the device. The spatial distribution of the magnetic flux density presented on the device manual [23], as a function of the distance on the $\mathrm{z}$ axis, does not specify if the data exposed is derived from measurements or from a simulation. 


\section{1. Development of TMS magnetic flux density simulation models}

The two models studied on the simulations were the MCB-70 coil for the MegPro system, because of the availability of both simulated and measured data in literature regarding this coil $[18,20]$, and the AFEC-02-100-C coil for the Neuro-MS system, because it was the model available for measurements, as described at a later point of the study. Both of these models are angulated figureof- 8 coils.

\subsection{1.}

\section{Simulation model for the MCB-70 coil}

The simulation of the magnetic field generated by the MCB-70 and AFEC02-100-C coils were based on several MATLAB procedures that defined the parameters for the calculation of Biot-Savarts's law, the coil and current shapes, among others. The figure-of-eight coil consists basically in a pair of spiral coils $\left(\mathrm{Coil}_{\mathrm{A}}\right.$ and $\left.\mathrm{Coil}_{\mathrm{B}}\right)$ at an angle, with folds towards the centre that allow for the two spiral coils not to cross each other.

All the simulations follow the same axis definition and naming convention for the two windings that form the emitting coil, named $\mathrm{Coil}_{\mathrm{A}}$ and $\mathrm{Coil}_{\mathrm{B}}$.

Figure 6 shows the final shape of the coil used for the simulations, as well as the axes defined. Coil ${ }_{A}$ is located to the left of the figure, slightly above Coil $l_{B}$, which can be seen to the right.

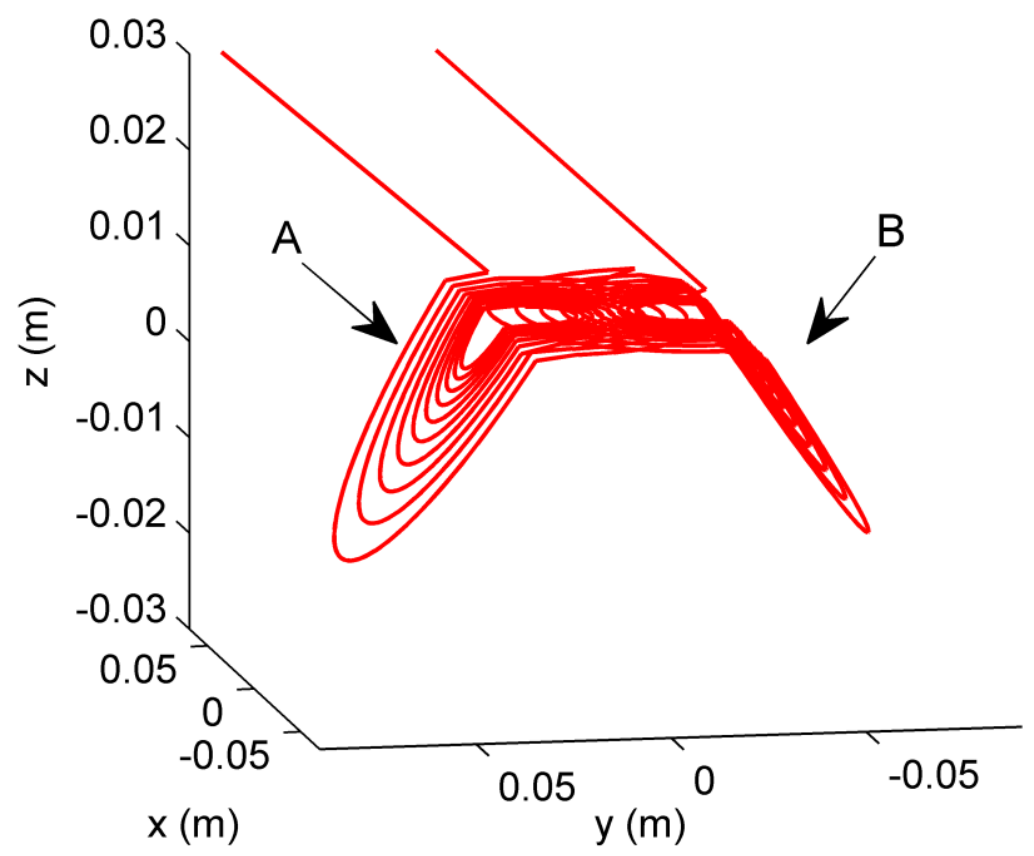


Figure 6 Naming convention for the windings that form the simulated emitting coils. The axes are also defined as shown in the image.

Using the parameters provided in [20], Coil $_{\mathrm{A}}$ and $\mathrm{Coil}_{\mathrm{B}}$ were generated by first using the parametric equations 1 through 3 to define a logarithmic spiral with centre at $(0,0,0)$, inner radius $\left(r_{\text {inner }}\right)$ of $1 \mathrm{~cm}$ and with the variable $p$ being used to ensure an outer radius $\left(r_{\text {outer }}\right)$ of $5 \mathrm{~cm}$ on the $\mathrm{x}-\mathrm{y}$ plane. The number of turns in the coil winding is defined by the variable $n$ such that the total number of turns is 10 .

$$
\begin{aligned}
& x=r_{\text {inner }} e^{\frac{|\theta|}{p}} \cos (-\theta) \\
& y=r_{\text {inner }} e^{\frac{|\theta|}{p}} \cos (-\theta) \\
& z=0 \\
& n=20 \\
& p=\frac{2 \pi n}{\log \left(\frac{r_{\text {outer }}}{r_{\text {inner }}}\right)} \\
& \theta=[-n \pi ; 0]
\end{aligned}
$$

This spiral was then rotated around the $x, y$ and $z$ axes. Namely, the only rotations used in this case were $212^{\circ}\left(32^{\circ}+180^{\circ}\right)$ and $-32^{\circ}$ around the $x$ axis with each value guaranteeing that both coils would end up at a $32^{\circ}$ inclination, so that their generated magnetic fields point inward towards the negative $z$ values following the right hand rule.

The center of the rotated spiral coils then underwent a translation to their final positions, after which a cut-off value set for the $z$ variable defined the height above which they would rotate the same original angle, but backwards, generating the horizontal folds, as seen in Fig. 6.

The full list of parameters defined in the code to generate the coils comprise not only the already cited inner radius, outer radius, coil center coordinates and rotation angles around the three axes, but also the vertical ( $z$ axis) and horizontal ( $y$ axis) distances between the coil centers and the number of turns, which was 10 for both $\mathrm{Coil}_{\mathrm{A}}$ and $\mathrm{Coil}_{\mathrm{B}}$, following [20], and vertical separation between coil centers. 
Namely, the area above the convex side of the coil, towards the positive values of the $z$ axis, will be referred to as "operator side" of the coil, whereas the area below the concave side, towards the negative values of $z$, will be called the "patient side" of the coil.

The code then performs a numerical integration through the path defined by the coil shape, calculating the three components $\left(B_{X}, B_{Y}\right.$ and $\left.B_{Z}\right)$ of the magnetic flux densities with the Biot-Savart's law. The distance between Coil $_{\mathrm{A}}$ and $\mathrm{Coil}_{\mathrm{B}}$ was varied until the spatial distribution of the magnetic flux densities was similar to those presented by [20].

Another procedure was used to define the shape of the current pulse. The electric currents basically consist on trains of single sinusoidal pulses separated by given intervals.

The full list of parameters that define the current shape include: the period (duration) of the sinusoidal pulses, which define their frequency, the amplitude of these pulses, the total duration of the trains, the pulse repetition frequency, the inter-train and inter-pulse intervals and the number of pulses in a train. Notably some of these quantities are fully defined by the values of the others, but were still defined as separate variables to allow for a clearer understanding.

The simulation current consisted on pulses of $7.66 \mathrm{kA}$ amplitude, $286 \mu \mathrm{s}$ pulse duration $(\sim 3.5 \mathrm{kHz}$ equivalent frequency), $5 \mathrm{~Hz}$ pulse repetition frequency, 1 second train duration and 5 seconds inter-train interval. The inter-pulse interval is determined from the variables above.

An adapted version of the Biot-Savart calculating routine then performed the calculations as a function of time, with the same coil shape parameters, but considering the instantaneous value of the current in each iteration of the time loop to calculate the magnetic flux density at a given point in space. The result was the temporal distribution of the magnetic flux density for a point in space specified by the user.

Finally, the time derivative of the magnetic flux density was computed through numerical differentiation in order to obtain the $d B / d t$ signal. Since the magnetic flux density given by the simulation follows the same sinusoidal behavior presented by the current pulses, with the same frequency of about 3.5 $\mathrm{kHz}$, and the same happens for $\mathrm{d} B / \mathrm{d} t$ (with a 90 degree phase caused by the differentiation), one may estimate the behavior of the amplitude of $\mathrm{d} B / \mathrm{d} t$ as a 
function of distance by multiplying the amplitude of $B$ at given distances by the value of the frequency, since the amplitude of the derivative of a sinusoidal function is simply the amplitude of the original function multiplied by its angular frequency.

Both the $\mathrm{d} B / \mathrm{d} t$ pulse shape for this quantity and its amplitude were compared to the experimental results reported in 2006 by Karlstrom et al [18]. Notably, the data from the $\log$ scale $\mathrm{d} B / \mathrm{d} t$ vs distance plot was extracted from the graph published in [18] using a data digitizing software (Gsys) to allow for closer, albeit approximated, comparison with the simulation.

Both sets of data were linearly fitted and the values for the slopes and intercepts were compared. From these it was possible to extrapolate for the occupational exposure safe distance values following the procedure described by Karlstrom et al [18], which mainly consists in considering the $3.5 \mathrm{kHz}$ component of the pulse as the only contribution and using this as a basis to perform the calculations according to the ICNIRP 2003 guidelines [15]. As a comparison, the safe distance was also determined by directly verifying the distance below which the simulation data reached the acceptable value for $\mathrm{d} B / \mathrm{d} t$, eliminating the need for the linear extrapolation.

In a similar manner, the calculations suggested by the 2010 guideline were performed using the simulation data and the result was compared to the safe distance of about $0.7 \mathrm{~m}$ proposed by Karlstrom et al in 2006 [18].

\subsection{2.}

\section{Simulation model for the AFEC-02-100-C Coil}

The simulation for the AFEC-02-100-C figure-of- 8 coil used the same routine as the one for the MCB-70 case, but the variables in the coil equations were changed to reflect the parameters regarding that coil. Several simulations were run making minor alterations until a good fit to the literature data was achieved.

Namely, the inclination angle of the windings is $14^{\circ}$ instead of the $32^{\circ}$ seen on the MCB-70 coil, the vertical and horizontal distances between the winding centers were also changed, and the height at which the windings fold horizontally also changed. 
To account for the plastic chassis surrounding the windings, a small offset in the direction of positive $Z$ (operator's side) had to be assumed. This distance could also be controlled as a variable, and the value that provided the best result was $1.25 \mathrm{~cm}$, which is a reasonable estimate given the dimensions of the emitting coil. This means that even an object directly adjacent to the plastic chassis would still be about $1 \mathrm{~cm}$ away from the windings.

\section{2.}

\section{Simulation results}

\subsection{1.}

\section{Simulation results for the MCB-70}

Figure 7 shows the dependence of the absolute value of $\mathrm{z}$ component of the magnetic flux density $(B)$ with the $y$ coordinate. These values were obtained after varying the coil shape parameters until a satisfactory match to the data in simulation literature [20] was found.

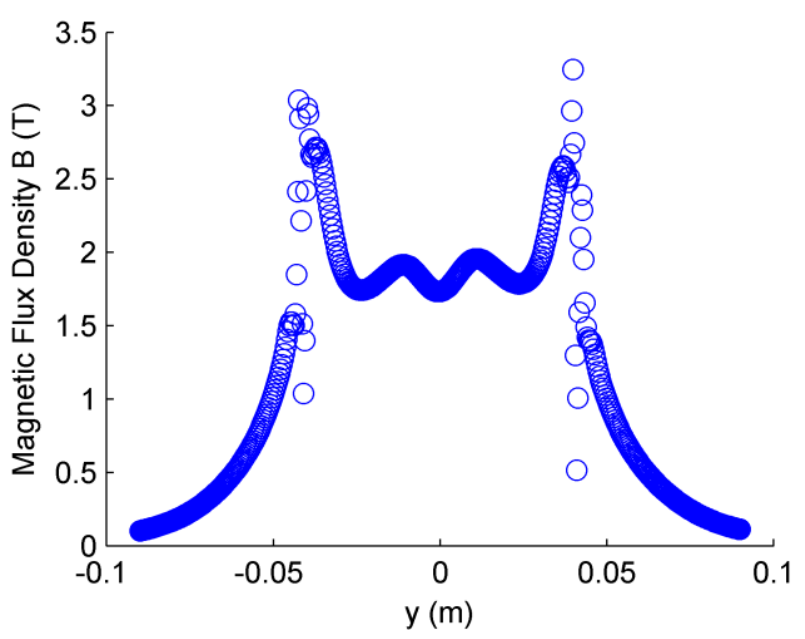

Figure 7 Dependence of the magnitude of the $z$ component of the magnetic flux density with the y coordinate at a distance of $8.3 \mathrm{~mm}$ below the surface of the coil.

By generalizing the calculations to the whole $x-y$ plane, the spatial distribution shown in Fig.7 could be obtained, showing how the magnetic flux densities behave around the coil area. 


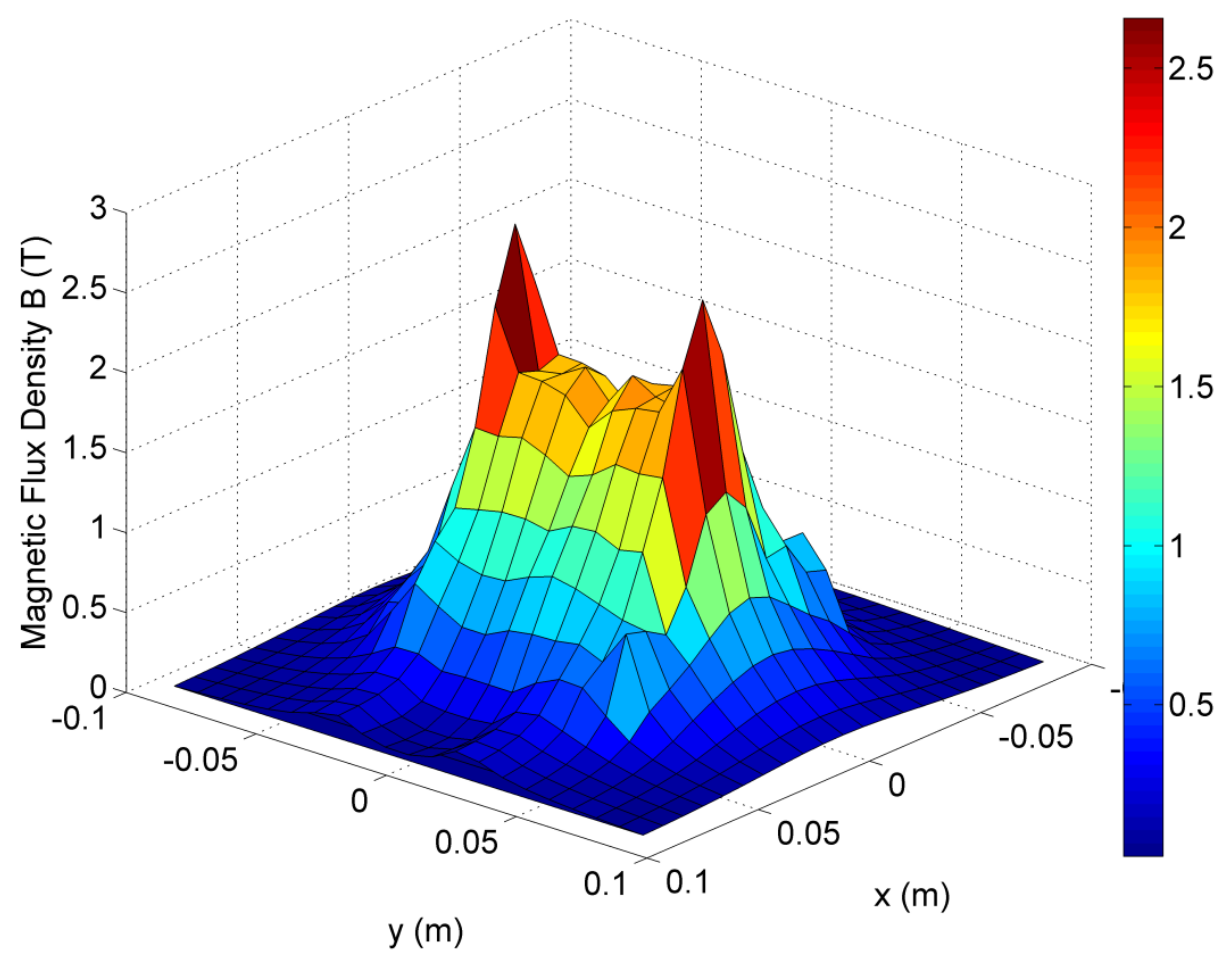

Figure 8 Spatial distribution of $B_{Z}$ on the $x-y$ plane, $8.3 \mathrm{~mm}$ below the surface of the coil.

The comparison between the present simulation data and the experimental results of Karlstrom et al. [18] is shown in Fig.4, where the upper curve (in red) corresponds to the left vertical axis with $\mathrm{d} B / \mathrm{d} t$ values and the lower curve, in blue, corresponds to the right vertical axis with the magnetic flux density values. Both curves are obtained from simulation data. The black squares indicate data extracted from the results of Karlstrom et al. [18].

The safe distances, indicated in Fig.5 by dotted arrows, were calculated using methodologies of the ICNIRP 2003 [15], provided for $\mathrm{d} B / \mathrm{d} t$, and the 2010 guideline [19], for $B$ values, considering that the magnetic field has a $\sim 3.5 \mathrm{kHz}$ (pulse duration of $286 \mu \mathrm{s}$ ) main component that allows disregarding the other components, similarly to the treatment performed by Karlstrom et al. [18].

The safe exposure is, then, associated with a magnetic flux density of $0.1 \mathrm{mT}$, according to the ICNIRP 2010 guideline [16]. This result can be achieved by maintaining a distance of about $1 \mathrm{~m}$ to the operator's side of the coil's surface, as seen by direct inspection of the simulation data shown in Fig.5. 
By computing the base $10 \operatorname{logarithms~of~} \mathrm{d} B / \mathrm{d} t$, of the distances, and of the data provided by [18], a linear fit provided an intercept of 6.05 with a slope of 2.81. The adjustment $\mathrm{R}^{2}$ was 0.9985 .

Using the limit $\mathrm{d} B / \mathrm{d} t$ value of $1 \mathrm{~T} / \mathrm{s}$ considered by Karlstrom et al [18] and recommended by the 2003 ICNIRP guideline [15], one may obtain the safe distance $\left(d_{\text {safe }}\right)$ by extrapolating the linear behavior until reaching the distance where $\log (\mathrm{d} B / \mathrm{d} t)$ has a value of zero (since $\mathrm{d} B / \mathrm{d} t$ has a value of $1 \mathrm{~T} / \mathrm{s}$ ), as can be seen on Fig. 4. From the fitted line equation, one may deduce that this distance is then given by:

$$
\begin{aligned}
& \log \left(\frac{d B}{d t}\right)=\text { Slope } * \log (d)+\text { Intercept } \\
& 0=\text { Slope } * \log \left(d_{\text {safe }}\right)+\text { Intercept } \\
& d_{\text {safe }}=10^{\frac{\text { Intercept }}{\text {-Slope }}}
\end{aligned}
$$

Which gives a safe distance of approximately $1.43 \mathrm{~m}$ for these fitting parameters.

One may also use the simulation data to obtain through direct inspection that distances about $1.32 \mathrm{~m}$ are safe, as seen in Fig.5, without using the linear extrapolation. 


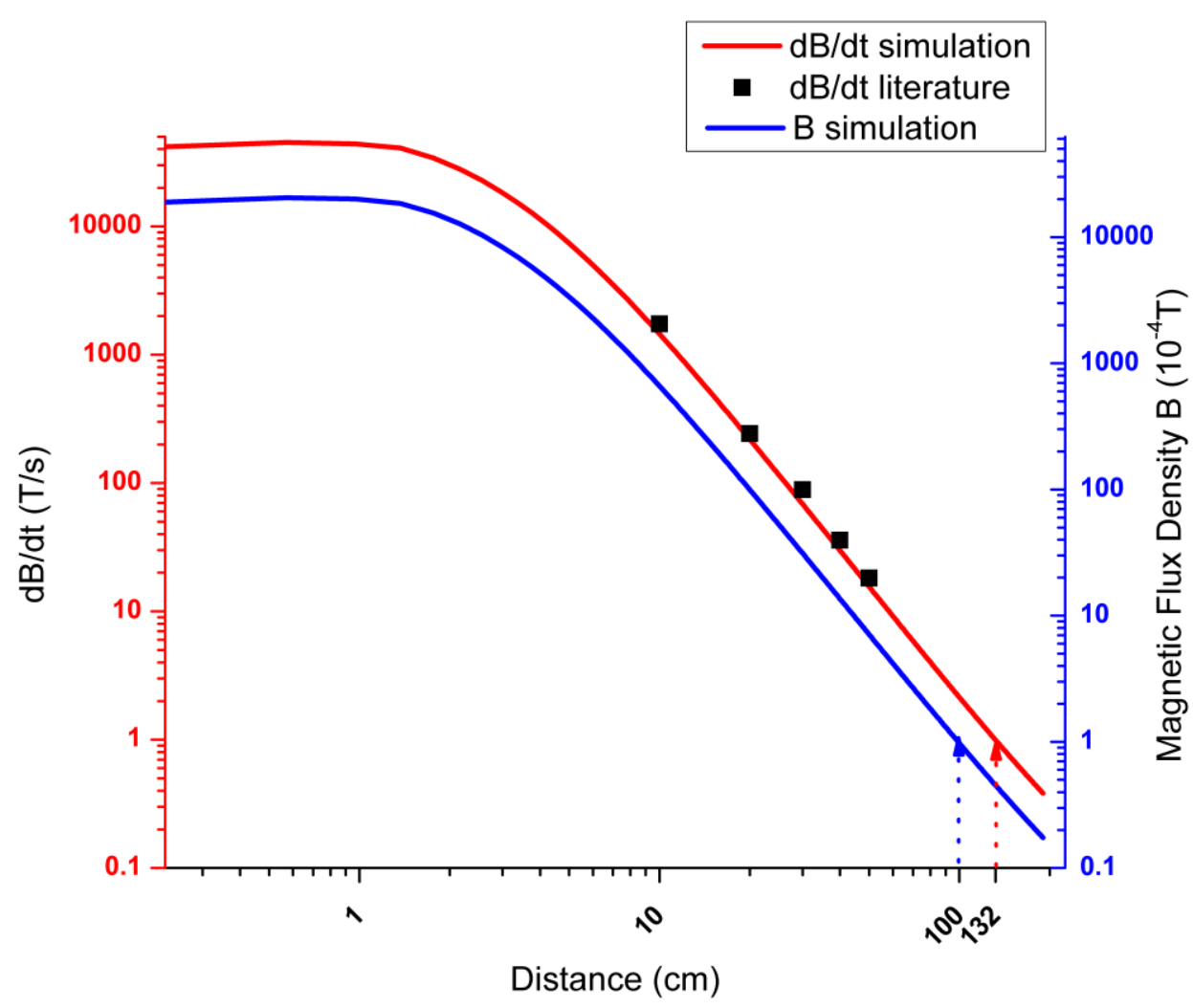

Figure $9 B$ and $d B / d t$ as a function of the distance on the $z$ axis (operator side of the coil). Red and blue curves and axis refer to $\mathrm{dB} / \mathrm{dt}$ and $\mathrm{B}$ values, respectively. Red and blue dotted arrows show the safe distance values obtained by direct comparison of simulation data with the limits of 1 $\mathrm{T} / \mathrm{s}$ and $0.0001 \mathrm{~T}$ provided for $\mathrm{dB} / \mathrm{dt}$ and $\mathrm{B}$ by the 2003 and 2010 ICNIRP guidelines, respectively. The black squares indicate the measured $\mathrm{dB} / \mathrm{dt}$ values available in literature [18] and show how the simulation closely approximates these experimental results.

The dependence of $\mathrm{d} B / \mathrm{d} t$ with the distance to the coil surface (on the operator side) obtained with the present simulation (fig 4), closely resembles the measurement results shown in Fig. 1 of reference [18]. However, the simulated data seems to be offset by about 0.8 to $3 \mathrm{~cm}$ when compared to the measurements.

It is most likely that this stems from the definition of what "at the coil surface" means. This is an expression used both in [18] and [20] and it remains unclear as to how this surface is defined, and also if this definition is used similarly for measurements on both sides (operator and patient).

The simulated fields have to take into account that measurements were most likely performed on the surface of the plastic chassis that surrounds the wire 
winding, and not exactly on the surface of the wires. Offsetting all the simulated data points by about $3 \mathrm{~cm}$ could be justified, then, as a correction to try to consider the influence of the external plastic chassis. Another influence that might factor in would be a systematic error on the definition of the surface level (which would represent a distance of $0 \mathrm{~cm}$ to the coil surface), on the reported measurements. Since the points seem to be offset by a constant value, this could also be a possible explanation for the differences. Thus, it is possible to assume that the simulated data fits the measurements closely, further validating the code. The 2003 and 2010 ICNIRP guidelines $[15,16]$ mention that a spectral analysis is indicated for signals with more than one frequency component. However, the TMS magnetic flux density, over time, has a pulse behavior similar to the current. Since the variation of this quantity happens only during the pulses (being zero elsewhere), and these are cycles of a regular $3.5 \mathrm{kHz}$ sinusoidal wave, this may justify treating the exposure only considering a $3.5 \mathrm{kHz}$ component, as done by Karlstrom et al. [18] and regarding the ICNIRP calculations [15]. Furthermore, the pulses are usually single, whereas the 2010 ICNIRP guidelines define them as having at least five cycles [16], which further justifies leaving the spectral analysis aside, even if this would constitute a first approximation. Future studies might look further into ways of spectrally decomposing this signal to test possible effects of other harmonics, and of the different precautions that might be taken for different pulse repetition frequencies, if necessary. This, however, would require defining how many pulses, or trains of pulses, should be considered for the evaluation.

Using the data extracted from the $\mathrm{d} B / \mathrm{d} t$ vs distance plot in [18], a safe distance of $1.43 \mathrm{~m}$ was obtained through a linear extrapolation. However, by directly inspecting the simulation data shown in Fig.4, $\mathrm{d} B / \mathrm{d} t$ reaches values of about $1 \mathrm{~T} / \mathrm{s}$ at distances around $1.32 \mathrm{~m}$ ( $d_{\text {safe }}$ according to 2003 ICNIRP), and $B$ reaches values of $0.1 \mathrm{mT}$ at about $1 \mathrm{~m}$ ( $d_{\text {safe }}$ according to 2010 ICNIRP). These results show that the most recent guidelines provide a less conservative safe distance for occupational exposure in this case.

Any documents that suggest the safe distance of $0.70 \mathrm{~m}$ (most likely being based on the studies of Karlstrom et al. [18]) should be updated with these new, more conservative, estimates. One example of such a document is the Russian existing certificate of compliance to the СанПиН 2.2.4.1191-03 standard for a 
given TMS device of another brand, different than MC-B70, but also based on a figure-of-eight coil. On that certificate the distance of $0.7 \mathrm{~m}$ is suggested for the device operators.

Despite the difference in models, the presently indicated estimates are the best starting point for occupational safe distance considerations, as they are more conservative. In the future, however, documents regarding the safety of TMS devices should ideally take into account the variations between models, and specific studies for each model would be required for the safe distance suggestions.

\subsection{2.}

\section{Simulation results for the AFEC-02-100-C}

To validate the simulations of the Neurosoft AFEC-02-100-C figure-of- 8 coil, initially, a data digitizing software (Gsys) was used to extract the spatial distribution of the magnetic flux density along the $Z$ axis passing through the center of Coil ${ }_{\mathrm{B}}$ 's winding, as according to [23].

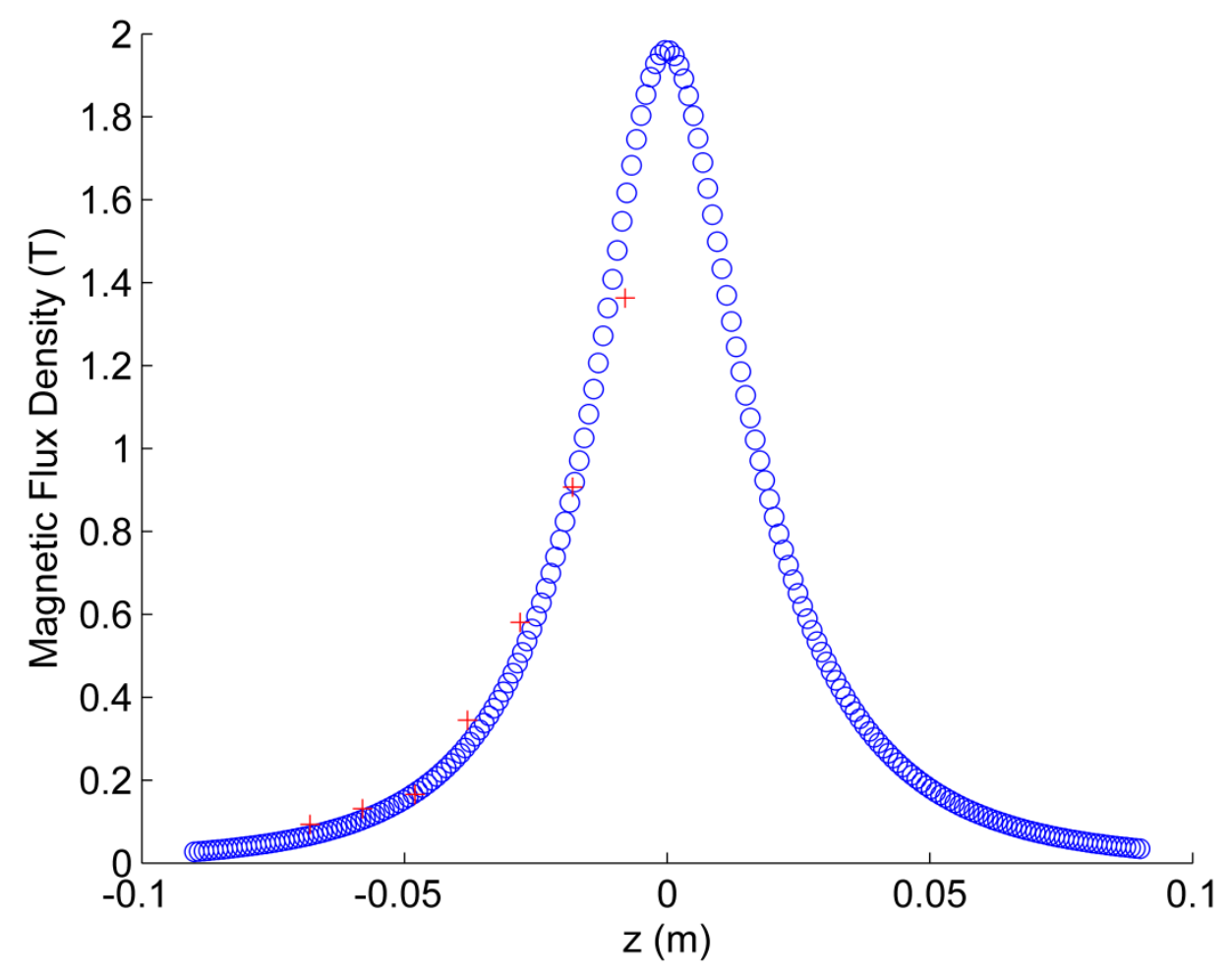

Figure 10 Comparison between the simulation of magnetic flux density on the Neurosoft coil on a vertical line through Coil $_{B}$ center (blue circles) and 
data digitized from [23] (red crosses). The digitized data was offset by $8 \mathrm{~mm}$ on the negative $Z$ direction. Only the $Z$ component of the magnetic flux density is considered.

It became apparent that offsetting the digitized data by $8 \mathrm{~mm}$ caused it to agree strongly with the simulation. This is consistent with the notion that the definition of the $0 \mathrm{~cm}$ height could be ambiguous, especially due to the convex shape of the emitting coil. This offset is equivalent to suppose that whichever sensor was used for that measurement was $8 \mathrm{~mm}$ below the convex shaped surface of the plastic chassis surrounding Coil $_{\mathrm{B}}$.

A flux calculating routine was used to predict the amplitude of the typical voltages the sensor coils would measure. To calculate this, a square grid with sides of $1 \mathrm{~cm}$ was generated, inside of which a circumference with a diameter of $1 \mathrm{~cm}$ was put. The square was then divided in smaller area elements of equal size.

To test how effective this routine would be in calculating the flux precisely, it was noted that the sum of the area elements within the circumference should converge to the area of the circle. The number of elements used was the square of odd numbers to ensure that the center of the circumference would be included in the calculations. Nine hundred and sixty one elements were enough to make the calculated area converge to within the third decimal digit. To ensure higher precision, 10201 elements were used in the effective calculation.

This routine was then paired with the TMS simulation routine and the flux integral was computed for varying distances along the $z$ axis. Namely, there was an interest to know what voltages would be read at very close distances, at the surface of the coil, to ensure that the measurements wouldn't damage the acquisition board. 


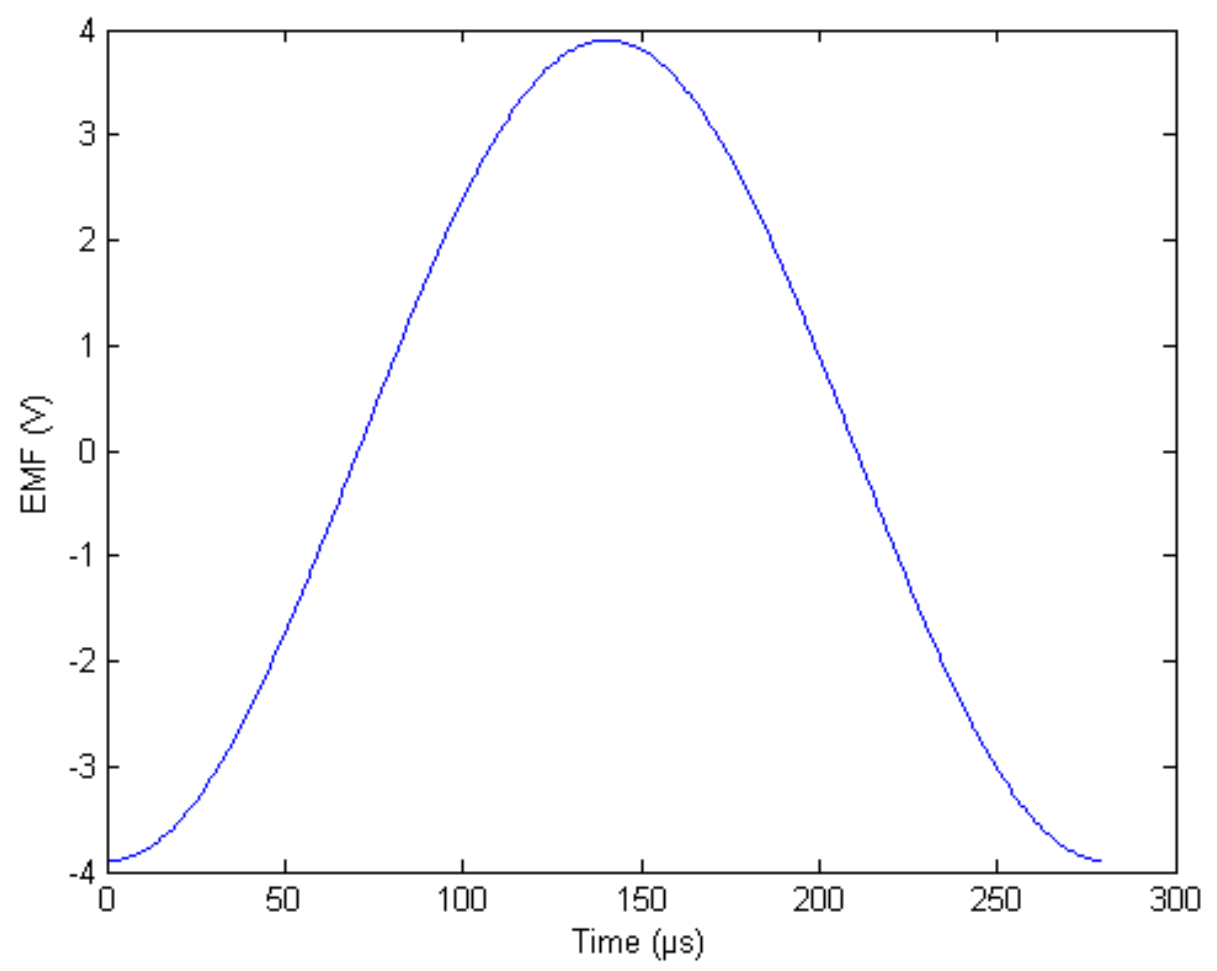

Figure 11 Temporal variation of the voltage levels at the surface of the coil, calculated with 10201 area elements.

Voltage levels of about $4 \mathrm{~V}$ were predicted, which were well within the voltage limits for the NI USB-6229 acquisition board used in this work, without risks of damaging it.

Given that the measurement results seem to confirm the validity of the simulation, a similar approach to the adopted for the MCB-70 coil can be used here to estimate a safe distance for the Neurosoft device, considering the limits defined by ICNIRP. For frequencies of around 2500 to $2800 \mathrm{~Hz}$, the limit magnetic flux density for occupational exposure is $0.1 \mathrm{mT}$, which in the case of the AFEC-02-100-C Neurosoft coil corresponds to a distance of $69 \mathrm{~cm}$ on the patient's side, and $71 \mathrm{~cm}$ on the operator's side, according to the simulation. This is much lower than the simulated estimates for the MCB-70 coil, but although these are two figure-of- 8 angulated models, the AFEC-02-100-C coil has a much less pronounced angle $\left(14^{\circ}\right)$, when compared to the MCB-70 angle of $32^{\circ}$. This could explain why the magnetic flux density falls earlier with distance for this case. This also means that the distances found in this present section should not be 
compared to the ones found by Kalrström et al [18], despite their agreeing values. Instead, they should be compared to the estimates found through the simulations. 


\section{6 \\ Measurements}

\section{1. Development of a measuring system}

The system available for measurements was the Neuro-MS/D Magnetic Stimulator, with the AFEC-02-100-C figure-of- 8 coil. This coil has a typical magnetic flux density output of about 2 teslas, with a derivative of about $40 \mathrm{kT} / \mathrm{s}$ [24].

As these values are too high to be handled by common magnetometers, a new system was developed, based on a NI USB-6229 acquisition board paired with a LabView routine to try and measure the typical fields of a TMS machine. It would only be necessary to develop a transducer in the form of sensor coils in which a voltage was induced due to Faraday's law of induction.

Using this law to estimate a voltage of about $1 \mathrm{~V}$, it was possible to identify an ideal diameter for the sensor coils of about $1 \mathrm{~cm}$. The transducer was decided to be composed by a row of sensor coils, which would allow for simultaneous readings along a given axis, and easier comparison with the data available in literature [18,20,24,23], which are typically measurements along the $Y$ and $Z$ axes.

The routines comprised two different instances: a single channel measurement using the middle sensor coil with an acquisition frequency of 250 $\mathrm{kHz}$ and a multichannel measurement using all 15 sensor coils, with an acquisition frequency of $15 \mathrm{kHz}$ per channel. The single channel measurements, with higher temporal resolution, aimed at obtaining a detailed picture of the pulse shape, whereas the multichannel measurements aimed at mapping the variation of the magnetic flux density with the distance along the $y$ axis.

\section{2. \\ Magnetic Transducer design}

Once the first estimations were made as described in the previous section, there was sufficient data to design a first model for the magnetic sensor coils. 
A LPKF S103 Protomat unit was used to print the sensor coils onto a double-faced copper sheet.

The circuits were designed using the software Eagle CAD 7.3.0. The routing was made to try and minimize the area where any voltage could be induced beyond the main circles comprising the sensor coils.

Once printed, T-blocks were soldered to the routing holes allowing for easier connection of cables. The cable chosen to connect the sensor coils to the acquisition board was a weaved cable, again to help minimize any interference caused by possible induction due to the varying magnetic flux densities during the measurements. This was taken as an additional precaution, despite the fact that the literature and manuals seem to indicate that the fields emitted by the figure-of- 8 coil are highly focalized and decay rapidly outside of a small focus zone $[20,23,24]$.

After many routing design options were analyzed, it was decided that 15 sensor coils with an inner radius of $0.45 \mathrm{~cm}$ and an outer radius of $0.55 \mathrm{~cm}$ would be used. The idea was to maximize the number of coils on the available space in the copper sheet. The spacing between the centers of each sensor coil was $1.2 \mathrm{~cm}$, and the circumferences had a $346^{\circ}$ span. This means that the coils were shy of being perfectly closed circumferences by $14^{\circ}$, which in this case translates to approximately $1 \mathrm{~mm}$, not considering the thickness of the routing paths, which brings this small gap to an even smaller value (small enough to be impossible to measure with a $0.05 \mathrm{~mm}$ resolution caliper).

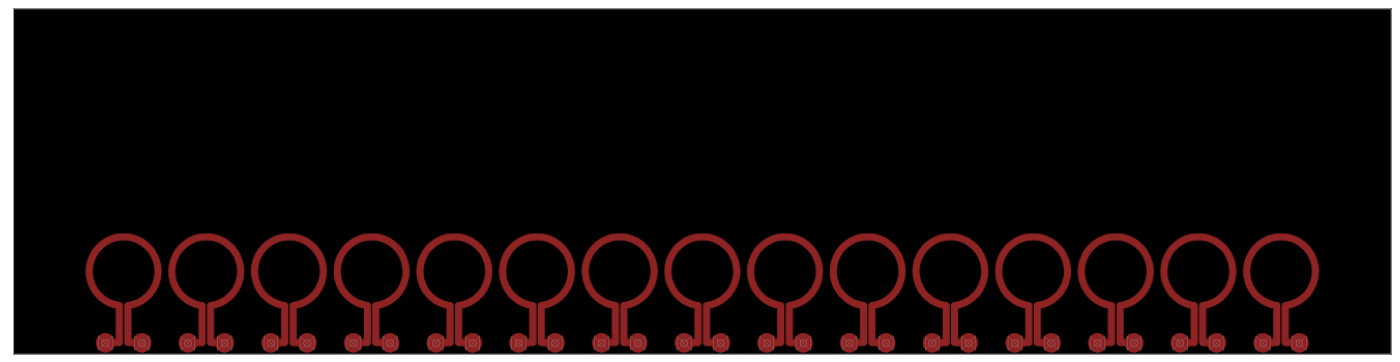

Figure 12 Final magnetic sensor coils design.

These dimensions were considered so that the LPKF S103 Protomat unit could reliably print the circuit onto the copper sheets with the set of drills available. 
Figure 13 Printed sensor coil board.

\section{3 .}

\section{Measurement results}

The measurements performed intended to obtain both the temporal and spatial characteristics of the magnetic flux density emitted. The initial focus was on data that could be readily compared to the data available in literature, in order to validate the simulation results.

The conversion between the voltages read by the acquisition board and the magnetic flux densities that induced those voltages were performed by once again using Faraday's law, under the simplifying assumption that the magnetic flux density did not vary greatly on the span of the area of the sensor coil. As such, and given the geometry of the measurement, the $z$ component of the magnetic flux density, $B_{z 0}$, which was perpendicular to the sensor coil area, could be obtained by the following equations:

$$
\begin{gathered}
\varepsilon=\frac{d}{d t} B_{z} A=\pi r^{2} \frac{d}{d t} B_{z 0} \sin (2 \pi f t) \\
\left|B_{z 0}\right|=\frac{|\varepsilon|}{2 \pi f r^{2}}
\end{gathered}
$$

where $\left|B_{z 0}\right|$ is the absolute value of the amplitude of magnetic flux density's $z$ component, $r$ is the inner radius of the sensor coil, $f$ is the pulse frequency and $\varepsilon$ is the amplitude of the electromotive force (emf) measured.

\subsection{1.}

\section{Single channel measurements}

The single channel measurements were performed using only the central sensing coil of the transducer, at the highest sampling rate available at the acquisition board, which was $250 \mathrm{kHz}$. Using only one channel was necessary in 
order to increase the temporal resolution to the maximum, allowing for a more detailed measurement of the pulse shape.

Using the fact that the simulated field had a local minimum around the center of the coil on the $Y$ direction, there was an attempt to centralize the sensor by searching for this minimum. However, that point is a saddle point and is a local maximum on the $X$ axis, which made this centralization less reliable until a more precise positioning method could be used. As such, a visual centralization was used as a first approximation.

The pulse shape displayed a cosine-like curve, shown in Fig. 14, which was to be expected due to Faraday's law if the magnetic flux density was sinusoidal as a function of time.

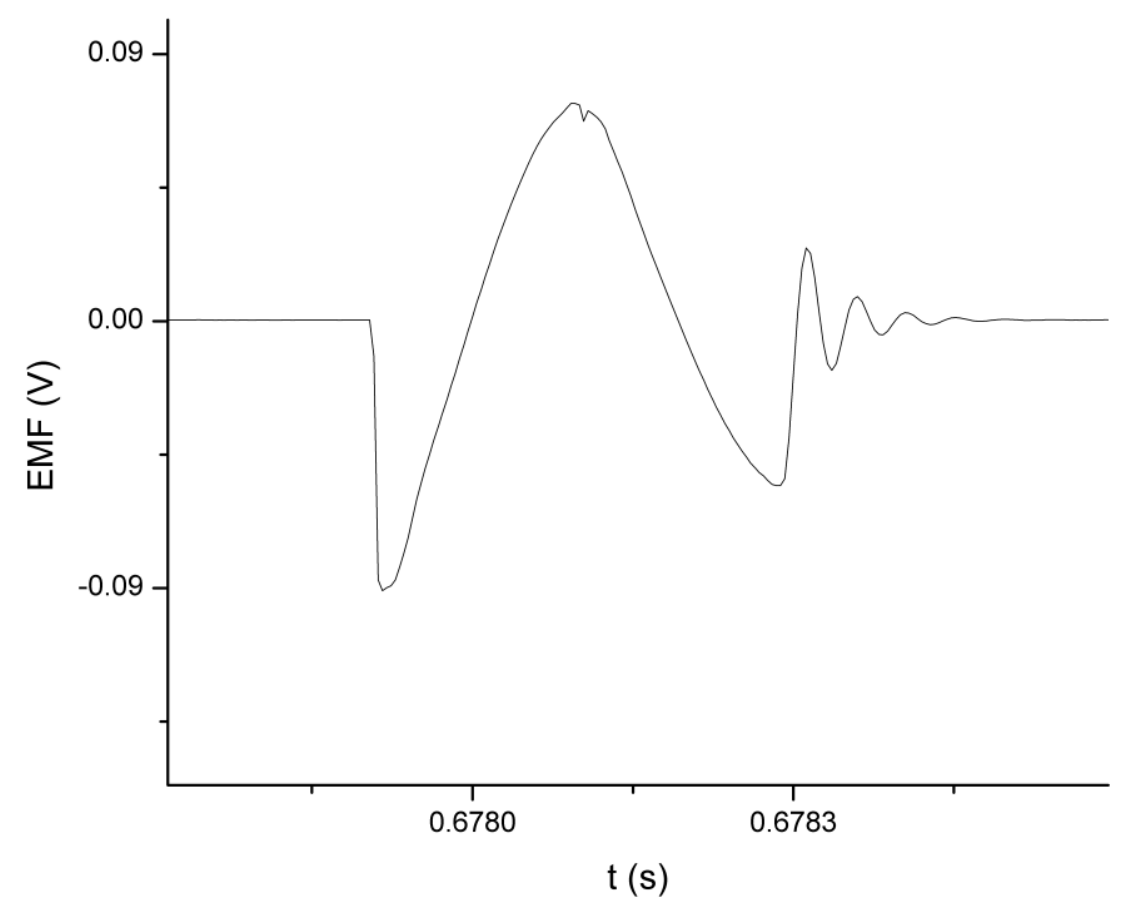

Figure 14 Pulse shape measurement with a single channel, at $250 \mathrm{kS} / \mathrm{s}$, centralized, at $100 \%$ absolute amplitude of the Neuro MS/D system, $1 \mathrm{~Hz}$ pulse repetition frequency, with the emitting coil resting at the top of the sensor coil sheet. Note the cosine-like aspect of the active pulse.

By adjusting a sinusoidal function to the active peak of the pulse, a fit with $\mathrm{R}^{2}$ of 0.97 and a frequency of approximately $2490 \mathrm{~Hz}$ was obtained, as shown in Fig. 14. 


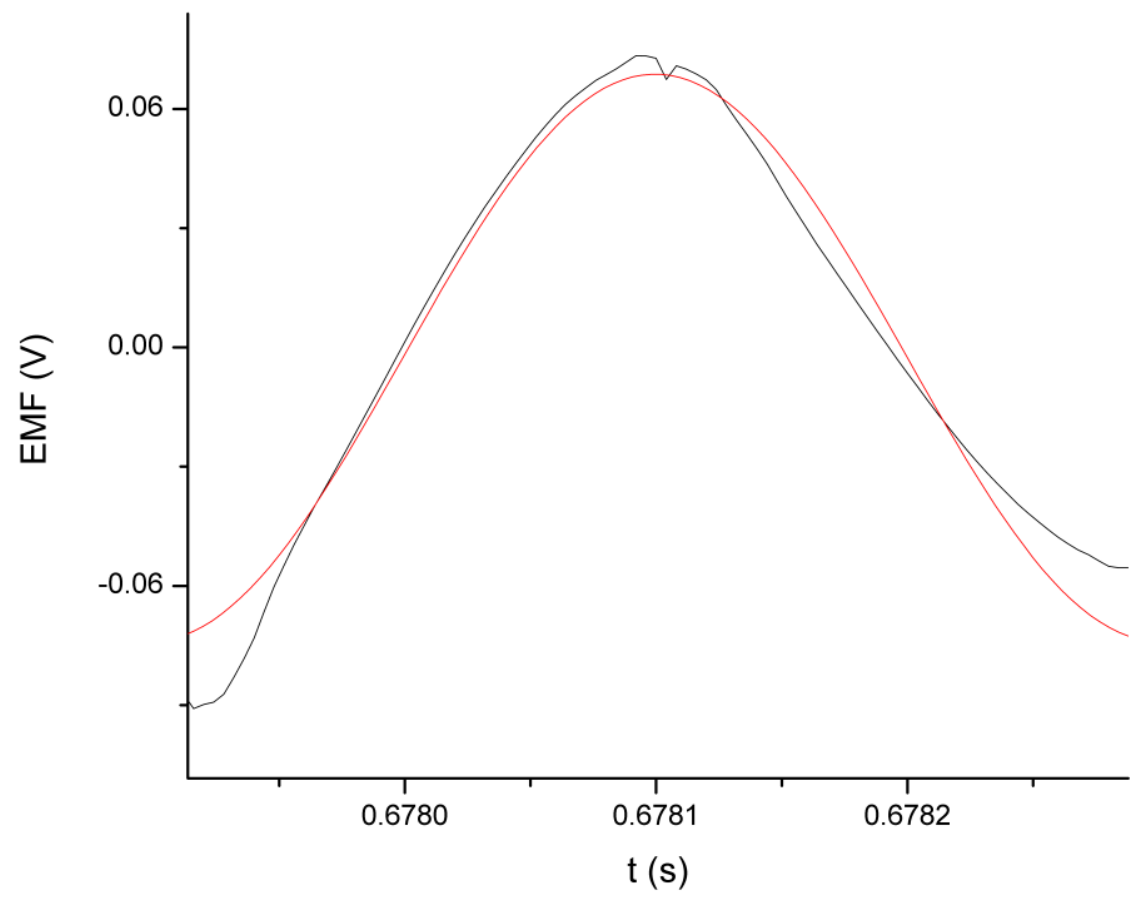

Figure 15 Sinusoidal fit on the active peak of the pulse. The adjusted frequency was found to be approximately $2490 \mathrm{~Hz}$ with an adjusted $R^{2}$ of 0.97 .

Small variations on the data range selection caused the resulting adjusted frequency to oscillate upwards to $2800 \mathrm{~Hz}$. Trying to aid the fit by first integrating the signal so that the sinusoidal shape was more evident increased the $\mathrm{R}^{2}$ to 0.99 , but maintained the frequency around 2560 approximately, as shown in Fig. 15. 


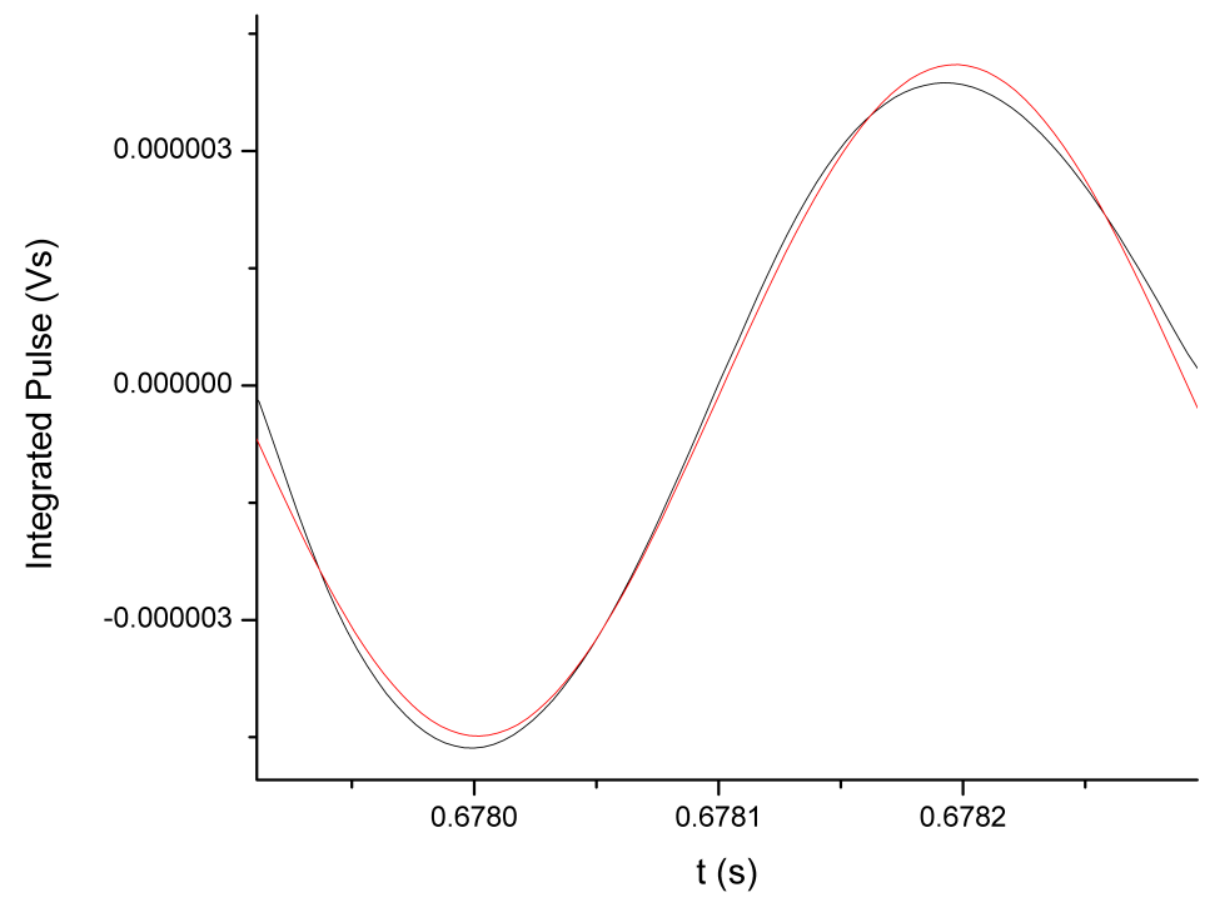

Figure 16 Sinusoidal fit on the active peak of the integrated pulse. The adjusted frequency was found to be approximately $2560 \mathrm{~Hz}$ with an adjusted $\mathbf{R}^{2}$ of 0.99 , indicating a strong sinusoidal behavior.

As a second analysis, a Fast Fourier Transform (FFT) was performed on the same data, yielding a peak in magnitude around approximately $2800 \mathrm{~Hz}$, as shown in Fig. 16. 


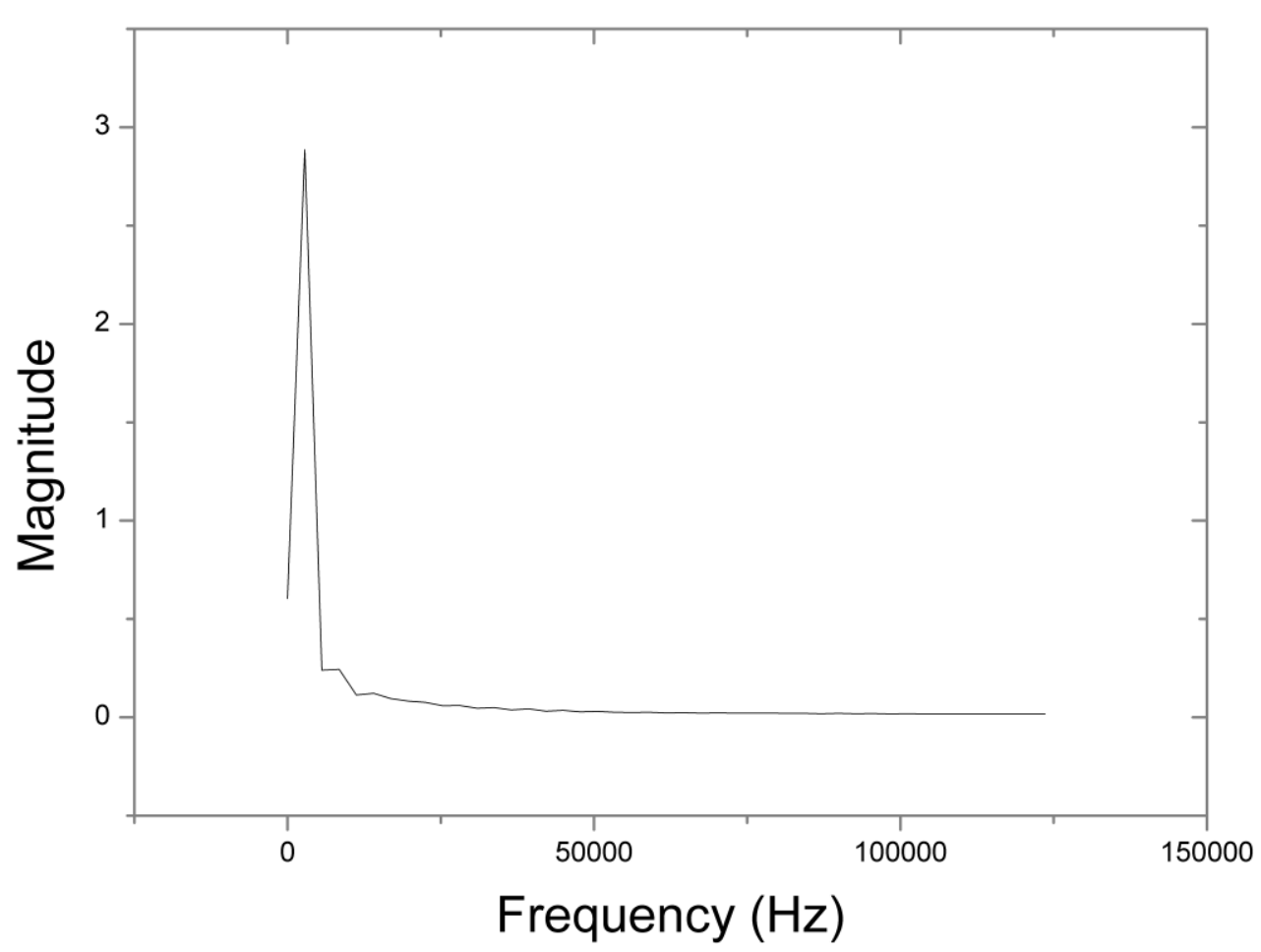

Figure 17 Fast Fourier transform of the active peak of the measured pulse.

\subsection{2. Multi channel measurements}

For the multi channel measurements the centralization procedure adopted was the same. The results were compared to the data extracted from the plots in the coil manual [24], with the Gsys data digitizing software.

Notably the coil's manual does not specify how the measurement was performed, or how "at the coil surface" is defined. Given the convex shape of the emitting coil, it is not trivial to assume exactly what this means, as even a sensor placed immediately in contact with the emitting coil would not be fully in contact with all the points in the surface simultaneously, having different parts of the sensor at different distances from the source of the magnetic flux densities.

The measurements were performed during 10 seconds with a sampling rate of $15 \mathrm{kHz}$ for each of the 15 sensor coils. The TMS device was set at $100 \%$ of the absolute amplitude output, $5 \mathrm{~Hz}$ repetition frequency, 50 pulses per train, which is one of the CFM approved stimulation protocols. 
The multi channel measurements indicated a seemingly periodical variation on the peak values of each pulse, as shown in Fig. 14, however on closer inspection this can be explained by an aliasing effect due to the lower sampling rate required by the multi channel setup. The signal measurement in this case has a sampling frequency of $15 \mathrm{kHz}$, whereas on the single channel measurement they had a $250 \mathrm{kHz}$ sampling rate. With a fundamental frequency of about $2.8 \mathrm{kHz}$ for each pulse, it is likely that several higher harmonics of this fundamental frequency exist in the train of pulses, which could explain the oscillating behavior through Nyquist's theorem. Due to time constraints the measurements were performed without passing the signal throw a low filter pass (with a cut off frequency of around 7.5 and $125 \mathrm{kHz}$ for the multi and single channel measurements respectively ), which may also explain the amplitude variation in the multichannel measurements.

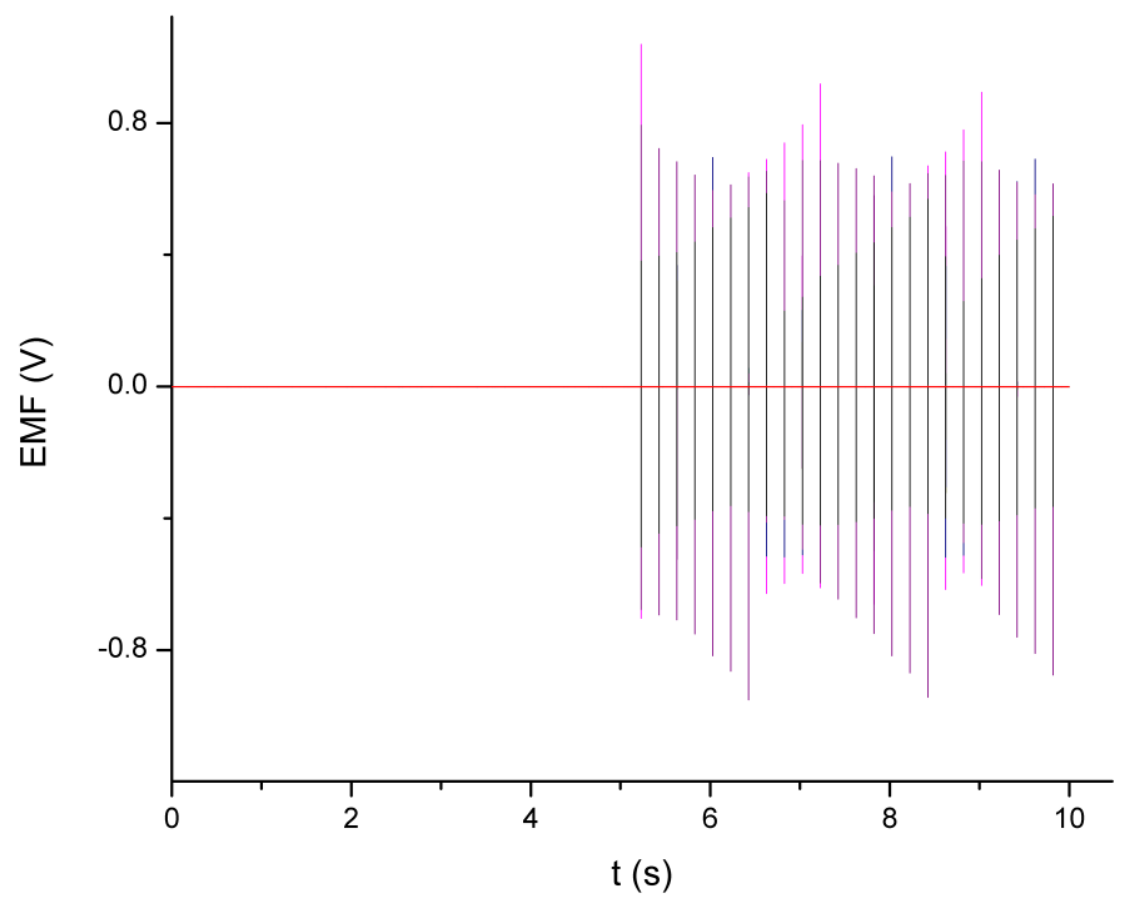

Figure 18 Multichannel measurement with results from the 15 sensor coils superposed. Measurements were taken during 10 seconds at $15 \mathrm{kS} / \mathrm{s}$ for each sensor coil. 
Due to this undersampling, the cosine-like aspect of the curve is not as evident, as shown in Fig. 19. However, since each 10 second measurement had about 24 pulses, some of these had higher peak values, closer to the cosine peak value seen at the single channel measurement. Thus, for the $y$-axis mapping of the magnetic flux densities, the maximum peak value among all pulses measured could be used as a good estimate of the peak magnetic density at that point.

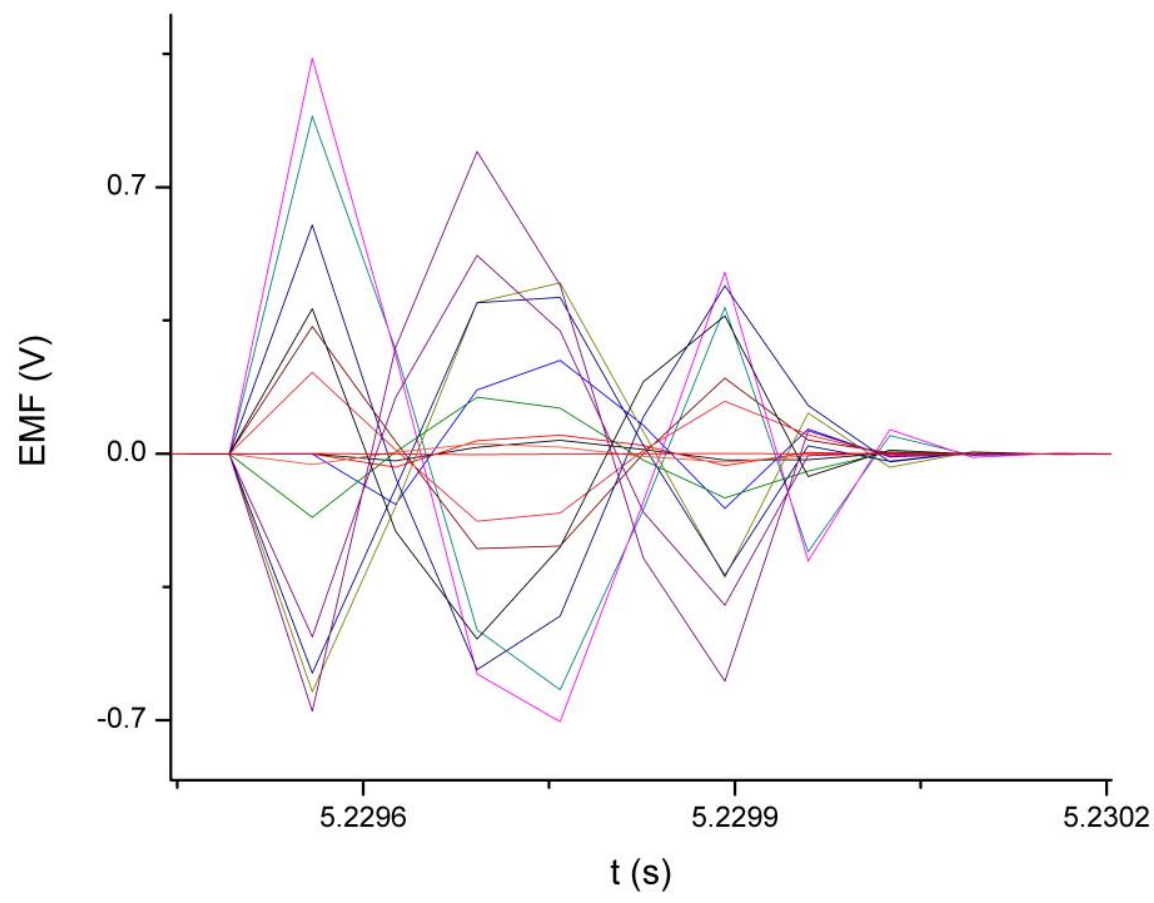

Figure 19 A pulse measured on the multi channel setup. The cosine-like aspect is not as clear due to an aliasing effect caused by undersampling. It is clear that the current goes in opposing directions on each winding, generating magnetic flux densities, and thus induced EMFs in different polarities.

However, the points corresponding to Coil $_{\mathrm{A}}$ showed an inverted polarity, which could be directly interpreted as a sign that the two windings forming the emitting coil were wound in opposite directions, which is an aspect that was not present on the MCB-70 case, where simulating with the windings in the same direction yielded results strongly agreeing with the literature [20]. 
Because of this, for points corresponding to the side of $\mathrm{Coil}_{\mathrm{A}}$, it was necessary to look for the minimum peak values, rather than the maximum, as those points have negative polarities.

Taking the maximum/minimum values for the respective points and plotting them as a function of the distance along the $\mathrm{Y}$ axis, the measured data was compared to data available in the coil manual [24] and to that of the AFEC-02100-C simulation, as shown in Fig. 20. While the measurements did not directly agree with either data set available in the manual, which are measurements made at the surface of the coil (in red), and at $2 \mathrm{~cm}$ of the surface (in blue), they do seem to have the right order of magnitude.

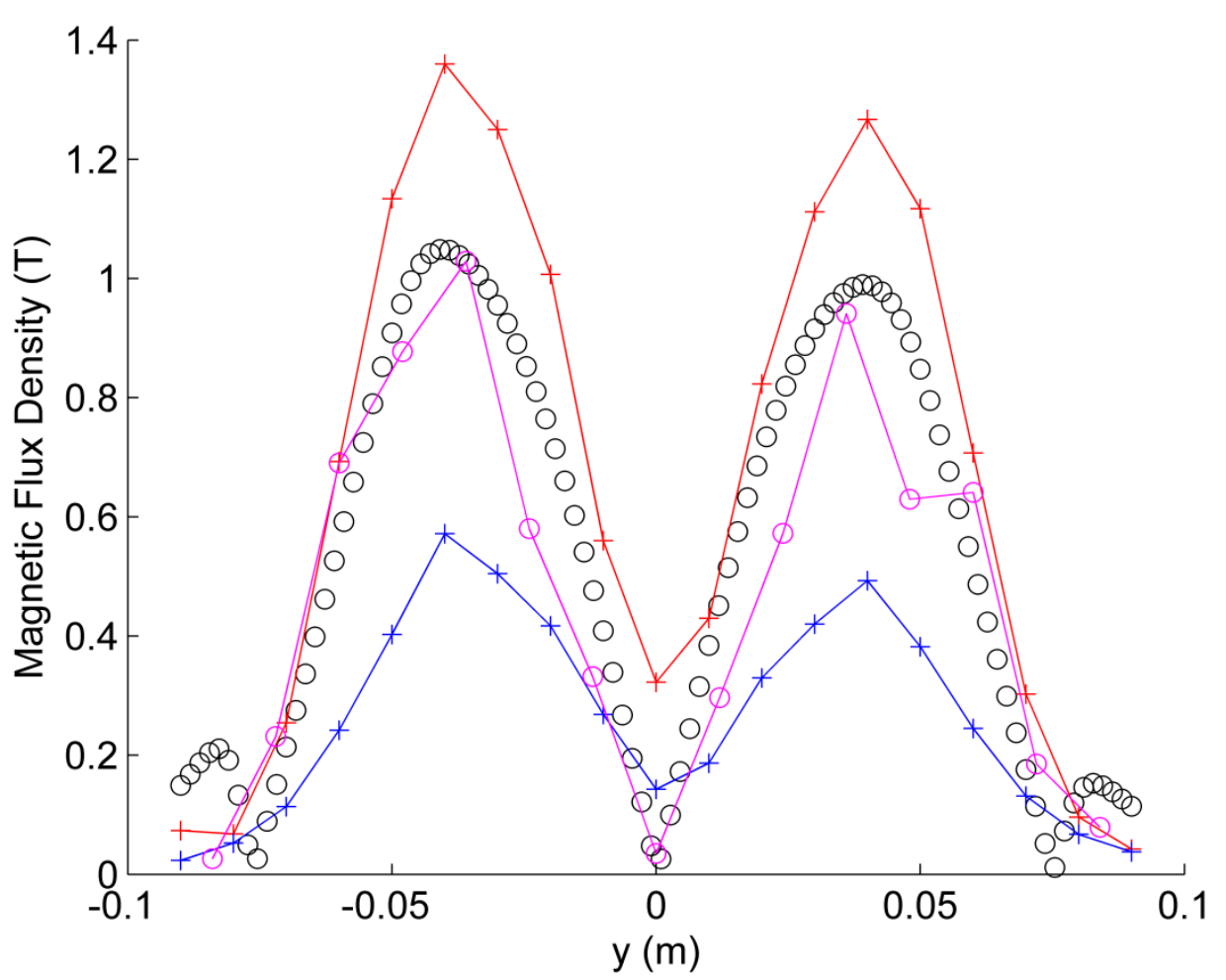

Figure 20 Comparison between the results of measurements made at the surface of the coil (magenta circles) and at $2 \mathrm{~cm}$ from the coil's surface (blue); the data provided by [24] at the coil's surface (red); as well as the simulated data from Matlab routine at $0.5 \mathrm{~cm}$ from the $y$ axis (black circles), along the $z$ axis. Only the $Z$ component of the magnetic flux density is considered.

Furthermore, the simulation predictions for a distance of $0.5 \mathrm{~cm}$ along the $z$ axis agree with the measurement results at the surface of the coil. This suggests an 
ambiguity in how the manual defines what "at the surface of the coil" means. The definition of this origin of the $z$ axis could explain the discrepancies, and the simulation results suggest that. However, the presence of aliasing effects can hinder this interpretation.

These results seem to validate the predictions of the simulation, although there is notable disagreement in the values measured for the center of the coil, at $y=0 \mathrm{~cm}$. As all other points had values between the ones given by [24], one could also expect the same for the point at the center.

The fact that both the simulation and the measurement independently point for a value lower than the one given by the literature in the blue curve raises again the question regarding how "at the surface of the coil" was defined, and as to what kind of measurement was performed to yield the manual's data [24] 


\section{7}

\section{Discussion and conclusion}

\section{1.}

\section{Harmonization of standards and terminology}

The current status of the harmonization of conformity assessment and reporting frameworks relevant to TMS devices and applications was analyzed in this dissertation.

The lack of a particular standard directed specifically at rTMS devices was demonstrated. The results of the present overview concerning the most relevant technical documents used by regulators for conformity assessment of Transcranial Magnetic Stimulation, evaluated for six different TMS equipments used in four regions of the world, indicated a great variability of adopted requirements. This lack of harmonization in conformity assessment emphasizes the need for an international IEC particular standard with appropriate requirements for TMS devices. A suggested basis for this future standard was made using the structures of the FDA guidance document for rTMS devices [40] and the IEC 60601-2-10 standard.

A lack of harmonization in the reporting of results and the characterization of the dose delivered by the equipments was also identified in a range of different publications and document types. This was addressed in this work through the suggestion of a harmonized set of concepts based on consensus literature [37] in the topic, and the relevant metrological and scientific vocabulary $[38,39]$.

The inclusion of the relevant exposure limit requirements could also be considered to the safety and performance requirements of the TMS devices. However, among other aspects, further studies to analyze the completeness of measurement requirements considering the differences of the specificities of the equipments, such as coil configuration, must be performed in order to be addressed in a future particular standard for TMS. 


\section{2. \\ Development of a TMS simulation}

A simulation of the typical magnetic flux densities generated by the MCB70 Medtronic coil was performed in order to validate the simulation routines and to cross validate previous experimental and theoretical results in literature that had not been yet compared to one another. Also, the resulting spatial distribution was used for estimation of safe distances regarding exposure, considering the ICNIRP guidelines $[15,16]$. The results of the simulation were validated through comparison with experimental results available in the literature [18].

Safe distances of $1.43 \mathrm{~m}, 1.32 \mathrm{~m}$ and $1.0 \mathrm{~m}$ for the MCB-70 Medtronic coils were found by three different methodologies. For the first $d_{\text {safe }}(1.43 \mathrm{~m})$ obtained, a linear extrapolation similar to the one performed by Karlstrom et al [18], considering the limit of $1 \mathrm{~T} / \mathrm{s}$ for values of $\mathrm{d} B / \mathrm{d} t$ according to 2003 ICNIRP recommendations [15], was used. For the second $d_{\text {safe }}(1.32 \mathrm{~m})$, a direct inspection of $\mathrm{d} B / \mathrm{d} t$ as a function of distance was evaluated (Fig. 9), also considering the limit of $1 \mathrm{~T} / \mathrm{s}$ given by [15]. The third $d_{\text {safe }}(1.0 \mathrm{~m})$ was obtained by a direct inspection of the values of $B$ as a function of distance (Fig. 9), considering the limit of 0.1 mT given by 2010 ICNIRP recommendations [16].

Since the simulation was validated by comparison with empirical data available in literature [18] and allows predicting the values of $B$ and $\mathrm{d} B / \mathrm{d} t$ for several distances, the estimate given by the linear extrapolation might be overly conservative. Although the $d_{\text {safe }}$ of $1.0 \mathrm{~m}$ found is close to the value of $1.10 \mathrm{~m}$ obtained for both a figure-of-eight and a round TMS coils of non-specified brand in simulations by Lu and Ueno [19], based on 2003 ICNIRP, the value of $1.32 \mathrm{~m}$ is safer and could be the more conservative alternative adopted.

Notably, this value for $d_{\text {safe }}$ is considerably above the value found for the Neurosoft AFEC-100-2-C coil, of about $71 \mathrm{~cm}$. Thus, if a more general value for $d_{\text {safe }}$ should be adopted in official documents dealing with operator safety for TMS devices, until more brands of TMS devices are studied, the usage of the more conservative distance of $1.32 \mathrm{~m}$ for $d_{\text {safe }}$ could enhance safety.

Future studies can validate and enhance this result through experimental measurements of several models coils and brands. This would allow for a 
comparison on how $d_{\text {safe }}$ might vary in those cases and there might be a more appropriate value when considering several different brands.

The fact that the safe distance of $1.32 \mathrm{~m}$ found for the MCB-70 coil is much larger than the one found through the measurements of the AFEC-02-100-C coil can be possibly explained by geometric differences in coil winding shapes, even for similar stimulation parameters. The more pronounced $32^{\circ}$ angles of the MCB70 coil, when compared to the $14^{\circ}$ angles from the AFEC-02-100-C coils, possibly justify the longer distance to fall to safe levels. 


\section{3.}

\section{Development of a measuring system}

A measuring system was successfully implemented and used in preliminary measurements with the Neurosoft Neuro-MS/D TMS device, using the AFEC-02100-C figure-of-8 coil. The system was designed having in mind the typical magnetic flux densities expected and simulated using a Matlab routine. The system basically consisted in a copper sheet with printed sensor coils which acted as transducers, leading the signal to a National Instruments USB-6229 acquisition board.

The single-channel measurements performed provided a clear image of the waveform shape of the pulse, and a sinusoidal fit and a Fast Fourier Transform provided frequency estimates of about $2490 \mathrm{~Hz}$ and $2800 \mathrm{~Hz}$ respectively.

The multi-channel measurements provided a mapping in the $Y$ direction that was comparable to the simulated data, given the proper zero height definition. However to give these results more significance, higher sampling rates should be used in future measurements, together with a set of low pass filters to reduce the effect of aliasing. These initial findings however also hint towards a confirmation of the simulation allowing for a safe distance prediction of about $69 \mathrm{~cm}$ on the patients side and $71 \mathrm{~cm}$ on the operator's side.

These values obtained for the AFEC-02-100-C figure-of- 8 coil (Neurosoft Neuro-MS/D) are consistent with the requirements stipulated by conformity certificates in Russia and with studies by Karlström et al. [18], which concerns the MCB-70 model. Their results were analyzed in this work and proven to be less conservative than they should be, as shown by calculations derived in section 5.2.1.

While the current measurement results do not allow for a detailed quantification of measurement uncertainties, they provide the basis for future projects to develop measuring protocols that minimize measurement errors and uncertainties.

Namely, the current measurements presented in this work already point to the possible systematic errors due to the positioning of the emitting TMS coil and the often ambiguous definition of the origin of the $z$ axis, referred to in at least two publications as "at the surface of the coil" [18] [20]. Similarly, centralizing the 
sensor coils reliably was challenging due to the saddle point nature of the central part of the TMS magnetic flux density. This spatial distribution did not allow for a centralization based on looking for a local maximum reading on the $x$ axis and minimum on the $y$ axis. That could also lead to systematic errors.

Other sources of errors that should be further studied involve the specificities of sensor coil geometries and the calculations used to obtain the values of the magnetic flux density from the electromotive forces measured. The influence of random errors associated with factors internal and external to the TMS device should also be studied in the future, through a large number of repeated measurements for several sets of parameters in identical conditions.

Future works can study these sources of errors and uncertainties in greater detail. 


\section{4. Conclusion}

This work presented a study on the metrological reliability of Transcranial Magnetic Stimulation. The concept of reliability was addressed through three key aspects of safety that were analyzed with a mixed theoretical and experimental approach: safety and performance of the TMS devices; safety regarding exposure limits for patients, staff and the general public; and safety of the therapeutic protocol and of the treatment parameters.

Simulations and measurements were run, as well as a comparison of regulatory documents, in order to cover the analysis of the aspects of safety and performance relevant to this technique and the associated devices.

It was shown that the most basic information needed for an IEC particular standard with specific parameters for TMS evaluation are already available when considering the presently existing documents, most notably the FDA rTMS guideline, the IEC 60601-2-10 standard and the ICNIRP guidelines.

In this way, this work also provides grounds for more detailed performance requirements for future particular standards for TMS devices.

The simulations, validated by the measurements (in the case of the AFEC02-100-C coil) and by literature (in the case of the MCB-70 coil), resulted in estimates for safe distances of about $71 \mathrm{~cm}$ (using the 2010 ICNIRP guideline) for the AFEC-02-100-C coil using the Neuro-MS/D system by Neurosoft, and about $100 \mathrm{~cm}$ (using the 2010 ICNIRP guideline) to $132 \mathrm{~cm}$ (using the 2003 ICNIRP guideline) for the MCB-70 coil simulating the MegPro system by Medtronic Synectics.

The results demonstrate that different models of TMS devices have specific safe distances. This should be taken into account on documents that deal with TMS safety. If looking for a more generic suggestion while further details about the device at hand are not known, the value of $100 \mathrm{~cm}$, using the most recent ICNIRP guidelines, could be used as a first approximation.

Future prospects include further analysis of additional treatment protocols, device brands and coil models, as well as attempting to create a basic draft or groundwork of a possible TMS particular standard. 


\section{8 \\ Bibliographical references}

1 WASSERMAN, E. M. Risk and Safety of repetitive transcranial magnetic stimulation: report and suggested guidelines from the international Workshop on the Safety of Repetitive Transcranial Magnetic Stimulation. Electroencephalography, Jun 5-7, 1996.

2 ROSSI, S.; HALLET, M.; ROSSINI, P. M.; PASCUAL-LEONE, A.; THE SAFETY OF TMS CONSENSUS GROUP. Safety, ethical considerations, and application guidelines for the use of transcranial magnetic stimulation in clinical practice and research. Clinical Neurophysiology, v.120, n.12, p.2008-2039. 2009.

3 PAES F.; MACHADO, S.; ARIAS-CARRIÓN, O.; VELASQUES, B.; TEIXEIRA, S. ; BUDDE, H.; CAGY, M.; PIEDADE, R.; RIBEIRO, P.; HUSTON, J. P.; SACK, A. T.; NARDI, A. E. The value of repetitive transcranial magnetic stimulation (rTMS) for the treatment of anxiety disorders: an integrative review. CNS Neurological Disorders Drug Targets, v.10, 2011.

MACHADO S.; PAES F.; VELASQUES B.; TEIXEIRA S.; PIEDADE R., RIBEIRO P.; NARDI A.; ARIAS-CARRIÓN, O. Is rTMS an effective therapeutic strategy that can be used to treat anxiety disorders? Neuropharmacology, v.62, 2012.

5 COSTA MONTEIRO, E.; LEON, L.F. Metrological Reliability of Medical Devices. Journal of Physics: Conference Series, v.588, 2015.

6 LÓPEZ-IBOR, J. J.; LÓPEZ-IBOR, I. L.; PASTRANA, J. I. Transcranial Magnetic Stimulation. Current Opinion in Psychiatry, v.21, n.5, 2008.

7 CONSELHO FEDERAL DE MEDICINA. Resolução CFM 1.986/2012. 2012. 
8 PALATNIK-DE-SOUSA, I.; MONTEIRO, E. C. Transcranial Magnetic Stimulation in Brazil: A metrological overview of safety and terminology. Proceedings of the XXIV Brazilian Congress in Biomedical Engineering. 2014.

9 PALATNIK-DE-SOUSA, I.; MONTEIRO, E. C. Transcranial Magnetic Stimulation Conformity Assessment. XXI IMEKO, 2015.

10 MONTEIRO, E. C. Biometrologia: confiabilidade nas biomedições e repercussões éticas, Metrologia \& Instrumentação, v.6, 2007.

11 WORLD HEALTH ORGANIZATION (WHO). Environmental Health Criteria 35: Extremely Low Frequency Fields (ELF)". 1984.

12 WHO. Framework for Developing Health-Based EMF Standards. 2006.

13 WHO. http://www.who.int/docstore/peh-emf/EMFStandards/who0102/Worldmap5.htm. Last access: March 14th 2016.

14 INTERNATIONAL COMMISSION ON NON-IONIZING RADIATION (ICNIRP) PROTECTION (ICNIRP). Guidelines for limiting exposure to time-varying electric, magnetic and electromagnetic fields (up to $300 \mathrm{GHz}) .1998$.

15 ICNIRP. Guidance on determining compliance of exposure to pulsed fields and complex non-sinusoidal waveforms below 100 $\mathrm{kHz}$ with ICNIRP guidelines. 2003.

16 ICNIRP. Guidelines for limiting exposure to time varying electric and magnetic fields ( $1 \mathrm{~Hz}-100 \mathrm{kHz}$ ). 2010.

17 ICNIRP. Guidelines for limiting exposure to electric fields induced by movement of the human body in a static magnetic field and by time-varying magnetic fields below $1 \mathrm{~Hz} .2014$.

18 KARLSTRÖM, E. F.; LUNDSTRÖM., R; STENSSON, O.; MILD, K. $\mathrm{H}$. Therapeutic staff exposure to Magnetic Field Pulses During TMS/rTMS Treatments. Bioelectromagnetics, 2006.

19 MAI, L.; SHOOGO, U. Dosimetry of typical transcranial magnetic stimulation devices. Journal of applied physics, 2010.

20 NADEEM, M.; THORLIN, T.; GANDHI, O.; PERSSON, M. Computation of electric and magnetic stimulation in human head 
using the 3-D impedance method. IEEE Transactions on Biomedical Engineering, 2003.

21 PAES, F.; BACZYNSKI, T.; NOVAES, F.; MARINHO, T.; ARIASCARRIÓN, O.; BUDDE, H.; SACK, A. T.; HUSTON, J.P.; ALMADA, L. F.; MAURO, C.; SILVA, A. C.; NARDI, A. E.; MACHADO, S. Repetitive Transcranial Magnetic Stimulation (rTMS) to Treat Social Anxiety Disorder: Case Reports and a Review of the Literature. Clinical Practice \& Epidemiology in Mental Health, v.9, 2013.

22 NEUROSOFT. Neuro-MS/D Transcranial Magnetic Stimulation Brochure.

23 NEUROSOFT. Magnetic Stimulator Neuro-MS Technical Manual.

24 NEUROSOFT. Technical Manual: Coils for the Neuro-MS/D Device.

25 BUREAU INTERNATIONAL DE POIDS ET MESURES (BIPM). Mission, Roles and Objectives. 2013.

INSTITUTO NACIONAL DE METROLOGIA QUALIDADE E TECNOLOGIA (INMETRO). http://www.inmetro.gov.br/inmetro/oque.asp. Last access: 14th march 2016.

27 INMETRO. Certificado de conformidade ELM-9114 - Revisão 01. São Paulo, 2008.

28 INTERNATIONAL ELECTROTECNICAL COMISSION (IEC). International Standard - Medical Electrical Equipment. Part 1: General Requirements for basic safety and essential performance. 3 ed. v.1, 2012.

29 INTERNATIONAL STANDARDIZATION ORGANIZATION (ISO). http://www.iso.org/iso/home/about.htm. Last access: $24^{\text {th }}$ march 2016.

30 ISO. Medical Devices - Quality Management Systems Requirements for regulatory purposes. 2003.

31 EUROPEAN COUNCIL. Council Directive 93/42/EEC concerning medical devices. 1993.

32 ASSOCIAÇÃO NACIONAL DE VIGILÂNCIA SANITÁRIA (ANVISA). http://portal.anvisa.gov.br/wps/portal/anvisa/anvisa/agencia. Last Access: 2016. 
33 ANVISA. Resolução RDC No - 27 de 21 de junho de 2011. 2011.

34 ANVISA. Resolução RDC No - 56 de 6 de abril de 2001. 2001.

35 WHO. Environmental health criteria 238. 2007.

36 WHO. Environmental Health Criteria 232. 2006.

37 PETERCHEV, A. V.; WAGNER, T. A.; MIRANDA, P. C.; NITSCHE, M. A.; PAULUS, W.; LISANBY, S. H.; PASCUAL-LEONE, A.; M. BIKSON, M. Fundamentals of Transcranial Electric and Magnetic Stimulation Dose: Definition, Selection and Reporting Practices. Brain Stimulation, v.5, p. 435-453, 2012.

38 BIPM. International Vocabulary of Metrology. 3. ed. 2012.

39 IEC. Internacional Electrotechnical Vocabulary. 2011.

40 FOOD AND DRUGS ADMINISTRATION (FDA). Class II Special Controls Guidance Document: Repetitive Transcranial Magnetic Stimulation System. 2011.

41 BIPM. Evaluation of Measurement Data, Guide to the Expression of Uncertainty in Measurement. 2008 


\section{9 \\ Annexes}

This section contains the papers generated up to this point from the results of the dissertation. Namely, the papers are "Transcranial Magnetic Stimulation: Terminology and safety aspects concerning therapeutic protocol and device reliability", published on the proceedings of the XXIV Brazilian Congress on Biomedical Engineering, and "TRANSCRANIAL MAGNETIC STIMULATION CONFORMITY ASSESSMENT", published on the proceedings of the XXI International Measurement Confederation (IMEKO. There are three other unpublished papers that were not included in these annexes. 
XXI IMEKO World Congress "Measurement in Research and Industry" August 30 - September 4, 2015, Prague, Czech Republic

\title{
TRANSCRANIAL MAGNETIC STIMULATION CONFORMITY ASSESSMENT
}

\author{
Iam Palatnik de Sousa ${ }^{1}$, Elisabeth Costa Monteiro ${ }^{1}$ \\ ${ }^{1}$ Postgraduate Program in Metrology, Pontifical Catholic University of Rio de Janeiro, Rio de Janeiro, Brazil, \\ iam.palat@gmail.com \\ ${ }^{2}$ Postgraduate Program in Metrology, Pontifical Catholic University of Rio de Janeiro, Rio de Janeiro, Brazil, \\ beth@puc-rio.br
}

\begin{abstract}
A comparison of the current regulatory status of Transcranial Magnetic Stimulation (TMS) devices in several regions of the world is presented. Examples of differences between countries in compliance assessment and certification are analyzed. Harmonization of terminology and reporting, as discussed in the literature, is taken into account, while critical issues are pointed out, aiming at the advancement of the metrological reliability of the technique.

eywords: Transcranial Magnetic Stimulation, Metrological eliability, Safety, Standards, Harmonization

\section{INTRODUCTION}

Transcranial Magnetic Stimulation (TMS) is a technique which a rapidly changing current is passed through a nall coil which is placed on the scalp [1]. The magnetic $\circ$ eld generated by the coil allows getting electric energy ช్ల్ :ross the scalp and skull without the side effects of direct srcutaneous electrical stimulation.

The TMS pulses can depolarize neurons and, when 으 peated pulses are applied, they can modulate cortical citability, depending on the parameters of stimulation. . his has behavioral consequences and therapeutic potential
\end{abstract}

[2]. Typical TMS treatments will use frequencies below 25 $\mathrm{Hz}$ [1], which are often called Extremely Low Frequencies (ELF) [3].

TMS is currently approved by Health Surveillance Agencies of Israel, Canada, New Zealand, Brazil, Australia, United States, European Union, among others [2, 4, 5].

Besides the complete and proper stimulus description, aspects regarding device reliability, including safety and performance checks should be satisfactorily considered [6, 7]. To be registered by health agencies, TMS devices must demonstrate compliance with several technical standards. However, up to the present, no particular standard for TMS devices was published.
The World Health Organization (WHO) has addressed the issue of magnetic field safety in several publications $[8,9]$. Notably, it has encouraged the use of exposure limits defined on the guidelines published by the International Commission on Non-Ionizing Radiation Protection (ICNIRP) [10].

However, institutions in several countries and regions create their own guidelines and standards with differences that range from the terminology used to the actual values of safe limits defined. The WHO has created a database of such standards with the EMF project [11].

The existence of various non harmonized standards implies the possibility of differences in conformity assessment on different regions, sometimes even for the same device.

This work aims at identifying information relevant to the ELF range for TMS devices, allowing for the suggestion of some key aspects for a future particular TMS standard, considering the harmonized standard framework suggested by WHO [8] and the harmonized reporting framework and terminology discussed in recent literature $[12,13]$. Several examples of non harmonized standards and conformity assessment for TMS devices are analyzed in this paper. Furthermore, based on published technical standards and guidelines, this work targets to identify the most relevant documents to be considered in the case of a future development of a technical standard with specific requirements for TMS equipment. 


\section{TECHNICAL GUIDANCE AND REGULATORY DOCUMENTS FOR TMS}

Technical standards, published by the International Electrotechnical Commission (IEC) and the International Organization for Standardization (ISO), provide wideranging requirements for medical devices that are used by regulators for conformity assessment.

The IEC 60601 series, promulgated by the IEC, features several aspects pertaining to the basic safety and essential performance of electrical devices for medical electrical (ME) devices. As such, they aim to specify general requirements and to serve as a basis to particular standards [15].

Notably, collateral standards specify general requirements for basic safety and essential performance applicable to a subgroup of ME equipment or to a specific characteristic of all ME equipment not addressed on the more general IEC 60601-1 document. These can be identified by codes using the format $60601-1-X$.

Furthermore, particular standards (codes with format 60601-2-X) may modify, replace or delete requirements contained in the IEC 60601-1 standard, as appropriate for a given particular type of ME equipment.

Up to this point, there is no IEC particular standard $\varangle$ )ncerning TMS devices [12]. However, there are several smpliance evaluations in which either IEC standards, or tional/regional equivalents UL/CSA/EN60601-1. TMS $_{A}$ id $\mathrm{TMS}_{\mathrm{B}}$ also comply with the collateral standard IEC 60601-1-2 and to a guidance document by the FDA rTMS guidance, with $\mathrm{TMS}_{\mathrm{B}}$ further complying to the ETSI EN 301 489-1 V 1.8 standard, which deals with electromagnetic compatibility for radiofrequencies.

The FDA rTMS guidance [13] is a Special Control Guidance for repetitive Transcranial Magnetic Stimulation (rTMS) Systems issued in 2011. This document was developed to support the classification of rTMS devices for the treatment of major depressive disorder into Class II (special controls). The requirements there provided, associated with general controls, are expected to reasonably assure safety and effectiveness of the rTMS systems for the treatment of major depressive disorder in patients who have failed to achieve satisfactory improvement from at least one prior antidepressant medication at or above the minimal effective those and duration in the current episode, and who are currently not on any antidepressant therapy.

Devices $\mathrm{TMS}_{\mathrm{B}}$ and $\mathrm{TMS}_{\mathrm{C}}$ were evaluated regarding their conformity to this document, while device $\mathrm{TMS}_{\mathrm{A}}$ wasn't, probably because it predates the guidance.
In Russia, two certificates for $\mathrm{TMS}_{\mathrm{D}}$ state compliance to a series of Russian standards and documents.

The first certificate for $\mathrm{TMS}_{\mathrm{D}}$ cited the ГОСТ P 504441992, a standard regarding general specifications for Medical instruments, apparatus and equipments; and the three following having corresponding comparable IEC standards of the 60601 family: ГОСТ P 50267.0-92, ГОСТ P 50267.0.2-2005, ГОСТ Р МЭК 60601-1-1-2007.

A second certificate of compliance for $\mathrm{TMS}_{\mathrm{D}}$ mentions the documents МСанПиН 001-96, СанПиН 2.2.4.1191-03, ГН 2.1.8/2.2.4.2262-07 and ГН 2.3.3.972-00. Notably the first two are sanitary regulations with limits for the exposure to magnetic fields. They are part of the WHO EMF project database for regulatory documents concerning electromagnetic field safety, for Russia. They deal respectively with Electromagnetic fields in occupational environment and with permissible values for physical factors during use of domestic articles.

The limits defined at СанПиН 2.2.4.1191-03 encompass the range of low frequency fields relevant to TMS devices (0-25 Hz, approximately). The requirements, however, are different to the limits for the same frequencies given by issued ICNIRP guidelines.

Furthermore, this second certificate of compliance for $\mathrm{TMS}_{\mathrm{D}}$ makes extra suggestions for the safe use of the equipment, and mentions the safe distance operators should maintain from the TMS coils. Namely, the distance mentioned is $70 \mathrm{~cm}$.

In Brazil, the $\mathrm{TMS}_{\mathrm{D}}$ is registered by the national agency of health surveillance and assessed regarding its conformity to several IEC standards: NBR IEC 60601-1, NBR IEC 60601-1-2, IEC 60601-1-4 and IEC 60601-2-10. The latter is a particular standard for neuromuscular stimulation devices. While on one hand there are no particular standards for TMS devices yet, and this can be seen as an approximation attempt, on the other hand the scope of this standard states that it does not apply to devices and systems intended to stimulation of the brain or neurological research.

This further hints at the need for a particular standard for these devices.

The $\mathrm{TMS}_{\mathrm{E}}$ and $\mathrm{TMS}_{\mathrm{F}}$, present stated compliance to the Council Directive 93/42/EEC, often cited as the European Medical Device Directive (MDD). This directive applies to medical devices and accessories, and as stated on its Article 2, member states of the European Union shall take all necessary steps to ensure that devices may be placed on the market and/or put into service only if they comply with the requirements laid down on that directive [16].

Furthermore, as stated on Article 5 of MDD, compliance is presumed for devices in conformity with relevant national standards adopted pursuant to the harmonized standards, the references of which have been published in the Official Journal of the European Communities.

Notably, the IEC 60601 series and particular EN equivalent standards appear among the list of harmonized standards considered, on the Official Journal of the European Communities.

For products to obtain the CE marking necessary to be put into the European market, they must comply to the MDD.

Furthermore, in the brochure for $\mathrm{TMS}_{\mathrm{E}}$ is stated that it 
complies with the Canadian Medical Device Regulation, the ISO 13485:2003 standard and the US 21 CFR 820.

\section{EXPOSURE LIMITS}

The International Comission on Non Ionizing Radiation Protection (ICNIRP) has issued guidelines for protection against non ionizing radiation [10, 17, 18]. The 1998 guideline has requirements for a frequency range spanning from $0 \mathrm{~Hz}$ to $3 \mathrm{GHz}$, with the 2010 and 2014 guidelines revising the recommendations for $1 \mathrm{~Hz}$ to $1 \mathrm{kHz}$ and static fields, respectively. In 2003 there was also an additional document with information for measurement procedures regarding testing compliance with the 1998 guideline [19].

Considering that TMS is a technique which involves delivering a dose of non ionizing radiation, several aspects are raised regarding exposure and associated definitions and concepts.

The ICNIRP guidelines can be used as a basis to determine which distances would be within occupational safe limits considering the exposure to the fields emitted by the TMS device, for operators [20,21].

In [20], by means of measurements performed with a calibrated coil, following the 2003 ICNIRP guidance, a safe ristance of $70 \mathrm{~cm}$ was indicated. In [21], using a phantom to $\varangle$ udy the exposure, the identified safe distance was $110 \mathrm{~cm}$. is worth noting that these two publications did not $\underset{\mathcal{N}}{*}$ msider the same brand and model of TMS device for their Ð udies.

\section{REPORTING FRAMEWORK}

Another aspect relevant to the discussion of TMS safety is at of a harmonization of terminology and the formulation a consistent reporting framework. The idea of discussing hich parameters fully characterize the use of TMS was ready present in the publication of safety recommendations ated by an international consensus [2].

The publications that can be used as a basis for the irmonization of relevant terminology and definitions for this matter, if and when a particular standard for TMS devices arise, are discussed in [12].

Notably, in [14] a complete list of all relevant quantities intending to fully and satisfactorily define the dosage provided in a TMS application is provided.

\section{DISCUSSION}

Over time, the ideal scenario would be that a unified, harmonized particular standard for TMS devices could arise, to be used along with the more general documents. It is worth noting that the FDA rTMS guidance document has several characteristics that could make a good candidate as a basis for this future standard.

The single document to mention the safe distance an operator should maintain from a TMS device is one of the Russian compliance certificates for $\mathrm{TMS}_{\mathrm{D}}$, adopting $70 \mathrm{~cm}$ as safe distance. This is most likely based on the study described in [20], which, in turn, is based on only one brand and model of TMS device, and on the 1998 and 2003
ICNIRP guidelines $[10,19]$. However, both the model and brand of the device certified in this case were different to the one studied in [20], and Russian documents follow the ELF frequencies set by СанПиН 2.2.4.1191-03, with different limits as those set by ICNIRP. This fact prompts the need for more studies to confirm this minimum safe distance according to the specificities of the various devices available.

As efforts to unify exposure and emission limits advance (such as those by the WHO EMF Project or similar initiatives), the relevant limits could also be added to the safety and performance requirements of the TMS devices, either on the same particular standard or in a more general standard concerning exposure and emission as a whole.

An analysis of the "Device Description" section of the FDA rTMS guidance shows that all the parameters relevant to characterizing the stimulus waveform and coil configuration, as mentioned in [14] are considered. This indicates that the proposed method of characterization given by this FDA guidance could be used, a priori, as a basis for future standards.

However, uncertainty of measurements are not mentioned in FDA rTMS guidance. This document seems to address this parameter by suggesting the representation of an interval of possible values for some quantities (namely Pulse width, Frequency, Pulse train duration and Inter-train interval) based on the respective accuracies. It is worth noting that according to the International Vocabulary of Metrology (VIM) [22], note 1 of reference number 2.13, accuracy is not a quantity and is not given a numerical value. The FDA document, thus, is most likely referring to either the device's resolution, the measurement error, or measurement uncertainty associated with those quantities.

It would possibly be very beneficial to extend this reasoning to all other quantities involved in this problem, especially the magnetic field intensities of the output, and to use a representation of the associated uncertainties as per the Guide to expression of Uncertainty in measurement [23], while also respecting the VIM terminology, to assure harmonization and avoid ambiguities. This would greatly help in future studies concerning the effect of uncertainties on matters of exposure to non ionizing radiations and dosage definition, or comparisons with guidelines such as the ICNIRP ones.

The IEC 60601-2-10 standard used on the Brazilian case for $\mathrm{TMS}_{\mathrm{D}}$ conformity assessment, could also be considered for possible contribuitions concerning its structure and possible recommendation of a measuring parameter also useful for evaluation of TMS devices.

\section{CONCLUSIONS}

The results of the present overview concerning the most relevant technical documents used by regulators for conformity assessment of Transcranial Magnetic Stimulation, evaluated for six different TMS equipments used in four regions of the world, indicated a great variability of adopted requirements. This lack of harmonization in conformity assessment emphasizes the need for an international particular standard for these 
devices, with appropriate requirements for TMS devices.

Technical documents such as FDA rTMS guidance, IEC 60601-2-10, as well as the Russian standards and aspects already discussed in literature should be considered in a future possible international standard with specific parameters. The inclusion of the relevant exposure limits requirements could also be considered to the safety and performance requirements of the TMS devices. However, among other aspects, further studies to analyse the completeness of measuring requirements considering the differences of the specificities of the equipments, such as coil configuration, must be performed in order to be addressed in a future particular standard for TMS.

\section{ACKNOWLEDGMENTS}

We thank the Brazilian funding agency $\mathrm{CNPq}$ for financial support.

\section{REFERENCES}

[1] E. M. Wasserman, Risk and Safety of repetitive transcranial magnetic stimulation: report and suggested guidelines from the international Workshop on the Safety of Repetitive Transcranial Magnetic Stimulation, June 5-7, 1996. Electroencephalography and clinical Neurophysiology. 108, 1-16. 1998

S. Rossi, M. Hallet, P. M. Rossini , A. Pascual-Leone, The Safety of TMS Consensus Group, Safety, ethical considerations, and application guidelines for the use of transcranial magnetic stimulation in clinical practice and research. Clinical Neurophisiology vol.120 (12), 2008-2039. 2009.

WHO - World Health Organization "Environmental Health Criteria 35: Extremely Low Frequency Fields (ELF)". 1984.

] J. J. López-Ibor, I. L. López-Ibor, J. I. Pastrana, "Transcranial Magnetic Stimulation. Current Opinion in Psychiatry". vol. 21, 1-5, 2008.

Conselho Federal de Medicina, Resolução CFM 1.986/2012.

[6] E. Costa Monteiro, "Biometrology: reliability of biomeasurements and ethical implications", "Biometrologia: confiabilidade nas biomedições e repercussões éticas", Metrologia e Instrumentação, vol. 6, pp. 6-12, 2007.

[7] E. Costa Monteiro, L. F. Leon "Metrological Reliability of Medical Devices", Conference Series in press.

[8] WHO - World Health Organization, "Framework for Developing Health-Based EMF Standards". 2006.

[9] WHO - World Health Organization, "Model Legislation for Electromagnetic Fields Protection". 2007.

[10] The international Comission on Non-Ionizing Radiation Protection, Guidelines for limiting exposure to time-varying electric, magnetic and electromagnetic fields (up to $300 \mathrm{GHz}$ ). Health Physics 74 (4): 494$522 ; 1998$
[11] http://www.who.int/docstore/peh-emf/EMFStandards/who0102/Worldmap5.htm . Last accessed: February $15^{\text {th }}$ 2015.

[12] I. Palatnik-de-Sousa, E. Costa Monteiro. "Transcranial Magnetic Stimulation: Terminology and Safety Aspects Concerning Therapeutic Protocol and Device Reliability". XXIV Congresso Brasileiro de Engenharia Biomédica, pp. 1325-1328, Uberlândia, Brazil, Oct. 2014

[13] Food And Drug Administration. Guidance for Industry and Food and Drug Administration Staff. Class II Special Controls Guidance Document: Repetitive Transcranial Magnetic Stimulation (rTMS) Systems. 2011.

[14] A. V. Peterchev, T. A. Wagner, P. C. Miranda, M. A. Nitsche, W. Paulus, S. H. Lisanby, A. Pascual-Leone, M. Bikson, Fundamentals of Transcranial Electric and Magnetic Stimulation Dose: Definition, Selection and Reporting Practices. Brain Stimulation. 2012; 5 (4), 435-453.

[15] International Electrotechnical Comission International Standard. Medical Electrical Equipment - Part 1: General requirements for basic safety and essential performance. 2005

[16] Council Directive 93/42/EEC of 14 June 1993 concerning medical devices. Official Journal of the European Union

[17] The International Commission on Non-Ionizing Radiation Protection, Guidelines for limiting exposure to time varying electric and magnetic fields $(1 \mathrm{~Hz}-$ 100 kHz). vol. 99 (6), 818-836. Health Physics. 2010.

[18] The International Commission on Non-Ionizing Radiation Protection, Guidelines for limiting exposure to electric fields induced by movement of the human body in a static magnetic field and by time-varying magnetic fields below $1 \mathrm{~Hz}$. Health Physics. vol. 106 (3), 418-425. 2014.

[19] The International Commission on Non-Ionizing Radiation Protection, Guidance on determining compliance of exposure to pulsed fields and complex non-sinusoidal waveforms below $100 \mathrm{kHz}$ with ICNIRP guidelines. Health Physics. vol. 84 (3), 383387. 2003.

[20] Karlström EF, Lundström R, Stensson O, Mild KH. Therapeutic staff exposure to Magnetic Field Pulses During TMS/rTMS Treatments. Bioeletromagnetics. vol. 27: 156-158. 2006.

[21] Lu, Mai and Ueno, Shoogo. Dosimetry of typical transcranial magnetic stimulation devices, Journal of Applied Physics, vol. 107, 2010.

[22] Bureau International de Poids et Mesures. International Vocabulary of Metrology - Basic and general concepts and associated terms (VIM). $3^{\text {rd }}$ edition. 2012.

[23] Bureau International de Poids et Mesures. Evaluation of measurement data - Guide to the expression of uncertainty In 2008. 


\title{
XXIV Congresso Brasileiro de Engenharia Biomédica
}

Transcranial Magnetic Stimulation: Terminology and safety aspects concerning therapeutic protocol and device reliability

\author{
Palatnik-de-Sousa, Iam*, Costa Monteiro, Elisabeth* \\ * Postgraduate Program in Metrology, Pontifical Catholic University of Rio de Janeiro, Rio \\ de Janeiro, Brazil
}

email: iam.palat@gmail.com

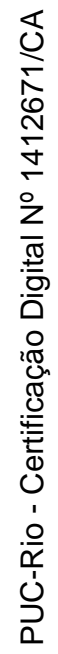


Abstract: An evaluation of Transcranial Magnetic Stimulation (TMS) safety parameters issues concerning the current situation of therapeutic protocol and device reliability is presented. Harmonization in terminology was analyzed, as well as possible misunderstandings that might arise from the lack of it. A notable example studied is the recommendation of safety parameters by the 2012 resolution of the Brazilian Federal Council of Medicine, when compared to international consensus and guidelines. The TMS device reliability is discussed considering technical standards and the International Commission on Non-Ionizing Radiation Protection reference limits for magnetic field exposure, applicable to both patients and of TMS systems.

Keywords: Transcranial Magnetic Stimulation, Metrological Reliability, Safety, Terminology, Stimulation Protocol.

\section{Introduction}

Transcranial Magnetic Stimulation (TMS) is a technique in which a rapidly changing current is passed through a small coil which is placed on the scalp [1]. The magnetic field generated by the coil allows getting electric energy across the scalp and skull without the pain of direct percutaneous electrical stimulation.

The TMS pulses can depolarize neurons and, when repeated pulses are applied, they can modulate cortical excitability, depending on the parameters of stimulation. This has behavioral consequences and therapeutic potential [2].

In the early stages of TMS, efforts were employed in the analysis of this technique as a tool for neural imaging, but as researches developed, new modalities and applications were described. This resulted in a growing lexicon regarding this field of study [1]. Notably, repetitive Transcranial Magnetic Stimulation (rTMS), consisting in performing TMS with particular time varying patterns, was shown to be an invaluable asset in the treatment of medication resistant depression, among other neurologic and psychiatric disorders.

Besides the clinical efficacy of TMS and all its modalities, another relevant research subject that has been approached by several studies concerns the safety parameters of stimulation protocols $[1,2]$. There were two major international workshops on safety of TMS and ethical aspects. These were held in 1996 and 2008, and the relevant generated information was compiled into tables of recommended values $[1,2]$.

Another important safety aspect that has to be taken into account concerning patients and therapeutic staff is the magnetic field exposure limits according to reference levels recommended by the International Commission on Non-Ionizing Radiation Protection (ICNIRP) [3, 4, 5].

Regarding the device reliability [6], TMS is currently approved by Health Surveillance Agencies of Israel, Canada, New Zealand, Brazil, Australia, United States, European Union, among others [2, 7, 8]. In Brazil, although already approved for use by the Brazilian National Health Surveillance Agency (Agência Nacional de Vigilância Sanitária - ANVISA) since 2007, TMS was recognized as a scientifically valid clinical procedure for utilization in health care on medical practice with the resolution 1.986/2012, published by the Federal Council of Medicine (Conselho Federal de Medicina - CFM) [8]. This acknowledgement regarded applications of surface TMS for the treatment of uni and bipolar depressions, hearing hallucinations on schizophrenias and neurosurgery planning. Notably, treatment parameters were recommended for each of those health disorders. In the 
mentioned CFM resolution, surface TMS for other indications, as well as deep TMS, remained being considered as experimental procedure.

Considering that full and reliable reports of the parameters that characterize TMS stimulation protocols are especially relevant for the advancement of research as well as their clinical effectiveness, it is fundamental to harmonize the employed terminology. The uniformity of dose description contributes to reproducibility, comparability, accurate interpretation, accomplishment of the desired clinical outcome, and prevention of adverse events.

Besides the complete and proper stimulus description, aspects regarding device reliability, including safety and performance checks should be satisfactorily considered [6]. To be registered by health agencies, TMS devices must demonstrate compliance with several technical standards. However, up to the present, no specific standard for TMS devices was published.

The present work envisioned bringing forth some of the details and possible issues that arise from the safety concerns encompassing terminology of stimulation protocols and TMS device reliability. For this purpose, among other studies concerning TMS safety, publications reporting consensus for safe treatment parameters, relevant safety guidelines published by regional and international institutions and any applicable technical standards pertaining to this issue were evaluated.

\section{Harmonization of TMS stimulation dose} parameters report

In Brazil, the treatment parameters for TMS currently recommended by the CFM consist on the frequency and intensity of the stimulus, the duration of trains, the number of trains, the total number of pulses, the interval between trains, the number of days of treatment and the location to apply the stimulus [8].
Notably, the total number of pulses is presented on the CFM document with a dot separating the first two digits from the three last ones. This notation does not follow the International System of Units (SI) [9] and can generate ambiguity. For instance, the indication of "25.000" as the total number of pulses for a depression treatment using a $10 \mathrm{~Hz}$ frequency could be interpreted as 25 or 25000 . One may feel compelled, however, to assume the latter value, since the total number of pulses is an integer number and there would be no need to present it with three decimal digits.

The CFM's recommendations [8] were then compared to the Consensus Statement published by Rossi et al [2] and to a recently published discussion regarding magnetic stimulation dose [10] (Table 1). This comparison pointed out non-uniformities in terminology and in the set of parameters considered for the protocol report.

Table 1: Comparison between the CFM [8] and the international consensus recommendations [2].

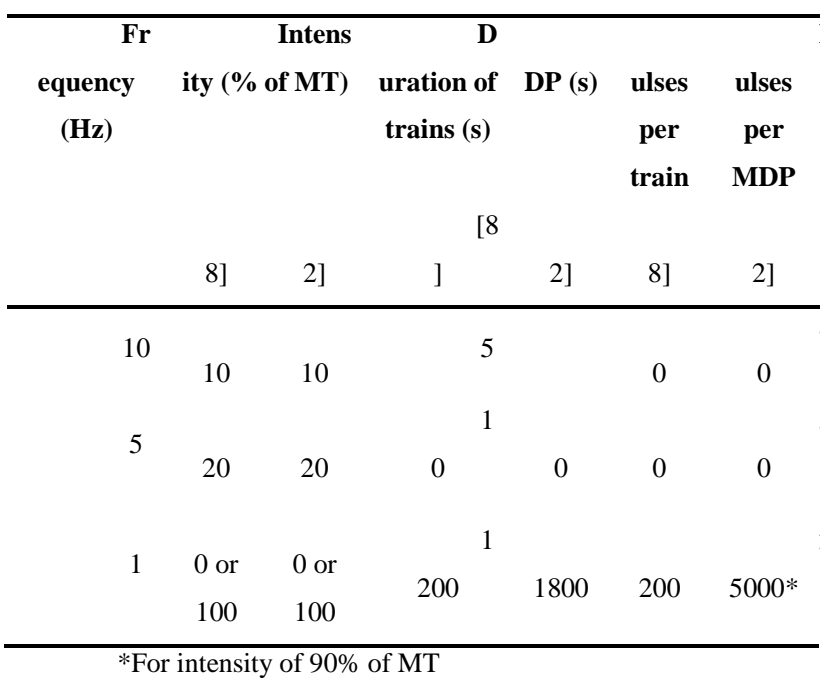

The international meeting consensus mentions that an inter-train interval of $5000 \mathrm{~ms}$ is considered safe for stimulation frequencies of less than $20 \mathrm{~Hz}$ with groups of 10 trains [2]. Although the CFM recommends a 
higher number of trains it also allows for a much higher inter-train interval [8]. It then says that the maximum duration of pulses (MDP) for individual TMS trains should not exceed the values provided on the table. Since one individual train corresponds to a group of pulses, the MDP would be readily comparable to the duration of trains shown in the CFM recommendations [8]. Indeed, these values agree, as can be seen in Table 1 , in which CFM values for the pulses in one train are found by multiplying the frequency by the duration of the train. Values related to the maximum duration of pulses taken from the international consensus [2]. Numbers preceded by the signal ">" are the longest values tested up to the point of the consensus meeting.

Since there were no indications for stimulations at $80 \%$ of motor threshold (MT), the closest comparable value of $90 \%$ was used in table 1 , as it basically coincides with the values for $100 \%$.The calculations also indicate that "Total number of pulses" in the CFM's document refers to the entire treatment, and not to each individual session. While this is in agreement with the recently published reporting suggestions for magnetic stimulation dose [10], it also means that the total number of pulses is not a fixed value for all cases and should be recalculated whenever the number of treatment days was decided to be different from 20. It could be useful to stress that the quantity refers to the whole duration of the treatment.

The terminology used for the described parameters in the Brazilian CFM document is closer to the suggestions recently published in the literature [10] than to terms such as the MDP parameter considered in the 2008 international meeting consensus [2,9]. While this points towards a harmonization in terminology, there are several other relevant parameters for reporting and reproducing research and clinical protocols that aren't currently considered by CFM document to be informed. These include stimulus waveform related parameters such as a complete characterization of the coil current waveform encompassing pulse shape, amplitude, width and polarity as well as coil configuration related parameters such as the winding shape, diameter, number of turns in each winding, core dimensions and material and any parameters of auxiliary coils or windings [10].

Uniformity in reporting these stimulation parameters is paramount to ensure sufficient information so that doses can be reproducible, for both research and clinical purposes.

\section{Reliability of TMS devices}

Medical devices must undergo tests of compliance to relevant standards containing general and specific safety and performance requirements promulgated by the International Electrotechnical Comission (IEC) and others that apply, in order to be registered by Health Surveillance Agencies [6].

The most relevant standards that TMS devices must comply with include the IEC 60601-1, IEC 60601-1-2, IEC 60601-1-4, and, since, up to the present there is no specific standard for TMS, the choice is to meet the specific parameters of the IEC 60601-2-10 standard, which contains specific requirements for the safety and performance of nerve and muscle stimulators.

However, the IEC 60601-2-10 standard states that, among others, equipment destined for brain stimulation and for neurological research should specifically be excluded from that standard's fields of application. Namely, TMS devices fall under this category.

This denotes the lack of a specific standard for brain stimulation equipments, most notably for TMS ones, since the operation principles of magnetic stimulations that induce currents on nerves and those that directly deliver currents via electrodes are quite different and require different safety prescriptions. 
For instance, taking into account the magnetic field exposure reference limits published by ICNIRP [3, 4, 5], specific requirements arise to be considered for TMS devices safety. Hazards regarding staff exposure to doses that surpass the recommended values by ICNIRP at distances of about less than $70 \mathrm{~cm}$ from the coil surface are pointed out by Kälstrom et al [11].

Much more restrictive is the fact that the ICNIRP reference levels were determined for exposure conditions in which the variation of electromagnetic fields over the body is small [4]. It is further stated that often the field source is close to the body making the field non-uniform or very localized, and that standardization bodies have the task to give further guidance on the specific exposure situations in which space averaging over the body can be applied, as well as deriving new reference levels for special types of nonuniform exposures. It is likely that TMS falls into one of these cases, since the fields generated by the stimulation coils are notably not distributed uniformly in space.

\section{The ICNIRP guideline mentions that all scientific} data and their interpretation are subject to some degree of uncertainty, and that this is compensated for by

is insufficient information about all the possible sources of uncertainty to provide a rigorous basis for establishing reduction factors over the whole frequency range and for all modulation patterns [4]. The degree of caution to be applied when considering reduction factors for the available database would be, thus, a matter of expert judgment to a large extent.

In the recently published Directive 2013/35/EU [12], the European Comission undertakes the task of making available before July, 2016, non-binding practical guides that cover, among others, the aspects of spatial averaging of electric and magnetic fields, guidance for dealing with uncertainties in measurements and calculations and most notably guidance on demonstrating compliance in special types of nonuniform exposure in specific situations.

Lastly, quantities and unit symbols used in some user manuals for TMS systems do not follow the SI recommendations [9]. The use of the obsolete term "magnetic induction" for the quantity magnetic flux density and the use of the symbol $\mathrm{Tl}$ for the unit tesla, instead of $\mathrm{T}$, are some examples of non-conformities of manuals' terminology with the SI.

In order to guarantee the metrological reliability of TMS devices, a particular standard establishing specific criteria, in agreement with ICNIRP restrictions and SI terminology, and including the requisite of measurement uncertainty evaluation, should be elaborated.

\section{Discussion}

Given the described scenario, what seems like the most direct way of facilitating harmonization would be to use the same values, terminology and reporting framework of consensus reports and publications alike for the development of safety protocols, clinical and research reports, and other relevant documents $[2,10]$. It could be also important to express the protocol limiting values as a function of parameters that can be directly adjusted on the devices, to avoid the need of further complicating calculations.

In particular for the CFM resolution, it would be valuable to reassess the used terminology to express safety parameters and the set of information required to be provided for dose protocol framework, in agreement with the international consensus and recent discussions in literature [2, 10]. For sufficiency of information, some other parameters concerning coil configuration and stimulus waveform should be included in the CFM document. 
The absence of specific standards regarding TMS machines raises issues in ensuring different aspects of safety already discussed, and could hinder the reliability of the technique and its capability to assure the success of the therapeutic protocol.

Considering the current status of safety guidelines regarding exposure, it could also be argued that preliminary actions to better protect staff should be taken until proper dosage measurement guidelines for non uniformly distributed fields are published. In addition, research on the calculation and measurement of magnetic stimulation doses, as well as staff exposure, should be carried out to provide more data and allow for specific safety requirements definitions for a possible future particular standard for TMS. The inclusion of requirements of expression of measurement uncertainty among the criteria for conformity assessment of TMS devices is a crucial aspect in guaranteeing the proper dose delivering. Different physiological effects are produced by minor variations in the combinations of stimulation parameters, altering clinical outcomes.

\section{Conclusion}

This work analyzed and discussed relevant safety aspects concerning terminology of TMS protocols and the metrological device reliability.

The current status of TMS safety stimulation protocol presents non uniformities regarding terminology and set of parameters reported. In particular, comparing aspects of protocol reporting in the recently published Brazilian resolution of CFM with international literature and consensus indicated issues concerning terminology and lacking of relevant parameters for reporting clinical protocols that could allow for ambiguities.

Considering the lack of a particular standard directed specifically at TMS devices, and the inadequacy of the standard used to substitute it, its metrological reliability is a demand for a future publication of a particular standard establishing specific criteria for TMS, in agreement with ICNIRP restrictions and SI terminology, and including the requisite of measurement uncertainty evaluation.

Moreover, some form of precaution should also be considered for the TMS staff while the updated guidelines for the proper dosimetry studies are not published.

\section{Acknowledgements}

We thank the Brazilian funding agency $\mathrm{CNPq}$ for financial support.

\section{References}

[1] Wasserman EM, Risk and Safety of repetitive transcranial magnetic stimulation: report and suggested guidelines from the international Workshop on the Safety of Repetitive Transcranial Magnetic Stimulation, June 5-7, 1996. Electroencephalography and clinical Neurophysiology. 1998; 108, 1-16.

[2] Rossi S, Hallet M, Rossini PM, Pascual-Leone A, The Safety of TMS Consensus Group, Safety, ethical considerations, and application guidelines for the use of transcranial magnetic stimulation in clinical practice and research. Clinical Neurophisiology. 2009; 120 (12), 2008-2039.

[3] The International Commission on Non-Ionizing Radiation Protection, Guidance on determining compliance of exposure to pulsed fields and complex non-sinusoidal waveforms below $100 \mathrm{kHz}$ with ICNIRP guidelines. Health Physics. 2003; 84 (3), 383-387.

[4] The International Commission on Non-Ionizing Radiation Protection, Guidelines for limiting 
13 a 17 de outubro de 2014 - Center Convention, Uberlândia, MG, Brasil

exposure to time varying electric and magnetic fields $(1 \mathrm{~Hz}-100 \mathrm{kHz})$. Health Physics. 2010; 99 (6), 818836.

[5] The International Commission on Non-Ionizing Radiation Protection, Guidelines for limiting exposure to electric fields induced by movement of the human body in a static magnetic field and by time-varying magnetic fields below $1 \mathrm{~Hz}$. Health Physics. 2014; 106 (3), 418-425.

[6] Costa Monteiro E, Biometrologia: confiabilidade nas biomedições e repercussões éticas. Metrologia e Instrumentação. 2007; 6, 6-12.

[7] López-Ibor JJ, López-Ibor IL, Pastrana JI, Transcranial Magnetic Stimulation. Current Opinion in Psychiatry. 2008; 21, 1-5.

[8] Conselho Federal de Medicina, Resolução CFM $1.986 / 2012$.

[9] Bureau international des poids et measures. The International System of Units (SI). $8^{\text {th }}$ ed. Paris; 2006.

[10] Peterchev AV, Wagner TA, Miranda PC, Nitsche MA, Paulus W, Lisanby SH, Pascual-Leone A, Bikson M, Fundamentals of Transcranial Electric and Magnetic Stimulation Dose: Definition, Selection and Reporting Practices. Brain Stimulation. 2012; 5 (4), 435-453.

[11] Kalström EF, Lundström R, Stensson O, Mild KH. Therapeutic staff exposure to Magnetic Field Pulses During TMS/rTMS

Treatments. Bioeletromagnetics. 2006; 27: 156-158.

[12] Directive 2013/35/EU of the European Parliament and of the council of 26 june 2013 on the minimum health and safety requirements regarding the exposure of workers to the risks arising from physical agents (electromagnetic fields) $\left(20^{\text {th }}\right.$ individual Directive within the meaning of Article 16(1) of Directive 89/391/EEC) and repealing Directive
2004/40/EC. Official Journal of the European Union. 2013 